\title{
Diffraktive Phasenelemente für partiell-kohärente UV-Laserstrahlung
}

\author{
Dissertation \\ zur Erlangung des Doktorgrades \\ der Mathematisch-Naturwissenschaftlichen Fakultäten \\ der Georg-August-Universität zu Göttingen
}

vorgelegt von

Dirk Schäfer

aus Bremen

Göttingen 2001 
D7

Referent:

Prof. Dr. W. Lauterborn

Korreferent:

Prof. Dr. G. Marowsky

Tag der mündlichen Prüfung:

26.6.2001 


\section{Inhaltsverzeichnis}

$\begin{array}{ll}\text { Einleitung } & 1\end{array}$

1 Grundlagen $\quad 4$

1.1 Paraxiale Beugungsintegrale . . . . . . . . . . . . . . . . 4

1.2 Abtastheorem . . . . . . . . . . . . . . . . . 5

1.3 Diffraktive Phasen- und Amplitudenelemente . . . . . . . . . . . . . . 6

1.4 Iterativer Fourier Transformationsalgorithmus (IFTA) . . . . . . . . . . 8

1.4.1 Der Basis IFT-Algorithmus . . . . . . . . . . . . . . 8

1.4.2 Quantisierung und Fresneltransformation (FRT) . . . . . . . . . 10

1.5 Der verwendete Excimer-Laser . . . . . . . . . . . . . . . . . . 12

1.6 Homogenisierung durch Apertursegmentierung . . . . . . . . . . . . . . 13

1.7 Festlegung grundlegender Parameter der DPE und ihre Herstellung . . . . 14

2 Modellierung partiell kohärenter Strahlung $\quad 17$

2.1 Das Strahlungsfeld als stochastischer Prozess . . . . . . . . . . . . . . 17

2.2 Die Kohärenzfunktion $j \ldots \ldots \ldots \ldots$

2.3 Intensität im Fernfeld und Betrag der Kohärenzfunktion $j$. . . . . . . . . 19

2.4 Hauptstrahlrichtungen im Nahfeld . . . . . . . . . . . . . . . . . . . . 22

3 Strahlformung und Homogenisierung mit DPE 24

3.1 Prinzip des optischen Systems . . . . . . . . . . . . . . . . 24

3.2 Design und Berechnung des DPE Nr. I . . . . . . . . . . . . . 25

3.3 Experiment . . . . . . . . . . . . . . . . . . 27

3.3.1 Messung der Stufentiefen des DPE Nr. I . . . . . . . . . . . . . 27

3.3.2 Optische Rekonstruktion des DPE Nr. I mit HeNe-Laser . . . . . . 28

3.3.3 Optische Rekonstruktion des DPE Nr. I mit KrF Excimer-Laser . . 29

3.3.4 Effizienzmessung von DPE Nr. I . . . . . . . . . . . . . . . . . 33

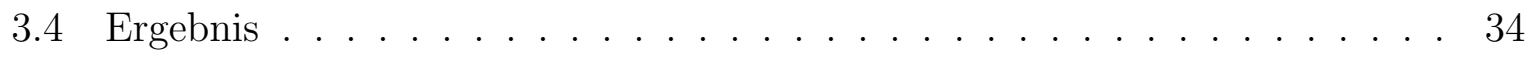

4 Korrektur der divergenten Wellenfrontcharakteristik $\quad 36$

4.1 Design und Berechnung der DPE Nr. II/III . . . . . . . . . . . . . . 36

4.2 Experiment . . . . . . . . . . . . . . . . . . . . . . . 39

4.2.1 Messung der Stufentiefen der DPE Nr. II/III . . . . . . . . . . . . . 39

4.2.2 Optische Rekonstruktion der DPE Nr. II/III mit KrF Excimer-Laser 41

4.2 .3 Effizienzmessung von DPE Nr. II/III . . . . . . . . . . . . . . . . . 44 
4.3 Simulation der Beugungseffizienz in Abhängigkeit von Ätzfehlern für DPE Nr. II . . . . . . . . . . . . . . . . . . . . . . . . . 45

4.4 Strukturfehler bei DPE Nr. II . . . . . . . . . . . . . . . . . . . 47

4.5 Ergebnis . . . . . . . . . . . . . . . . . . . . . 49

5 Korrektur der ortsaufgelösten Kohärenzeigenschaften 50

5.1 Ortsaufgelöste Fernfeldmessungen . . . . . . . . . . . . . . . . 50

5.1.1 Ortsaufgelöste Fernfeldmessung des L1000-Strahls mit Prismenkompressor . . . . . . . . . . . . . . . 51

5.1.2 Ortsaufgelöste Fernfeldmessung des L1000-Rohstrahls . . . . . . . . 51

5.2 Numerische Deconvolution . . . . . . . . . . . . . . . . . 54

5.2 .1 Angewendete Lösungsverfahren . . . . . . . . . . . . . . . . 55

Gauss-Elimination und Matrix-Inverse. . . . . . . . . . . . . 55

FFT-Deconvolution. . . . . . . . . . . . . 55

Iterative Deconvolution. . . . . . . . . . . . . 56

Deconvolution durch Singulärwertzerlegung. . . . . . . . . . . 56

5.2.2 Singulärwertzerlegung und Matrixdarstellung der Convolution . . . 56

Singulärwertzerlegung (SVD). . . . . . . . . . . . 56

Toeplitz Matrizen und Faltung. . . . . . . . . . . . . 57

Zweidimensionale, separierbare Faltungskerne und ihre SVD. . . . . 59

5.2.3 Deconvolution durch Abbruch-Regularisierung (TSVD) mit dem

Fernfeld $I_{\mathrm{f}}$ des L1000 mit Prismenkompressor. . . . . . . . . . . . . 61

5.2.4 Exkurs: TSVD-Deconvolution mit dem Fernfeld des L1000 Rohstrahls 65

5.2.5 Deconvolution durch Tikhonov-Regularisierung. . . . . . . . . . . 68

5.3 Design und Berechnung des DPE Nr. IV . . . . . . . . . . . . . . 69

5.4 Experiment . . . . . . . . . . . . . . . . . . 71

5.4.1 Charakterisierung des zur Rekonstruktion von

DPE Nr. IV verwendeten KrF Excimer-Laserstrahls . . . . . . . . . 71

5.4.2 Optische Rekonstruktion des DPE Nr. IV

mit KrF Excimer-Laser . . . . . . . . . . . . . . . . . . . . 74

5.4.3 Effizienzmessung von DPE Nr. IV . . . . . . . . . . . . . . . . 75

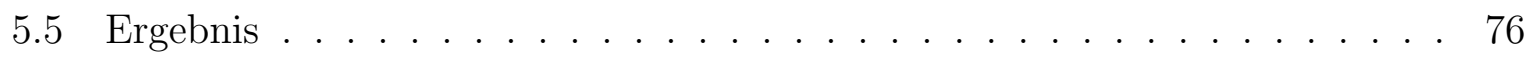

$\begin{array}{ll}\text { Zusammenfassung } & 77\end{array}$

$\begin{array}{ll}\text { A Konventionen und Definitionen } & 79\end{array}$

$\begin{array}{ll}\text { Abbildungsverzeichnis } & 81\end{array}$

$\begin{array}{lr}\text { Tabellenverzeichnis } & 83\end{array}$

$\begin{array}{lr}\text { Literaturverzeichnis } & 84\end{array}$ 


\section{Einleitung}

Die Diffraktive Optik beschäftigt sich mit optischen Komponenten, die durch eine beugende Struktur ein Strahlungsfeld gezielt modifizieren. Der Wegbereiter für solche Diffraktiven Optischen Elemente (DOE), die komplexe Veränderungen der Amplitude und Phase eines kohärenten Strahlungsfeldes vornehmen können, war die auf Gabor [19] zurückgehende Holographie. Ein Hologramm zeichnet die Interferenz einer Referenzwelle mit der an einem realen Objekt gebeugten Welle als Intensitätsmodulation auf. Bei Beleuchtung des Hologramms mit der Referenzwelle wird neben der verbreiterten nullten Ordnung das direkte und das konjugierte Bild des Objekts rekonstruiert. Durch den technologischen Fortschritt auf den Gebieten der Mikrostrukturierung und der digitalen Rechner können beugende Strukturen mit variabler Transmission berechnet und hergestellt werden, ohne dass ein reales Objekt zur Erzeugung eines solchen digitalen Hologramms benötigt wird. Im Übersichtsartikel von W.-H. Lee [38] sind Anwendungen für die optische Datenverarbeitung und das Testen von optischen Oberflächen mit digitalen Hologrammen beschrieben.

Um die Lichtverluste durch das beugende Element zu verringern, wurden transparente Elemente entwickelt, die durch ihre Oberflächenstruktur nur die Phase des einfallenden Wellenfeldes verändern und im Idealfall nur das Objekt rekonstruieren. Zur Erzeugung von Punktmustern präsentierten Lesem et al. [39] im Jahre 1969 erstmals eine solche mit dem Computer berechnete Phasenverteilung, die in gebleichte Photoemulsion übertragen wurde, und nannten dieses Element „Kinoform“. Kinoforme werden heute meist als Diffraktive Phasenelemente (DPE) bezeichnet. Eine frühe Anwendung war die gleichzeitige Erzeugung von Kavitationsblasen durch die verschiedenen Foki eines mit einem DPE geformten Rubin-Laserstrahls [26].

Die Diffraktive Optik ist mittlerweile zu einem stark expandierenden Zweig der modernen Optik mit vielfältigen Anwendungsgebieten [50] geworden. Ein wichtiges Teilgebiet ist die Formung von Laserstahlung in vorgegebene Geometrien und Intensitätsprofile sowie die Teilung eines Strahls in mehrere Einzelstrahlen mit definierten relativen Intensitäten.

Effiziente Strahlformung ist insbesondere wichtig für kurzwellige UV-Strahlung von Excimer-Lasern, die umfangreiche Anwendung in der Mikromaterialbearbeitung finden [4]. Für viele dieser Anwendungen sind hohe Energiedichten und somit eine möglichst effektive Ausnutzung der Strahlung erforderlich. Die inhomogene Intensitätsverteilung, die hohe Divergenz und die geringe Fokussierbarkeit (geringe räumliche Kohärenz) der Excimer-Laserstrahlung muß den jeweiligen Anforderungen angepaßt werden. Die Erzeugung von homogenen Profilen mit rechteckigen Strahlquerschnitten kann mit Multifacettenelementen [14] refraktiv [41] oder diffraktiv [43] realisiert werden. Die Formung 
von Excimer-Laserstrahlung mit Diffraktiven Phasenelementen zur effizienten Mikromaterialbearbeitung konnte erfolgreich für ringförmige Foki [5], [52], Spot-Arrays [27] oder Sechsecke [52] demonstriert werden. Das Ergebnis der Strahlformung hängt dabei von den Kohärenzeigenschaften der verwendeten Laserstrahlung ab. Henning und Scholl [25] untersuchten die Auswirkung partieller Kohärenz auf die Formung rechteckiger Strahlquerschnitte mit segmentierten Mikro-Spiegeln und optimierten das Ergebnis durch Anpassung der Fresnel-Zahl des optischen Aufbaus an den Kohärenzgrad der Strahlung. Turunen et al. [49] stellten ein periodisches DPE her, das durch Überlagerung verschiedener Beugungsordnungen ein homogenes Profil mit rechteckigem Strahlquerschnitt erzeugt. Beyerlein et al. [7] simulierten die Rekonstruktion von DPE bei partiell-kohärenter Beleuchtung basierend auf Intensitätsmessungen im Fernfeld eines Excimer-Laserstrahls. Für das Design eines Multifacettenelements diente die daraus berechnete Kohärenzlänge der Strahlung als untere Grenze für die Aperturgröße einer einzelnen Facette (vgl. auch Abschnitte 1.5,1.6).

Alle oben genannten Arbeiten benutzen zum Design der Phasenfunktion der DPE entweder strahlenoptische Methoden wie geometrische Transformationen [11], [15], [16], oder lösen die paraxialen Beugungsintegrale direkt. Für komplizierte Strahlformungsgeometrien können diese Beugungsintegrale nicht direkt gelöst werden, oder die Lösung besteht aus einer variablen Phasen- und Amplitudenfuktion und kann somit nicht als DPE realisiert werden. Die Strahltransformation mit geometrischen Methoden ist limitiert, da nur Strahlprofile erzeugt werden können, deren Träger einfach-zusammenhängende Gebiete bilden. Zur Berechnung von Phasenelementen, die kohärente Laserstrahlung hingegen in annähernd beliebige Strahlgeometrien formen können, sind Iterative Fourier Transformations-Algorithmen (IFTA) entwickelt worden [56]. Die Übertragung dieser Technik auf partiell-kohärente Strahlung ist wiederum nur begrenzt möglich, da die Information der hohen Raumfrequenzen aufgrund der kurzen räumlichen Kohärenzlänge verloren geht. Dies führt bei der Erzeugung von Kastenprofilen zu unerwünschter Abrundung und Ausschmierung der Flanken. Zur Formung von partiell-kohärenter Strahlung sind daher IFTA-Designs bisher kaum verwendet worden. Vahimaa [51] präsentierte numerische Simulationen mit einem IFT-Algorithmus basierend auf der spektralen Kohärenzfunktion eines Schell-Modell-Strahls [40]. Bei der von Kreutz et al. [36] veröffentlichten Arbeit ist die benutzte Design-Methode der gezeigten komplexen Strahltransformation mit ExcimerLaserstrahlung nicht angegeben, vermutlich handelt es sich hierbei um ein IFTA-Design.

Ziel dieser Arbeit ist es, die Möglichkeiten Diffraktiver Phasenelemente zur Formung partiell-kohärenter Excimer-Laserstrahlung zu erweitern. Es wird die Strahlformung und Homogenisierung der Excimer-Laserstrahlung mit Multifacetten-DPE untersucht. Dabei liegt das Hauptaugenmerk auf der theoretischen Entwicklung neuer Design-Konzepte und deren experimenteller Realisierung und Überprüfung. Dies wird an Beispielen durchgeführt, die für die Materialbearbeitung mit Excimer-Lasern interessant sind. In Kapitel 1 werden das IFTA-Design und die grundlegenden Konzepte zur Strahlformung 
entwickelt sowie der verwendete Excimer-Laser vorgestellt. Die Modellierung partiellkohärenter Strahlung und deren Beugungstheorie behandelt Kapitel 2. Strahlformung und Homogenisierung mit einem DPE wird in Kapitel 3 demonstriert. Dabei erfolgt die Strahlformung innerhalb einer Facette (auch Subapertur genannt), deren Phasenfunktion mit einem herkömmlichen IFTA-Design berechnet wird. Die Homogenisierung entsteht durch die (inkohärente) Überlagerung der Signale aus den einzelnen Subaperturen. Diese Überlagerung wird im Kapitel 4 durch die Berücksichtigung der Wellenfront des ExcimerLaserstrahls verbessert. Die Möglichkeiten der Verbesserung der Strahlformung innerhalb der Subaperturen durch Berücksichtigung der ortsaufgelösten Kohärenzeigenschaften mit einem neuen, erweiterten IFTA-Design werden in Kapitel 5 untersucht. 


\section{Grundlagen}

\subsection{Paraxiale Beugungsintegrale}

Innerhalb der skalaren Beugungstheorie liefert die paraxiale Näherung des Kirchhoffschen Beugungsintegrals einen Ausdruck für die Ausbreitung eines zweidimensionalen optischen Signals. In einer Ebene senkrecht zur z-Achse sei das monofrequente Signal $E(u, v, z=0)$ mit der Wellenlänge $\lambda$ gegeben, in der Ebene $z=z_{0}$ erhält man in der Fresnel-Approximation folgenden Ausdruck für das Beugungsbild (vgl. z.B. [37]):

$$
\begin{aligned}
& E\left(x^{\prime}, y^{\prime}, z=z_{0}\right)=\frac{\exp \left(i \frac{2 \pi}{\lambda} z_{0}\right)}{i \lambda z_{0}} \exp \left[i \frac{\pi}{\lambda z_{0}}\left({x^{\prime}}^{2}+y^{\prime 2}\right)\right] \\
& \int_{-\infty}^{\infty} \int_{-\infty}^{\infty} E(u, v, z=0) \exp \left[i \frac{\pi}{\lambda z_{0}}\left(u^{2}+v^{2}\right)\right] \exp \left[-2 \pi i\left(\frac{x^{\prime}}{\lambda z_{0}} u+\frac{y^{\prime}}{\lambda z_{0}} v\right)\right] \mathrm{d} u \mathrm{~d} v .
\end{aligned}
$$

Für große Entfernungen $z_{0}$ von einer begrenzten Quelle bei $z=0$ kann der Phasenfaktor im Integral vernachlässigt werden und man erhält die Fraunhofer-Approximation:

$$
\begin{aligned}
& E\left(x^{\prime}, y^{\prime}, z_{0}\right)=\frac{\exp \left(i \frac{2 \pi}{\lambda} z_{0}\right)}{i \lambda z_{0}} \exp \left[i \frac{\pi}{\lambda z_{0}}\left(x^{\prime 2}+y^{\prime 2}\right)\right] \\
& \int_{-\infty}^{\infty} \int_{-\infty}^{\infty} E(u, v, 0) \exp \left[-2 \pi i\left(\frac{x^{\prime}}{\lambda z_{0}} u+\frac{y^{\prime}}{\lambda z_{0}} v\right)\right] \mathrm{d} u \mathrm{~d} v .
\end{aligned}
$$

Durch Einführen von Raumfrequenzen $x=x^{\prime} / \lambda z_{0}$ und $y=y^{\prime} / \lambda z_{0}$ sowie der Abkürzung

$$
F T[f(u, v)](x, y)=\int_{-\infty}^{\infty} \int_{-\infty}^{\infty} f(u, v) \exp [-2 \pi i(x u+y v)] \mathrm{d} u \mathrm{~d} v
$$

für die Fouriertransformation, läßt sich die Intensitätsverteilung $I=|E|^{2}$ kompakt schreiben als:

$$
\begin{aligned}
I(x, y) & =\frac{1}{\lambda^{2} z_{0}^{2}}|F T[E(u, v)]|^{2} \\
& \propto|F T[E(u, v)]|^{2}
\end{aligned}
$$

wobei $u, v$ Ortskoordinaten in der Ebene des beugenden Elements sind und $x, y$ Raumfrequenzen in der Fraunhofer-Ebene. 


\subsection{Abtasttheorem}

Die benötigten Definitionen und Sätze werden in der später verwendeten zweidimensionalen Form in kartesischen Koordinaten angegeben. Für eine allgemeinere Darstellung siehe beispielsweise Kapitel 6 in [42].

\section{Bandbegrenzte Funktionen:}

Eine Funktion $f(\mathbf{x})=f(x, y)$ heißt „bandbegrenzt“(„,bandlimited“), wenn für ihr Spektrum $F(\mathbf{u})=F(u, v)=F T[f(\mathbf{x})]$ gilt:

$$
F(\mathbf{u})=F(\mathbf{u}) \operatorname{rect}(\mathbf{u}, \Delta \mathbf{u}),
$$

d. h. ihr Spektrum $F(\mathbf{u})$ ist null für $|u|>\Delta u / 2$ und $|v|>\Delta v / 2$. Der Parameter $\Delta \mathbf{u}=(\Delta u, \Delta v)$ heißt Bandbreite von $f$.

\section{Abtasttheorem (Sampling-Theorem):}

Ist die Funktion $f(\mathbf{x})=f(x, y)$ bandbegrenzt, läßt sie sich ausdrücken durch:

$$
\begin{aligned}
f(\mathbf{x}) & =\sum_{\mathbf{n} \in Z^{2}} f(\underline{\underline{Q}} \mathbf{n}) \operatorname{sinc}(\underline{\underline{Q}} \mathbf{n}, \delta \mathbf{x}) \\
& =(f(\tilde{\mathbf{x}}) \operatorname{comb}(\tilde{\mathbf{x}}, \delta \mathbf{x})) * \operatorname{sinc}(\tilde{\mathbf{x}}, \delta \mathbf{x})
\end{aligned}
$$

Die sogenannte Sampling-Matrix $\underline{\underline{Q}}$ ist im kartesischen Fall $\underline{\underline{Q}}=\operatorname{diag}(\delta x, \delta y)$. Für den größtmöglichen Samplingabstand, das Nyquist-Sampling, gilt: $\delta x=$ $1 / \Delta u, \delta y=1 / \Delta v$.

Bandbegrenzte Funktionen sind glatt. Jede Abweichung von der Eigenschaft „glatt“ führt zu hohen Raumfrequenzen im Fourierspektrum und damit zum Verlust der Eigenschaft „bandbegrenzt“. Die Glattheit zwischen den Sample-Punkten schließt beliebige Variationen für bandbegrenzte Funktionen in diesen Zwischenbereichen aus.

Für die Strahlformung interessante Anwendungen sind aber gerade Rechteck-(Top-Hat-) Profile, die nicht bandbegrenzt sind. Wird für die Rekonstruktion einer solchen Funktion trotzdem gemäß dem Sampling-Theorem nur ein endlicher Bereich des Fourierspektrums verwendet, entstehen zwischen den Sampling-Punkten Abweichungen von der ursprünglichen Funktion. Diese Abweichungen heißen aliasing error [3]. Bei der optischen Rekonstruktion digital berechneter diffraktiver Elemente macht sich der aliasing error in ungewollten Intensitätsfluktuationen bemerkbar, die wegen ihrer unregelmäßigen Struktur auch „Speckles“ genannt werden. Dieser Begriff ist mit Vorsicht zu verwenden, da er in diesem Fall nicht durch Streuung an einer rauhen Oberfläche entstehende Speckles [37] bezeichnet. 


\subsection{Diffraktive Phasen- und Amplitudenelemente}

Ein diffraktives Element, das nur die Phase der einfallenden Welle verändert, heißt Kinoform [39] oder Diffraktives Phasenelement (DPE). Die Funktionswerte der Transmissionsfunktion $g \in \mathbb{C}$ eines DPE liegen alle auf dem Einheitskreis:

$$
g=\exp (i \psi), \quad|g|=1, \quad \psi \in \mathbb{R} .
$$

Anders ausgedrückt: Die Transmissionsfunktion $g$ ist eine reine Phasenfunktion. Soll in der Fraunhofer-Ebene ein optisches Signal endlicher Ausdehnung durch Beugung an einem DPE erzeugt werden, bedeutet dies gemäß der Definition aus Gleichung (1.4), dass die Phasenfunktion bandbegrenzt sein muss. Außer den linearen Phasenfunktionen, die die diffraktive Entsprechung der Prismen darstellen, gibt es jedoch keine bandbegrenzten Phasenfunktionen [53]. Also wird im allgemeinen Licht in unerwünschte Richtungen propagieren und die Beugungseffizienz geringer als 100\% sein. Als Beugungseffizienz wird das Verhältnis der Intensität in einem vorgegebenen Gebiet zur gesamten durch das diffraktive Element transmittierten Intensität bezeichnet. Als Systemeffizienz wird das Verhältnis der Intensität in dem vorgegebenen Gebiet zur gesamten auf das diffraktive Element eingestrahlten Intensität bezeichnet. Die Systemeffizienz berücksichtigt somit auch Verluste durch Reflexion und Absorption, die vom diffraktiven Element verursacht werden.

Ein diffraktives Element, das nur die Amplitude der einfallenden Welle verändert, heißt Diffraktives Amplitudenelement (DAE) [56]. Die Transmissionsfunktion ist gegeben durch:

$$
g \in[0,1] \in \mathbb{R}
$$

Diffraktive Phasenelemente haben demzufolge erheblich höhere Systemeffizienzen $\eta$ als DAE. Für den Fall binärer Phasen- und Amplitudenelemente kann ein quantitativer Wert angegben werden [56]:

$$
\eta_{D A E}=\pi^{-2} \eta_{D P E} \simeq \frac{1}{10} \eta_{D P E}
$$

Die gleichzeitige Kontrolle von Amplitude und Phase ist für die im Rahmen dieser Arbeit benutzten UV-Laser kaum möglich und immer mit einem zusätzlichen Verlust an Intensität verbunden. Aus diesem Grunde werden in dieser Arbeit ausschließlich Diffraktive Phasenelemente behandelt.

Bei der Herstellung von DPE müssen das Höhenprofil und die verwendete Wellenlänge aufeinander abgestimmt werden (vgl. Abb. (1.1)). Dieses kontinuierliche DPE muß die Wellenzüge an den Steilkanten phasengerecht wieder zusammenfügen. Die Höhe $h_{0}$ berechnet sich aus dem Quotienten der verwendeten Wellenlänge und der Differenz der Brechungsindices. Wird bei der Herstellung des DPE die optimale Höhe $h_{0}$ nicht erzeugt, nimmt die Intensität in der gewünschten Beugungsordnung $(m=1)$ ab, und Licht wird in andere Beugungsordnungen $(m=0,+2,-1,+3, \ldots$ ) abgelenkt. Die Abbildung (1.2) zeigt die Abhängigkeit der Intensität von Abweichungen $\Delta h$ von $h_{0}$ in einigen Beugungsordnungen für ein 1-dimensionales ideal geformtes DPE [17]. 


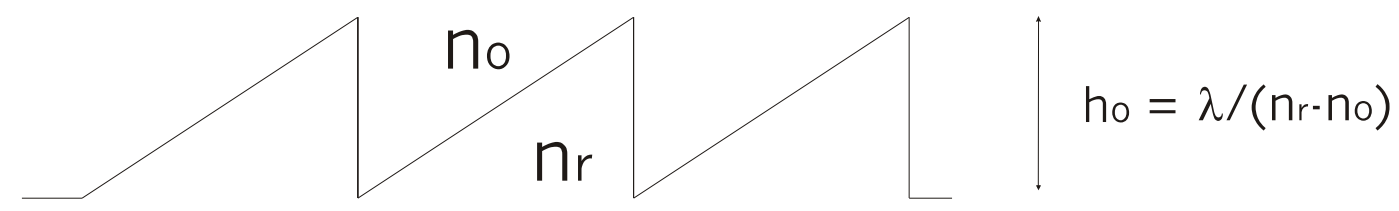

Abbildung 1.1: Kontinuierliches DPE

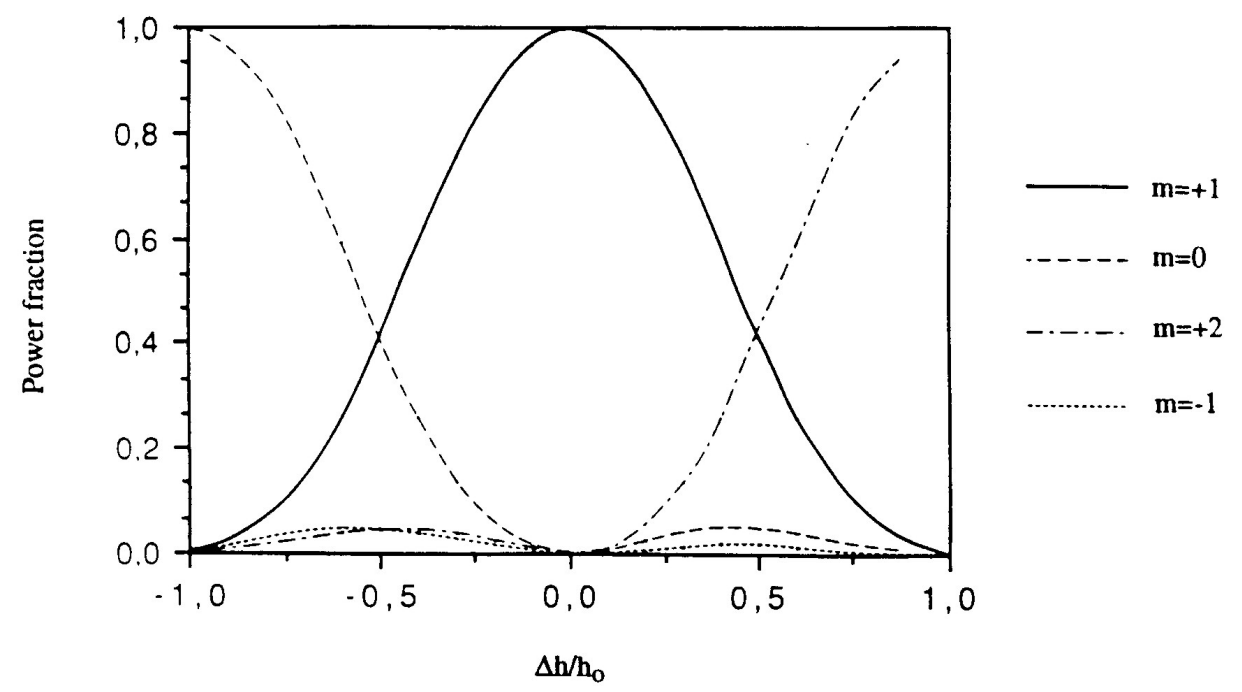

Abbildung 1.2: Auswirkung des Höhenfehlers $\Delta h$ auf die Beugungseffizienz (aus [17])

Bei quantisierten DPE wird nur eine diskrete Anzahl $Z$ von Höhenstufen realisiert. Das führt dazu, dass nicht der ganze Phasenhub von $2 \pi$ vorgenommen wird, sondern nur $(Z-1) / Z \cdot 2 \pi$. Damit folgt für die maximale Höhe $D$ eines quantisierten DPE (Abb. $(1.3))$ :

$$
D=(Z-1) / Z \cdot \lambda /\left(n_{r}-n_{0}\right)
$$

Durch die Approximation der kontinuierlichen Phasenverteilung durch $Z$ Stufen nimmt die Intensität $I$ in der gewünschten Ordnung $(m=1)$ ab. Für ein 1-dim DPE mit optimalen Stufenhöhen $D_{i}=D /(Z-1)$ gilt $[23]$ :

$$
I(m, Z)=(\operatorname{sinc}((m-1)+1 / Z))^{2} .
$$

Die sich daraus errechnende relative Intensität ist für einige ausgewählte Beugungsordnungen in Tabelle (1.1) angegeben. Es ist bisher noch nicht geglückt, einen ähnlichen Ausdruck für den zweidimensionalen Fall abzuleiten. Es gibt Arbeiten über obere Grenzen der Beugungseffizienz von DPE zur Rekonstruktion von komplexen Signalen [55], [33]. Falls nur die Intensität des Signals vorgegeben ist, kann die Phase des Signals als DesignParameter benutzt werden, um die Beugungseffizienz zu erhöhen. In diesem Fall können die eindimensionalen Ergebnisse aus (1.10) als Richtwerte angesehen werden. 


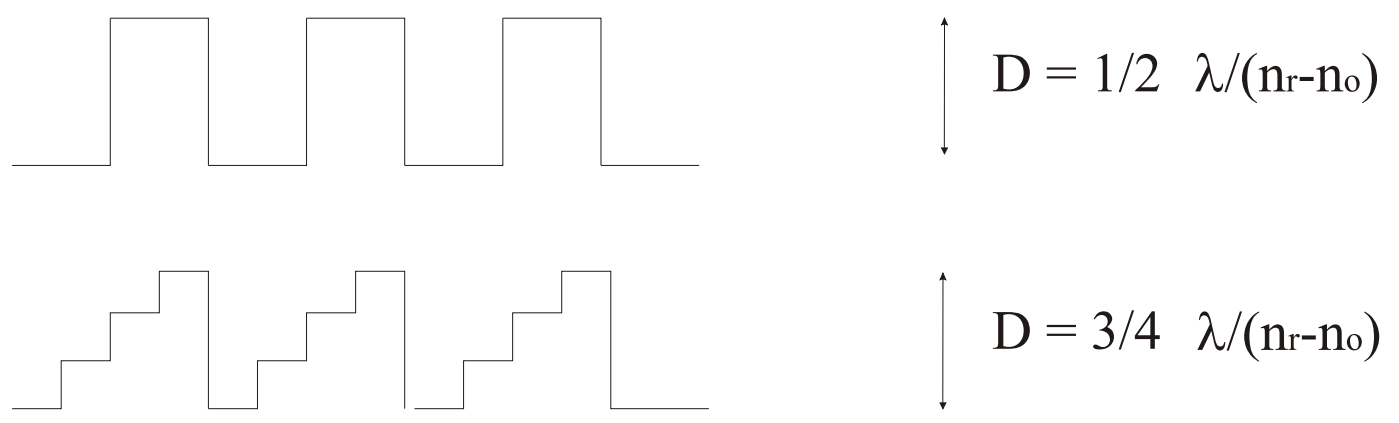

Abbildung 1.3: Quantisiertes DPE

\begin{tabular}{l|c|c|c|c} 
& $m=-1$ & $m=0$ & $m=+1$ & $m=+2$ \\
\hline$Z=2$ & 4.5 & 40.5 & 40.5 & 4.5 \\
$Z=3$ & 2.7 & 17.1 & 68.4 & 4.3 \\
$Z=4$ & 1.7 & 9.0 & 81.1 & 3.2 \\
$Z=8$ & 0.4 & 1.9 & 95.0 & 1.2
\end{tabular}

Tabelle 1.1: Beugungseffizienz (\%) in Abhängigkeit der Stufenanzahl eines DPE

\subsection{Iterativer Fourier Transformationsalgorithmus (IFTA)}

\subsubsection{Der Basis IFT-Algorithmus}

Soll Laserstrahlung geformt („beam shaping“) oder in verschiedene Teilstrahlen zerlegt werden („,beam splitting“), ist in der Regel die Kenntnis der komplexen Amplitude des Wellenfeldes in zwei Ebenen senkrecht zur Ausbreitungsrichtung ausreichend. Das beugende Element (DPE) in der DPE-Ebene verändert die als bekannt vorausgesetzte komplexe Amplitude der einfallende Strahlung, so dass in der Signal-Ebene in einem Abstand $z_{0}$ von der DPE-Ebene eine komplexe Amplitude entsteht, die dem vorgegebenen Signal $f^{0}$ möglichst nahe kommen sollte. Die Ausbreitung der komplexen Amplitude hinter dem DPE $g(u, v)$ wird durch einen Propagationsoperator $\mathcal{P}$ beschrieben. Die komplexe Amplitude $f(x, y)$ in der Signal-Ebene ergibt sich dann aus $f(x, y)=\mathcal{P}(g(u, v))$. Falls $\mathcal{P}$ invertierbar ist, kann die beugende Struktur $g$ prinzipiell durch $g=\mathcal{P}^{-1}\left(f^{0}\right)$ berechnet werden. Im einfachsten Fall ist $\mathcal{P}$ gegeben durch die Fouriertransformation $\mathcal{P}=F T$. Die inverse Fouriertransformation $g=F T^{-1}\left(f^{0}\right)$ eines beliebigen Signals $f^{0}$ wird im allgemeinen nicht zu einer Transmissionsfunktion $|t|=1$ für ein DPE gemäß Gleichung (1.7) 
führen. Wird die Lösung $g$ auf den Einheitskreis projiziert, so ergeben sich bei Propagation in die Signal-Ebene Abweichungen von der vorgegebnen Verteilung $f^{0}$. Bei einem Intensitätssignal $f^{0}=\left|f^{0}\right|$ können Abweichungen der Phase $\arg \left(f^{0}\right)$ toleriert werden. Die Amplitude muss wieder auf den Soll-Wert zurück gesetzt werden. Dieser Vorgang wird so oft wiederholt, bis die Abweichungen der Rekonstruktion die vorgegebene Fehlergrenze unterschreiten oder eine maximale Iterationszahl erreicht ist. Dies ist die zentrale Idee des in Abbildung (1.4) dargestellten Iterativen Fourier Transformationsalgorithmus (IFTA). Als Qualitätskriterien der Lösung $\psi$ dienen der Objekt-Fehler $r m s_{s}$ und die Effizienz $\eta$ (vgl. (A.3),(A.4)). Aus der umfangreichen Literatur zu diesem Algorithmus und seinen Abwandlungen sei nur auf den Überblicksartikel von Wyrowski und Bryngdahl [56] und einige ausgewählte Artikel verwiesen [30], [1], [10].

Signal Ebene

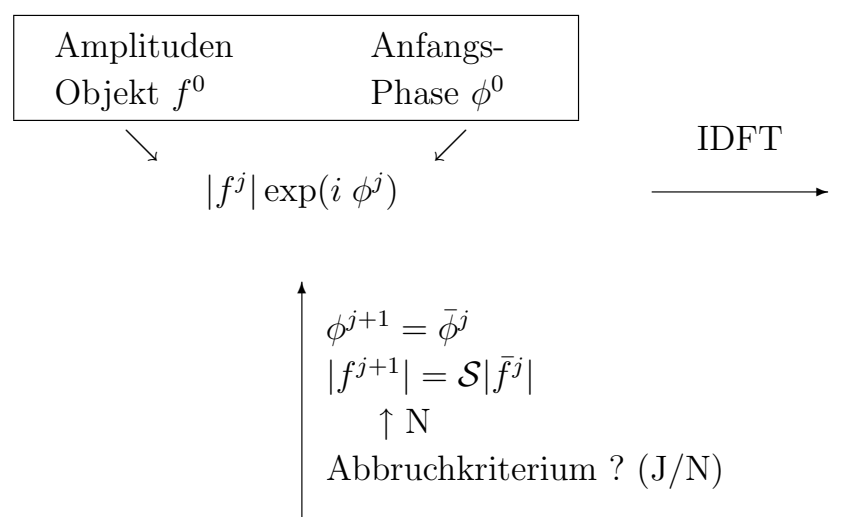

DFT

\section{$\underline{\text { DPE-Ebene }}$}

$$
\begin{aligned}
& \left|g^{j}\right| \exp \left(i \psi^{j}\right) \\
& \mid \begin{array}{l}
\bar{\psi}^{j}=\psi^{j} \\
\left|\bar{g}^{j}\right|=\mathcal{G}\left(\left|g^{j}\right|\right)
\end{array}
\end{aligned}
$$

$$
\left|\bar{g}^{j}\right| \exp \left(i \bar{\psi}^{j}\right)
$$

$\downarrow \mathrm{J}$

kontinuierliche Phasenfunktion $\psi$

Die Anfangsphase $\phi^{0}$ wird gewöhnlich als Zufallsverteilung von Zahlen aus dem Intervall $[0,2 \pi]$ gewählt. Das auf einem $N M$-Gitter diskretisierte Anfangsobjekt $f^{0}$ mit der Phase $\phi^{0}$ wird durch inverse diskrete Fouriertransformation (IDFT) in die DPE-Ebene zurückpropagiert. Die Amplitude $\left|g^{j}\right|$ in der DPE-Ebene wird auf den Wert des beleuchtenden Strahls gesetzt, für eine ebene Welle gilt:

$$
\left|\bar{g}^{j}\right|=\mathcal{G}\left(\left|g^{j}\right|\right)=c_{g} \quad \text { const }
$$

wobei $c_{g}$ so gewählt wird, dass das Parseval-Theorem der DFT erfüllt ist:

$$
\sum_{k, l=1}^{N, M}\left|\bar{g}_{k l}^{j}\right|^{2}=\frac{1}{N M} \sum_{k, l=1}^{N, M}\left|f_{k l}^{0}\right|^{2}
$$




\section{Grundlagen}

Die Division durch die Gesamtanzahl der Pixel $N M$ auf der rechten Seite folgt aus der unsymmetrischen DFT-Definition [48].

Die Phase $\bar{\psi}^{j}=\psi^{j}$ wird beibehalten und die neue DPE-Funktion in die Signal Ebene propagiert. Aus der resultierenden Amplitude $\left|\bar{f}^{j}\right|$ wird auf dem Träger von $\left|f^{0}\right|$ der skalierte Fehler $r m s_{s}\left(\left|f^{0}\right|,\left|\bar{f}^{j}\right|\right)$ berechnet. Ist dieser geringer als das vorgegebene Fehlerkriterium oder die maximale Iterationszahl erreicht, bricht der Algorithmus ab, und die kontinuierliche Phasenfunktion $\bar{\psi}^{j}$ definiert die Lösung. Ist keines der Abbruchkriterien erfüllt, wird die Phase $\phi^{j+1}=\bar{\phi}$ beibehalten und die Amplitude durch den Signaloperator $\mathcal{S}$ zurückgesetzt:

$$
\begin{aligned}
\left|f_{n m}^{j+1}\right|=\mathcal{S}\left|\bar{f}_{n m}^{j}\right|= & \left\{\begin{array}{c}
c_{s}\left|f_{n m}^{0}\right| \text { für } n, m \in \mathbb{I} \\
\left|\bar{f}_{n m}^{j}\right| \text { sonst, }
\end{array}\right. \\
& \text { wobei } \mathbb{I} \text { die Indexmenge des Trägers von } f^{0} \text { bezeichnet, } \\
& \text { und } c_{s} \text { gegeben ist durch [47]: } \\
c_{s}= & \frac{\sum_{(n, m) \in \mathbb{I}}\left|f^{0}{ }_{n m}\right|\left|\bar{f}_{n m}^{j}\right|}{\sum_{(n, m) \in \mathbb{I}}\left|f^{0}{ }_{n m}\right|^{2}}
\end{aligned}
$$

\subsubsection{Quantisierung und Fresneltransformation (FRT)}

Das Resultat des im vorigen Abschnitt dargestellten IFT-Algorithmus ist die Phasenfunktion $\psi$, die beliebige Werte im Intervall $[0,2 \pi]$ annimmt. Durch maskenbasierte lithographische oder ähnliche Herstellungsverfahren (vgl. Abschnitt (1.7)) können nur $Z$ diskrete Tiefenstufen in einem transparenten Material gefertigt werden. Mit einem Satz von $m$ Masken sind $Z=2^{m}$ verschiedene äquidistante Phasenlevel realisierbar [34]. Aus der Funktion $\psi$ wird nun eine quantisierte Version $\psi_{\mathrm{Q}}$ berechnet, die entsprechend nur $Z$ verschiedene Werte besitzt: $\psi_{\mathrm{Q}} \in\left\{0, \frac{1}{Z} 2 \pi, \ldots, \frac{Z-1}{Z} 2 \pi\right\}$. Die Projektion der $\psi$-Werte auf den nächstgelegenen Wert, der $Z$ möglichen Phasenwerte, heißt direkte Quantisierung $\psi_{\mathrm{DQ}}$. Die Quantisierung führt zu einer Verschlechterung der Rekonstruktion des Signals und zu geringerer Effizienz. Diese unerwünschten Effekte lassen sich durch einen Iterativen Fourier-Quantisierungs-Transformationsalgorithmus (IFQA) reduzieren. Der IFQA ist dem IFTA ähnlich, wobei eine schrittweise Einengung der Intervalle um die $Z$-Phasenlevel in der DPE-Ebene dazu führt, dass der Algorithmus am Ende eine $Z$-wertige Funktion $\psi_{\mathrm{IFQA}}$ liefert mit geringeren $r m s_{s}$-Werten und höherer Effizienz $\eta$ als $\psi_{\mathrm{DQ}}$. Der von der Iterationszahl $j$ und $Z$ abhängige Quantisierungsoperator $Q_{Z}^{j}$ ist in Referenz [54] beschrieben. Mit Erreichen der maximalen Iterationszahl $P$ projiziert $Q_{Z}^{P}$ auf die $Z$ Phasenlevel. Um das Beugungsbild des DPE in einer Signal-Ebene mit endlichem Abstand $z_{0}$ vom DPE zu rekonstruieren, muss eine sphärische Linse direkt hinter dem DPE plaziert werden. Der Abstand $z_{0}$ ist dann durch die Brennweite der Linse gegeben. Die Funktion der Linse kann durch Verwendung der Fresneltransformation (FRT) statt der Fouriertransformation (FT) 
als Propagationsoperator $\mathcal{P}$ in das DPE integriert werden. Unter Vernachlässigung des konstanten Terms in der Signal-Ebene folgt aus Gleichung (1.1):

$$
f(x, y)=F R T(g(u, v))=\exp \left[i \pi \lambda z_{0}\left(x^{2}+y^{2}\right)\right] F T\left(g(u, v) \exp \left[i \frac{\pi}{\lambda z_{0}}\left(u^{2}+v^{2}\right)\right]\right) .
$$

Durch Einführen abkürzender Schreibweisen für die quadratischen Phasenfaktoren

$$
\begin{aligned}
w\left(z_{0}, \mathbf{x}\right) & =\exp \left[i \pi \lambda z_{0}\left(x^{2}+y^{2}\right)\right] \\
W\left(z_{0}, \mathbf{u}\right)^{*} & =\exp \left[i \frac{\pi}{\lambda z_{0}}\left(u^{2}+v^{2}\right)\right]
\end{aligned}
$$

lassen sich die FRT und ihr Inverses kompakt schreiben:

$$
\begin{aligned}
F R T & =w\left(z_{0}, \mathbf{x}\right) F T W\left(z_{0}, \mathbf{u}\right)^{*} \\
F R T^{-1} & =W\left(z_{0}, \mathbf{u}\right) F T^{-1} w\left(z_{0}, \mathbf{x}\right)^{*} .
\end{aligned}
$$

Die Phasenfaktoren $W, w$ werden von den Operatoren $\mathcal{S}, \mathcal{G}$ nicht beeinflußt. In der SignalEbene bleibt auch die Phase $\phi^{j}=\bar{\phi}$ unverändert, so dass sich $w\left(z_{0}, \mathbf{x}\right)^{*} w\left(z_{0}, \mathbf{x}\right)=1$ in einem Transformationszyklus aufheben [6]. Das resultierende IFQA-Schema mit Einrechnen einer Fokuslänge $z_{0}$ durch Verwendung der FRT ist in Abbildung (1.5) gezeigt.

\section{Signal Ebene}

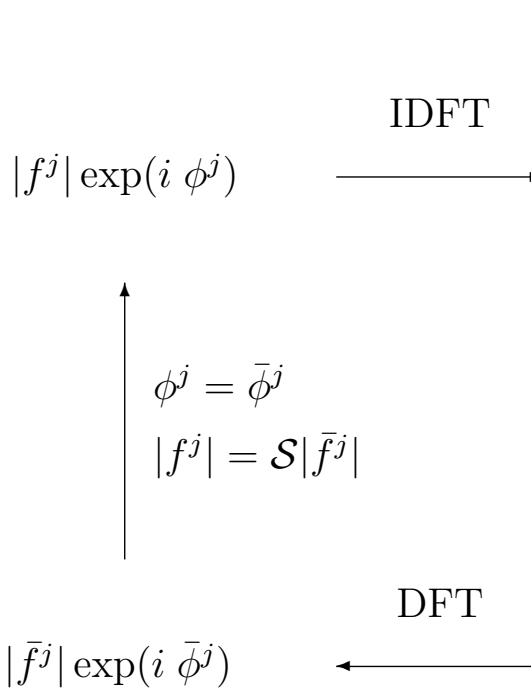

\section{DPE-Ebene}

\section{$|g|$ und $\psi$ aus IFTA}

$$
\left|g^{j-1}\right| \exp \left(i \psi^{j-1}\right)
$$

$$
\begin{aligned}
& \bar{\psi}^{j}=W^{*} Q_{Z}^{j} W \psi^{j-1} \\
& \left|\bar{g}^{j}\right|=\mathcal{G}\left(\left|g^{j-1}\right|\right)
\end{aligned}
$$

$$
\begin{gathered}
\left|\bar{g}^{j}\right| \exp \left(i \bar{\psi}^{j}\right) \\
\downarrow \mathrm{j}=\mathrm{P} \\
\text { quantisiertes } \psi_{\mathrm{IFQA}}
\end{gathered}
$$

Abbildung 1.5: IFQA-Schema 
Das Beugungsbild $f$ in der Signalebene kann um einen konstanten Vektor $\mathbf{x}_{\mathbf{v}}^{\prime}=\lambda z_{0} \mathbf{x}_{\mathbf{v}}$ verschoben werden, indem der Phasenfaktor $W\left(z_{0}, \mathbf{u}\right)^{*}$ durch $W\left(z_{0}, \mathbf{u}-\mathbf{x}_{\mathbf{v}}^{\prime}\right)^{*}$ ersetzt wird $[6]$.

\subsection{Der verwendete Excimer-Laser}

Excimer-Laser gehören zu der Gruppe der Gas-Laser. Der Name „Excimer“ ist ein Akronym für „excited dimer“", das an die angeregten zweiatomigen Moleküle erinnert, z.B. $\mathrm{Xe}_{2}$, die ursprünglich als Lasergas benutzt wurden. Moderne Excimer-Laser benutzen angeregte Edelgas-Halogen-Moleküle, z.B. KrF oder ArF, als aktives Medium aufgrund der höheren Effizienz. Die Anregung des aktiven Mediums wird durch gepulste Gasentladung zwischen zwei Elektroden bei einem Gasdruck von $1,5 \cdot 10^{5} \mathrm{~Pa}-6 \cdot 10^{6} \mathrm{~Pa}$ realisiert [4]. Diese Anregungstechnik führt zu hohen Pulsenergien und dem charateristischen Strahlquerschnitt der Excimer-Laser von ca. $1 \cdot 3 \mathrm{~cm}^{2}$. Im Rahmen dieser Arbeit wurden diffraktive Phasenelemente für $\mathrm{KrF}$ (Wellenlänge $\lambda=248 \mathrm{~nm}$ ) Excimer-Laser untersucht. Die Experimente zur optischen Rekonstruktion der DPE bei der Design-Wellenlänge sind mit einem Lambda Physik L1000 Excimer-Laser durchgeführt worden, dessen Resonator mit dem entsprechenden Gasgemisch gefüllt wurde.

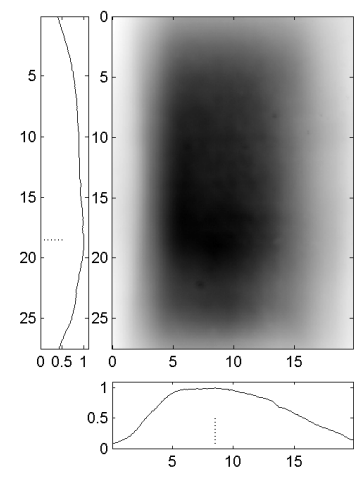

Nahfeld

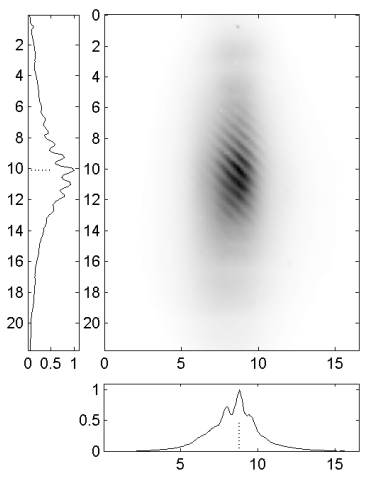

Fernfeld

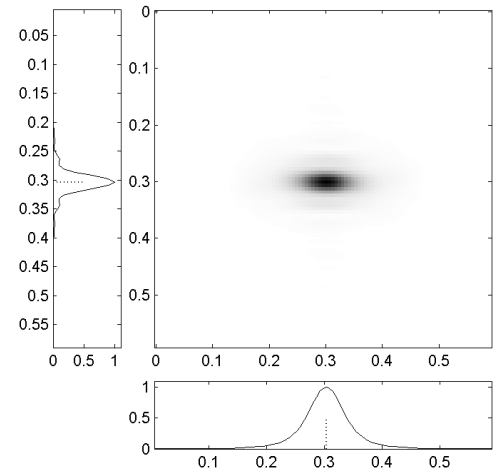

Kohärenzfunktion $|j|$ im Nahfeld

Abbildung 1.6: Nah- und Fernfeld des L1000 Excimer-Lasers.

Einfache Graustufenskala. Alle Achsen in mm.

Das Fernfeld ist in der Brennebene einer sphärischen Linse $(\mathrm{f}=1375 \mathrm{~mm})$ aufgenommen.

Charakteristisches Merkmal für die Strahlung der Excimer-Laser ist ihre vergleichsweise geringe Kohärenz und die daraus resultierende divergente Strahlpropagation (vgl. Kapitel 
5.6 in [40]). Die Strahleigenschaften in den beiden Achsen sind stark unterschiedlich. Die kurze Achse hat im Nahfeld einen annähernd gaussförmigen Querschnitt, die lange Achse ist stärker divergent (siehe Abbildung (1.6)).

Es ist möglich, die Resonatoren von Excimer-Lasern so zu justieren, dass man symmetrischere Nah- und Fernfeldprofile als in Abbildung (1.6) erhält. Die abgebildeten Profile sind aber durchaus „typisch“ für den L1000 und andere in der Materialbearbeitung eingesetzte Excimer-Laser. Das Fernfeld ist in der Brennebene einer sphärischen Linse $(\mathrm{f}=1375 \mathrm{~mm})$ aufgenommen worden. Mit dem verallgemeinerten Van-Cittert-ZernikeTheorem (vgl. Gleichung (2.18) und Referenz [22]) läßt sich aus der Fernfeldverteilung über eine inverse Fouriertransformation der Betrag der Kohärenzfunktion $j$ im Nahfeld berechnen. Aus Abbildung (1.6) kann für die kurze Laserachse eine Kohärenzlänge von ca. $200 \mu \mathrm{m}$ und für die lange ca. $100 \mu \mathrm{m}$ abgelesen werden. Die speziellen Strahleigenschaften werden in den nächsten Kapiteln im Design der DPE berücksichtigt.

\subsection{Homogenisierung durch Apertursegmentierung}

Die inhomogene Intensitätsverteilung der Excimer-Laser ist für viele Anwendungen unbrauchbar. Eine Möglichkeit, wenigstens in einer Ebene eine homogene Verteilung zu erhalten, ist die Verwendung refraktiver Zylinderlinsenhomogenisierer [41]. Sieben bis zehn Zylinderlinsenstäbe (Breite ca. 2-3mm) werden nebeneinander angeordnet. Dahinter werden identische Stäbe um 90 Grad gedreht positioniert. Der Strahl wird somit in quadratische Subaperturen segmentiert. Eine sphärische Linse hinter den gekreuzten Zylinderlinsen bewirkt, dass die Strahlung aus den verschiedenen Subaperturen ein gemeinsames Quadrat beleuchten. Die Überlagerung der zueinander inkohärenten Teilbündel aus verschiedenen Ausgangsregionen des Strahls mittelt Inhomogenitäten aus. Die schematische Zeichnung in Abbildung (1.7) verdeutlicht dies Prinzip.

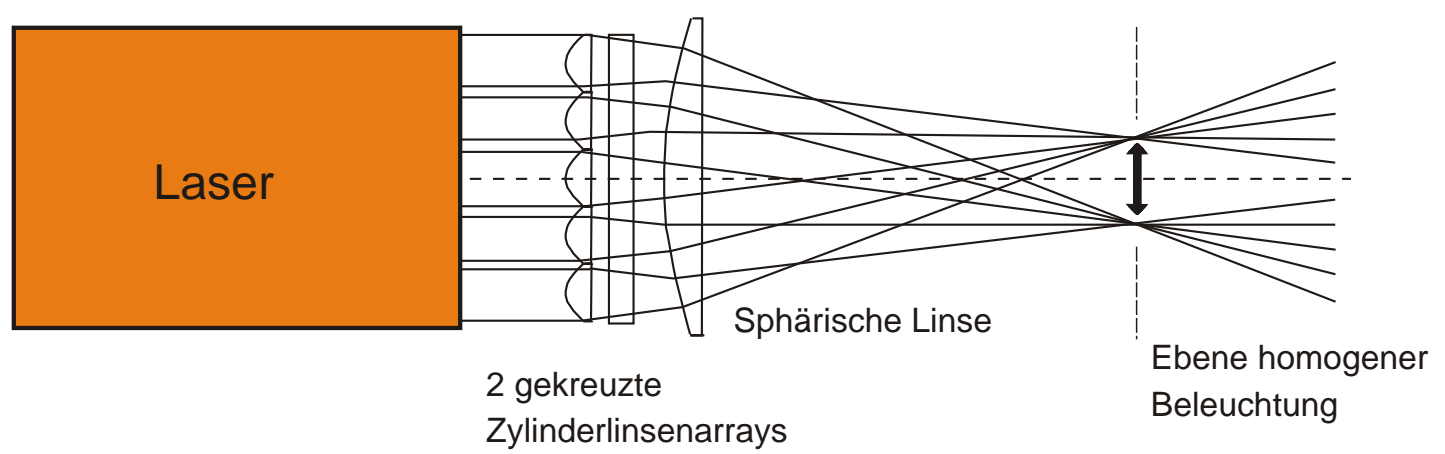

Abbildung 1.7: Prinzip der Homogenisierung durch Apertursegmentierung mit refraktiven Zylinderlisenarrays

Die laterale Ausdehnung der Subaperturen $\Delta u$ muss deutlich größer sein als die 
Kohärenzlänge der Laserstrahlung, sonst ruft das Gitter der Subaperturen unerwünschte Beugungseffekte hervor. Andererseits sollte $\Delta u$ nicht zu klein gewählt werden, um eine ausreichende Mittelung zu erreichen. Aus diesen Gründen liegt die Subaperturgröße meist im Bereich $2 \mathrm{~mm} \leq \Delta u \leq 3 \mathrm{~mm}$.

Die gekreuzten Zylinderlinsen lassen sich durch ein diffraktives Mikrolinsenarray ersetzen [43]. Mit diesem Prinzip - refraktiv oder diffraktiv realisiert - lassen sich jedoch nur rechteckige Strahlquerschnitte formen.

\subsection{Festlegung grundlegender Parameter der DPE und ihre Herstellung}

Die grundlegenden Parameter, die bei gegebener Übertragungslänge $z_{0}$ und Wellenlänge $\lambda$ vor der Berechnung eines DPE festgelegt werden müssen, sind:

1. Die Subaperturgröße $\Delta u$.

2. Die Pixelgröße $\delta u$ innerhalb einer Subapertur.

3. Die Anzahl der Phasenlevel $Z$.

Die Ausdehnung der Subaperturen beträgt $2 \mathrm{~mm} \leq \Delta u \leq 3 \mathrm{~mm}$ (vgl. Abschnitt (1.6)). Die Wahl der Pixelgröße $\delta u$ des DPE und der Anzahl $Z$ der Phasenlevel hängt von mehreren Faktoren ab:

Viele Anwendungen erfordern relativ große Beugungswinkel. Gemäß der skalaren Beugungstheorie wird die maximale Ausdehnung $\Delta x$ der signaltragenden 1. Beugungsordnung bestimmt durch die Pixelgröße $\delta u$, die Wellenlänge $\lambda$ und die Übertragungslänge $z_{0}[39]$ :

$$
\Delta x=\frac{\lambda z_{0}}{\delta u}
$$

Die untere Grenze für $\delta u$ wird definiert durch die Gültigkeitsgrenze der geometrischen Näherung für die Ausbreitung der Strahlung vom tiefsten Phasenlevel bis zum höchst gelegenen. Es darf keinen „crosstalk“ zwischen den DPE-Pixeln geben. Diese untere Grenze wird für Grenzflächen mit Brechungsindices $n^{I}=1,5$ und $n^{I I}=1$

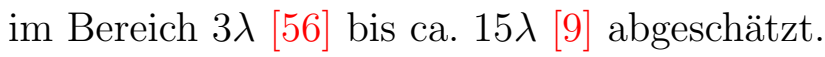

Der Rechenaufwand steigt trotz Verwendung des FFT-Algorithmus mit steigender Pixelzahl $N \cdot M$ stark an. Die Anzahl der benötigten "floating point" Rechenoperationen (flops) beträgt ca. $5 N M\left(\log _{2} N+\log _{2} M\right)$ für eine zweidimensionale FFTBerechnung [24]. 


\subsection{Festlegung grundlegender Parameter der DPE und ihre Herstellung}

Die Herstellung mehrstufiger $1 \cdot 3 \mathrm{~cm}^{2}$ großer DPE mit Auflösungen im $\mu \mathrm{m}$-Bereich stellt hohe Anforderungen an den Fabrikationsprozess.

Unter Berücksichtigung dieser Faktoren wurden $Z=4$ Phasenlevel bei einer Pixelgröße $\delta u=2,5 \mu \mathrm{m}$ gewählt. Fehler bei der Fabrikation wie Strukturgrößenfehler, MaskenPositionierfehler und Ätztiefenfehler wirken sich vor allem durch erniedrigte Beugungseffizienz im Signal aus [34]. Der Einfluß dieser Fehler nimmt mit größerer Stufenanzahl Z $\mathrm{zu}$.

Eine ausführliche Übersicht über die unterschiedlichen Verfahren der Herstellung diffraktiver Elemente in verschiedenen Materialien gibt Referenz [20]. Aufgrund der erforderlichen hohen Transmission bei kurzen Wellenlängen bieten sich für UV-Optiken benutzte Materialien, z.B. Quarzglas (Suprasil) oder $\mathrm{CaF}_{2}$, zur Verwendung für DPE für ExcimerLaser an. Quarzglas läßt sich mittels Elektronenstrahllithographie und Trockenätzverfahren präzise bearbeiten [32]. Die berechneten DPE sind mit dieser Technik am Institut für angewandte Physik der Uni Jena durch Mitarbeiter der Gruppe von Dr. Kley angefertigt worden. Eine schematische Darstellung der Prozessschritte zur Herstellung mehrstufiger DPE ist in Abbildung (1.8) gezeigt.

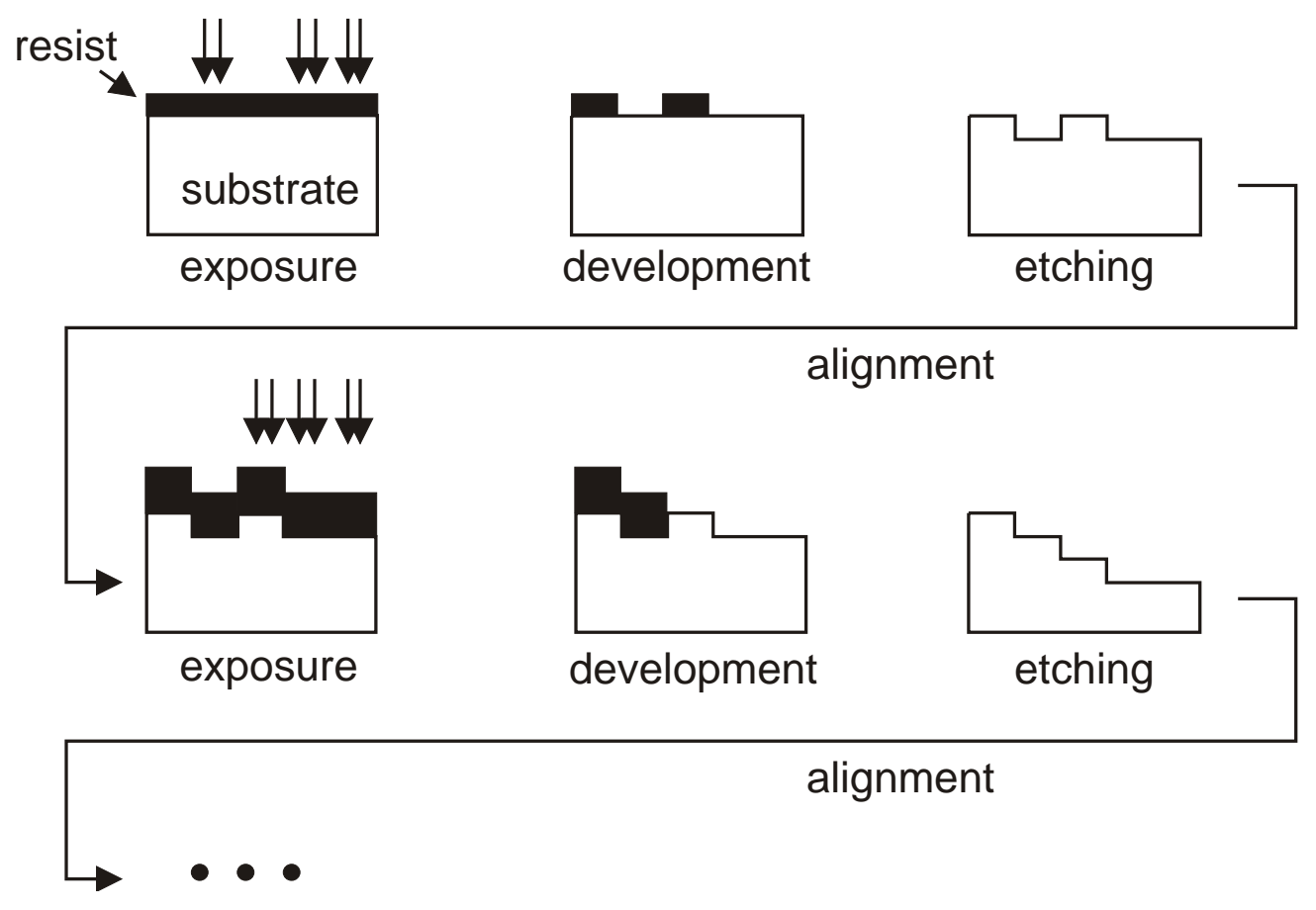

Abbildung 1.8: Herstellung eines mehrstufigen DPE durch wiederholten Lithographie-/Ätzprozess (nach [34])

In Abbildung (1.9) sind Raster-Elektronenmikroskopaufnahmen (REM) des mit dieser Technik in Quarzglas hergestellten DPE Nr. I gezeigt. 


\section{Grundlagen}
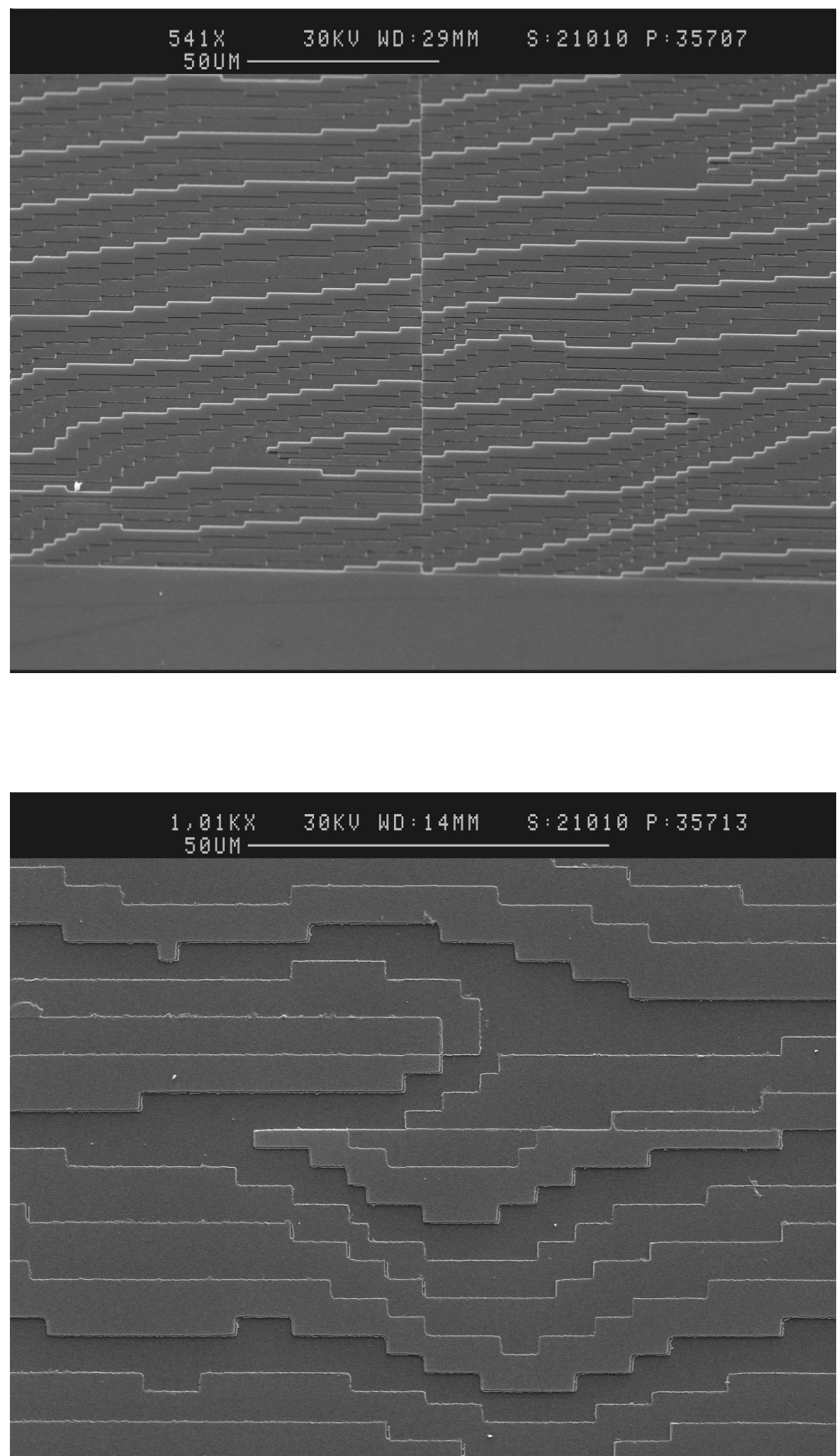

Abbildung 1.9: REM Bilder der Kontaktregion zweier Subaperturen und DPE-Rand (oben) und ein vergrößerter Ausschnitt (unten) des vierstufigen DPE Nr. I in Quarzglas mit einer Pixelgröße von $\delta u=2,5 \mu \mathrm{m}$. 


\section{Modellierung partiell kohärenter Strahlung}

Die Kohärenzgebiete der „Rohstrahlung“ eines Excimer-Lasers sind vergleichsweise klein (vgl. Abschnitt (1.5)), weshalb sich die Fraunhofer-Näherung nur bedingt zur Modellierung dieser Strahlung eignet. In diesem Kapitel wird die Theorie partiell-kohärenter Strahlung innerhalb der skalaren Beugungstheorie entwickelt, insbesondere die Theorie der quasi-homogenen, sekundären, planaren Quellen [8], [40]. Es wird gezeigt, dass die verwendete Excimer-Laserstrahlung die Kriterien dieser Quellen erfüllt.

\subsection{Das Strahlungsfeld als stochastischer Prozess}

Alle in diesem Kapitel benutzten komplexwertigen Funktionen $\left(f(\mathbf{x}), \mathbf{x} \in \mathbb{R}^{n}\right)$ seien hinreichend gutmütig oder sind durch solche zu approximieren, dass die benutzten mathematischen Sätze gelten. Es wird angenommen, das skalare Wellenfeld $E(\mathbf{x}, t)$ lasse sich durch einen ergodischen, stochastischen Prozeß beschreiben [44]. Die mutual coherence function $\Gamma$ ist dann definiert durch:

$$
\Gamma\left(\mathbf{x}_{\mathbf{1}}, \mathbf{x}_{\mathbf{2}}, \tau\right):=\left\langle E\left(\mathbf{x}_{\mathbf{1}}, t+\tau\right) E^{*}\left(\mathbf{x}_{\mathbf{2}}, t\right)\right\rangle=\lim _{T \rightarrow \infty} \frac{1}{2 T} \int_{-T}^{T} E\left(\mathbf{x}_{\mathbf{1}}, t+\tau\right) E^{*}\left(\mathbf{x}_{\mathbf{2}}, t\right) \mathrm{d} t .
$$

Die normierte Version von $\Gamma$ heißt degree of coherence $\gamma$ :

$$
\gamma\left(\mathbf{x}_{1}, \mathbf{x}_{2}, \tau\right):=\frac{\Gamma\left(\mathbf{x}_{1}, \mathbf{x}_{\mathbf{2}}, \tau\right)}{\Gamma\left(\mathbf{x}_{1}, \mathbf{x}_{1}, 0\right) \Gamma\left(\mathbf{x}_{2}, \mathbf{x}_{2}, 0\right)} \quad \Rightarrow \quad 0 \leq \gamma \leq 1
$$

Diese Größen beschreiben die räumlichen und zeitlichen Kohärenzeigenschaften des Wellenfeldes. Für die rein räumlichen Anteile, d.h. $\tau=0$, wird eine eigene Funktion eingeführt, die mutual intensity $J$ und deren normierte Version $j$, der equal-time degree of coherence:

$$
\begin{aligned}
J\left(\mathbf{x}_{1}, \mathbf{x}_{2}\right) & =\Gamma\left(\mathbf{x}_{1}, \mathbf{x}_{2}, 0\right) \\
j\left(\mathbf{x}_{1}, \mathbf{x}_{\mathbf{2}}\right) & =\frac{J\left(\mathbf{x}_{1}, \mathbf{x}_{\mathbf{2}}\right)}{J\left(\mathbf{x}_{1}, \mathbf{x}_{1}\right) J\left(\mathbf{x}_{2}, \mathbf{x}_{2}\right)} .
\end{aligned}
$$

Für diese Größen werden abkürzende Schreibweisen eingeführt:

$$
\begin{aligned}
\Gamma_{12}(\tau) & =\Gamma\left(\mathbf{x}_{\mathbf{1}}, \mathbf{x}_{\mathbf{2}}, \tau\right) \\
\gamma_{12}(\tau) & =\gamma\left(\mathbf{x}_{\mathbf{1}}, \mathbf{x}_{\mathbf{2}}, \tau\right) \\
J_{12} & =J\left(\mathbf{x}_{\mathbf{1}}, \mathbf{x}_{\mathbf{2}}\right) \\
j_{12} & =j\left(\mathbf{x}_{\mathbf{1}}, \mathbf{x}_{\mathbf{2}}\right) .
\end{aligned}
$$


Die Funktion $\Gamma$ kann durch $J$ ausgedrückt werden, falls die quasi-monochromatische Näherung erfüllt ist, d.h.:

$$
\frac{\Delta \lambda}{\lambda_{0}} \ll 1, \quad \text { mit } \lambda_{0}=\text { mittlere Wellenlänge, } \Delta \lambda=\text { Wellenlängenintervall, }
$$

und die betrachteten Zeitdifferenzen $|\tau|$ klein gegen den Kehrwert des Frequenzintervalls $\Delta \nu=\left(\nu_{0}-\nu\right)$ sind $|\tau| \ll 1 / \Delta \nu$, ausgedrückt in Wegunterschieden $\Delta S$ bedeutet das:

$$
|\Delta S|=\left|s_{2}-s_{1}\right|=c|\tau| \ll c \frac{1}{\Delta \nu}=\frac{\lambda_{0}^{2}}{\Delta \lambda} .
$$

In diesem Fall gilt ([8], Section 10.4):

$$
\Gamma_{12}(\tau) \approx J_{12} e^{2 \pi i \nu_{0} \tau}
$$

Goodman ([22], Section 7.2.2) nähert $\Gamma_{12}$ unter der Voraussetzung (2.6) durch:

$$
\Gamma_{12}(\tau) \approx J_{12}
$$

Die Bedingungen (2.5) und (2.6) sind für die verwendete Strahlung (Abschnitt (1.5)) und den verwendeten Aufbau erfüllt. Die Bandbreite der Excimer-Laserstrahlung beträgt ca. $1 \mathrm{~nm}[4]$, und damit gilt:

$$
\frac{\Delta \lambda}{\lambda_{0}}=\frac{1 \mathrm{~nm}}{248 \mathrm{~nm}} \ll 1
$$

Der maximale Wegunterschied ist durch die Subaperturgröße $\Delta u$ und die Übertragungslänge $z_{0}$ (vgl. Abschnitt 3.2) gegeben:

$$
|\Delta S| \approx \frac{(\Delta u)^{2}}{z_{0}}=\frac{(2,5 \mathrm{~mm})^{2}}{1250 \mathrm{~mm}}=5 \mu \mathrm{m} \ll 60 \mu \mathrm{m}=\frac{\lambda_{0}^{2}}{\Delta \lambda} .
$$

\subsection{Die Kohärenzfunktion $j$}

Die Kohärenzfunktion $j\left(\mathbf{x}_{\mathbf{1}}, \mathbf{x}_{\mathbf{2}}\right)$ quasi-monochromatischer Strahlung kann durch Youngs Interferenz-Experiment (siehe Abbildung (2.1)) vollständig bestimmt werden [40]. Der Betrag von $j_{12}$ ist gegeben durch die Sichtbarkeit $V$ (visibility) des Interferenzmusters im Punkt $P(\mathbf{r})$.

$$
\begin{aligned}
V(P) & =\frac{I_{\max }-I_{\min }}{I_{\max }+I_{\min }} \\
\left|j\left(\mathbf{x}_{\mathbf{1}}, \mathbf{x}_{\mathbf{2}}\right)\right| & =\frac{I\left(P_{1}\right)+I\left(P_{2}\right)}{2 \sqrt{I\left(P_{1}\right)} \sqrt{I\left(P_{2}\right)}} V(P)
\end{aligned}
$$




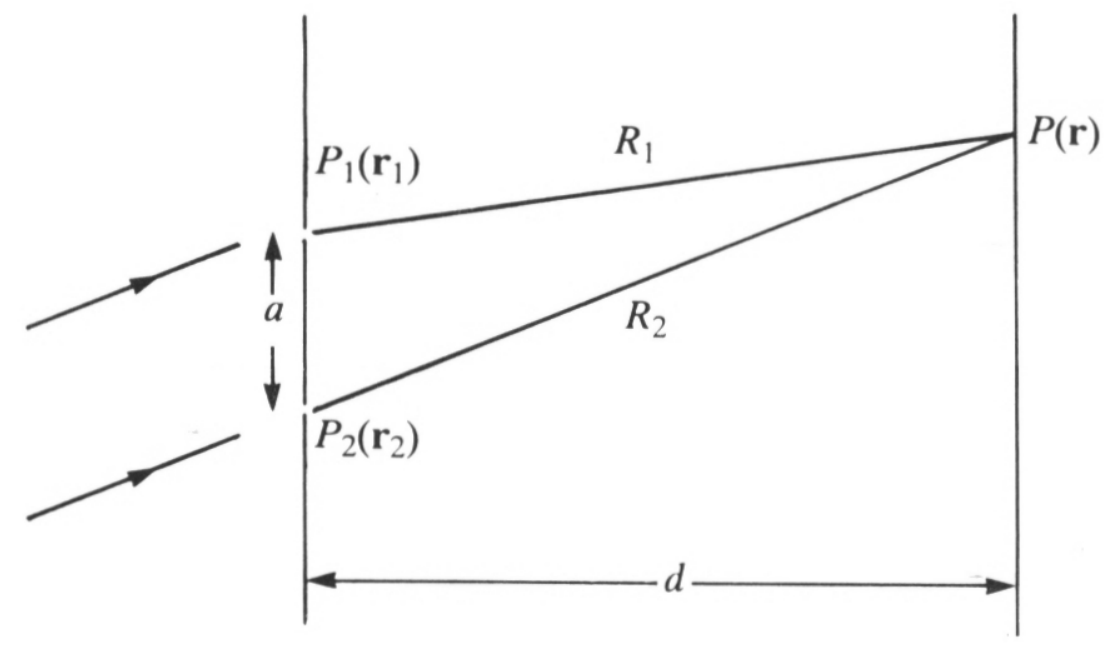

Abbildung 2.1: Zur Notation bei Youngs Interferenz-Versuch (aus [40]).

Die Phase von $j_{12}$ bestimmt die Verschiebung $x$ des Interferenzbildes in $P$ relativ zu dem Interferenzbild bei Verwendung monochromatischer, gleichphasiger Strahlung. In der paraxialen Näherung $\left(R_{1} \simeq R_{2}\right)$ gilt:

$$
x=\frac{a}{d} \frac{\lambda_{0}}{2 \pi} \arg j\left(\mathbf{x}_{\mathbf{1}}, \mathbf{x}_{2}\right)
$$

Kommt der Abstand $a$ der beiden Pinholes in die Größenordnung der Ausdehnung der Subaperturen $\Delta u$ ist der Betrag von $j_{12}$ schon so klein, dass kein Interferenzmuster mehr zu beobachten ist. Dies rechtfertigt die Modellierung der Strahlung aus verschiedenen Subaperturen als inkohärent zueinander. Die Phase von $j_{12}$ wird vernachlässigt, aber die Verkippung der Hauptstrahlrichtungen dieser inkohärenten Teilstrahlen erweist sich als wichtiger Effekt.

\subsection{Intensität im Fernfeld und Betrag der Kohärenz- funktion $j$}

Das Konzept der partiellen Kohärenz quasi-homogener, planarer, sekundärer Quellen soll nun zur Beschreibung des Beugungsbildes eines dünnen optischen Elements, dessen Transmission durch die komplexwertige Funktion $g(u, v)$ gegeben ist, angewendet werden. Die Intensitätsverteilung $I\left(x^{\prime}, y^{\prime}\right)$, die durch Beleuchtung des Elements mit partiell-kohärenter Strahlung in einer Ebene hinter dem Element entsteht, ist durch das Hopkins-Integral [28] gegeben. Falls die Bedingungen (2.5,2.6) erfüllt sind, kann das Hopkins-Integral verein- 
facht werden $\mathrm{zu}[22]$ :

$$
\begin{aligned}
I\left(x^{\prime}, y^{\prime}\right)=\iiint_{-\infty}^{\infty} & K\left(x^{\prime}, y^{\prime} ; u_{1}, v_{1}\right) K^{*}\left(x^{\prime}, y^{\prime} ; u_{2}, v_{2}\right) g\left(u_{1}, v_{1}\right) g^{*}\left(u_{2}, v_{2}\right) J\left(u_{1}, v_{1} ; u_{2}, v_{2}\right) \\
& \mathrm{d} u_{1} \mathrm{~d} v_{1} \mathrm{~d} u_{2} \mathrm{~d} v_{2},
\end{aligned}
$$

wobei $J\left(u_{1}, v_{1} ; u_{2}, v_{2}\right)$ die Kohärenzeigenschaften der Strahlung in der Ebene des Elements beschreibt und $K$ die amplitude spread function des optischen Systems ist. Bei der Propagation durch den freien Raum kann für $K$ in der Paraxialen Näherung für das Fernfeld der Fresnel- oder der Fraunhofer-Integrationskern eingesetzt werden. Durch Einfügen einer langbrennweitigen Linse hinter das dünne optische Element wird die Gültigkeit der Fraunhofer-Näherung in der Brennebene der Linse sichergestellt. Für die weiteren Betrachtungen wird deshalb der Fraunhoferkern benutzt:

$$
K\left(x^{\prime}, y^{\prime} ; u, v\right)=\frac{\exp \left(i \frac{2 \pi}{\lambda_{0}} z\right)}{i \lambda_{0} z} \exp \left(i \frac{\pi}{\lambda_{0} z}\left(x^{\prime 2}+y^{\prime 2}\right)\right) \exp \left(-2 \pi i\left(\frac{x^{\prime}}{\lambda_{0} z} u+\frac{y^{\prime}}{\lambda_{0} z} v\right)\right),
$$

wobei $z$ der Abstand der beiden Ebenen ist. Die Gleichung (2.9) wird damit zu:

$$
\begin{aligned}
I\left(x^{\prime}, y^{\prime}\right) & =\frac{1}{\lambda_{0}^{2} z^{2}} \iiint_{-\infty}^{\infty} \int g\left(u_{1}, v_{1}\right) g^{*}\left(u_{2}, v_{2}\right) J\left(u_{1}, v_{1} ; u_{2}, v_{2}\right) \\
& \exp \left(-2 \pi i\left(\frac{x^{\prime}}{\lambda_{0} z} u_{1}+\frac{y^{\prime}}{\lambda_{0} z} v_{1}\right)\right) \exp \left(2 \pi i\left(\frac{x^{\prime}}{\lambda_{0} z} u_{2}+\frac{y^{\prime}}{\lambda_{0} z} v_{2}\right)\right) \mathrm{d} u_{1} \mathrm{~d} v_{1} \mathrm{~d} u_{2} \mathrm{~d} v_{2}
\end{aligned}
$$

Dieses Vierfach-Integral kann für quasi-homogene, sekundäre, planare Quellen (siehe [13], [22]) in zwei Zweifach-Integrale zerlegt werden. Für eine quasi-homogene Quelle variiert der Kohärenzgrad $j$ erheblich schneller als die im wesentlichen konstante Intensität. Es werden neue Schwerpunkt- und Differenzvariablen für $u$ und $v$ eingeführt:

$$
\begin{array}{ll}
\Delta u=u_{1}-u_{2}, & \bar{u}=\frac{1}{2}\left(u_{1}+u_{2}\right) \\
\Delta v=v_{1}-v_{2}, & \bar{v}=\frac{1}{2}\left(v_{1}+v_{2}\right) .
\end{array}
$$

Eine quasi-homogene, sekundäre, planare Quelle ist definiert als Strahlungsfeld, dessen Mutual Intensity $J$ sich in der $u, v$-Ebene schreiben läßt als:

$$
J\left(u_{1}, v_{1} ; u_{2}, v_{2}\right)=I_{0} j(\Delta u, \Delta v) .
$$

Mit (2.12), (2.13) wird Gleichung (2.11) zu:

$$
\begin{aligned}
I\left(x^{\prime}, y^{\prime}\right)= & \frac{I_{0}}{\lambda_{0}^{2} z^{2}} \iiint_{-\infty}^{\infty} \int g\left(\bar{u}+\frac{\Delta u}{2}, \bar{v}+\frac{\Delta v}{2}\right) g^{*}\left(\bar{u}-\frac{\Delta u}{2}, \bar{v}-\frac{\Delta v}{2}\right) \\
& j(\Delta u, \Delta v) \exp \left(-2 \pi i\left(\frac{x^{\prime}}{\lambda_{0} z} \Delta u+\frac{y^{\prime}}{\lambda_{0} z} \Delta v\right)\right) \mathrm{d} \Delta u \mathrm{~d} \Delta v \mathrm{~d} \bar{u} \mathrm{~d} \bar{v} .
\end{aligned}
$$


Die beiden Integrationen über die Schwerpunktkoordinaten $\bar{u}, \bar{v}$ können nun ausgeführt werden. Sie liefern gerade die Autokorrelation der Pupillenfunktion $g$ :

$$
(g \otimes g)(\Delta u, \Delta v)=\int_{-\infty}^{\infty} \int_{-} g\left(\bar{u}+\frac{\Delta u}{2}, \bar{v}+\frac{\Delta v}{2}\right) g^{*}\left(\bar{u}-\frac{\Delta u}{2}, \bar{v}-\frac{\Delta v}{2}\right) \mathrm{d} \bar{u} \mathrm{~d} \bar{v} .
$$

Somit vereinfacht sich (2.14) zu:

$$
\begin{aligned}
I\left(x^{\prime}, y^{\prime}\right)= & \frac{I_{0}}{\lambda_{0}^{2} z^{2}} \iint_{-\infty}^{\infty}(g \otimes g)(\Delta u, \Delta v) j(\Delta u, \Delta v) \\
& \quad \exp \left(-2 \pi i\left(\frac{x^{\prime}}{\lambda_{0} z} \Delta u+\frac{y^{\prime}}{\lambda_{0} z} \Delta v\right)\right) \mathrm{d} \Delta u \mathrm{~d} \Delta v .
\end{aligned}
$$

Dieses Integral ist nun nur noch die zweidimensionale Fouriertransformation des Produktes aus $(g \otimes g)$ und $j$. Einführen von Raumfrequenzen $\left(x=x^{\prime} /\left(\lambda_{0} z\right)\right.$, usw.) und Benutzen der Fouriertheoreme führt auf:

$$
\begin{aligned}
I(x, y) \propto & F T[(g \otimes g) j](x, y) \\
& \swarrow \text { Faltungstheorem } \\
& =F T[(g \otimes g)](\tilde{x}, \tilde{y}) * F T[j](\tilde{x}, \tilde{y}) \\
& \swarrow \text { Korrelationstheorem } \\
& =|F T[g](\tilde{x}, \tilde{y})|^{2} * F T[j](\tilde{x}, \tilde{y}) \\
& =I_{\operatorname{coh}}(\tilde{x}, \tilde{y}) * F T[j](\tilde{x}, \tilde{y})
\end{aligned}
$$

Für den Grenzfall komplett kohärenter Beleuchtung $j=1$ wird die Fouriertranformierte von $j$ zur Deltadistribution $\delta(\tilde{x}, \tilde{y})$. Die Schreibweise $I_{\text {coh }}$ in der letzten Zeile verdeutlicht, dass das Ergebnis dann mit der Fraunhofer-Näherung der kohärenten Beugungstheorie zusammenfällt.

Die Kohärenzfunktion $j(\Delta u, \Delta v)$ in der $(u, v)$-Ebene kann mit Hilfe des verallgemeinerten Van Cittert-Zernike Theorems durch die Fernfeld-Intensitätsverteilung $I_{\mathrm{f}}(x, y)$ des beleuchtenden Wellenfeldes ausgedrückt werden. Carter und Wolf ([13], Gleichung (4.6)) leiten den folgenden Ausdruck für den spectral degree of coherence $\mu$ ab. Es kann jedoch gezeigt werden [18], dass unter den Voraussetzungen $(2.5,2.6)$ die Näherung gilt $j=\mu$. Es gilt also:

$$
I_{\mathrm{f}}(x, y)=C \times F T[j(\Delta u, \Delta v)](x, y) .
$$

In die Konstante $C$ gehen die Wellenlänge $\lambda_{0}$, der Abstand $z$ und die Gesamtintensität in der $(u, v)$-Ebene ein. Verwendung der Gleichungen $(2.17,2.18)$ und Ersetzen der Proportionalitätskonstanten durch geeignete Normierung führt schließlich auf:

$$
I(x, y)=I_{\operatorname{coh}}(\tilde{x}, \tilde{y}) * I_{\mathrm{f}}(\tilde{x}, \tilde{y}) .
$$




\subsection{Hauptstrahlrichtungen im Nahfeld}

Die zueinander inkohärenten Strahlenbündel des Excimer-Lasers sind divergent. Diese unterschiedlichen Hauptstrahlrichtungen lassen sich durch Shack-Hartmann-Messungen bestimmen [45], [46]. In Abbildung (2.2) ist das Messprinzip eines Shack-Hartmann-Sensors dargestellt. Die Strahlung des Lasers trifft auf ein rechteckiges Pinhole-Gitter, dessen

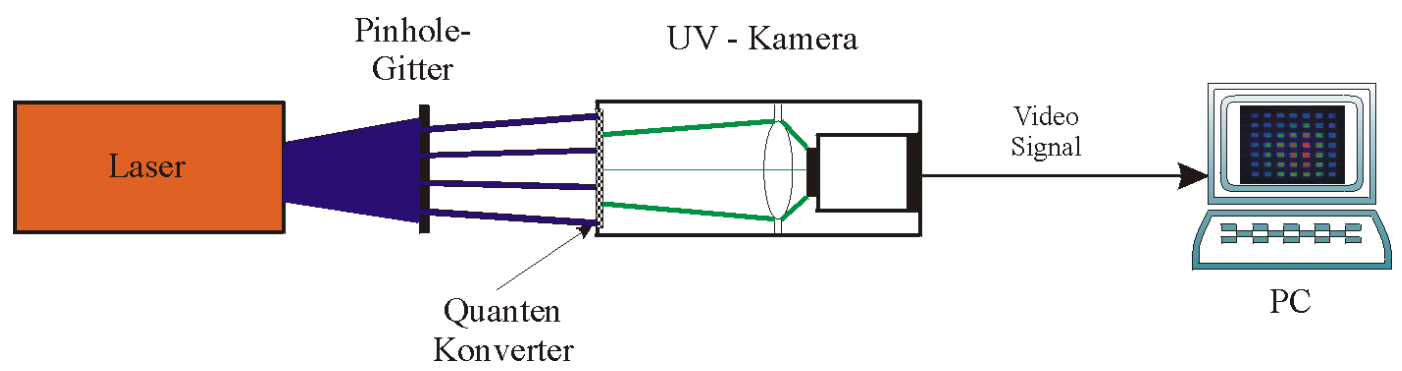

Abbildung 2.2: Prinzip des Shack-Hartmann Sensors

Löcher in einem Abstand von $1 \mathrm{~mm}$ zueinander positioniert sind. Die durch die Löcher propagierende Strahlung trifft auf einen Quantenkonverter, dessen emittiertes Licht auf einen CCD-Chip abgebildet wird. Der Sensor wird kalibriert, indem das Spot-Muster eines stark aufgeweiteten HeNe-Lasers aufgenommen wird, dessen Wellenfront als eben angenommen wird. Mit dem Excimer-Laser wird ein über ein oder mehrere Pulse gemitteltes Spot-Muster aufgenommen. Die Verschiebung der Schwerpunkte der Spots des Excimer-Lasers relativ zu denen des HeNe-Lasers ermöglicht die Berechnung einer zeitlich gemittelten „Wellenfrontcharakteristik“ [46]. Bei der Messung ist darauf zu achten, dass kein Licht aus einem Pinhole auf die zu einem benachbarten Pinhole gehörende Detektorfläche fällt und damit die Schwerpunktsberechnung der Spots verfälscht. Auf dem rechteckigen Messgitter wird die Wellenfrontcharakteristik nach Legendrepolynomen entwickelt, so dass sie auch auf beliebigen Zwischenpunkten ausgerechnet werden kann. Die Hauptstrahlrichtungen stehen senkrecht auf der Wellenfrontcharakteristik. Eine ShackHartmann Messung im Nahfeld des L1000 Excimer-Lasers (vgl. Abschnitt (1.5)) ist in Abbildung (2.3) gezeigt. 

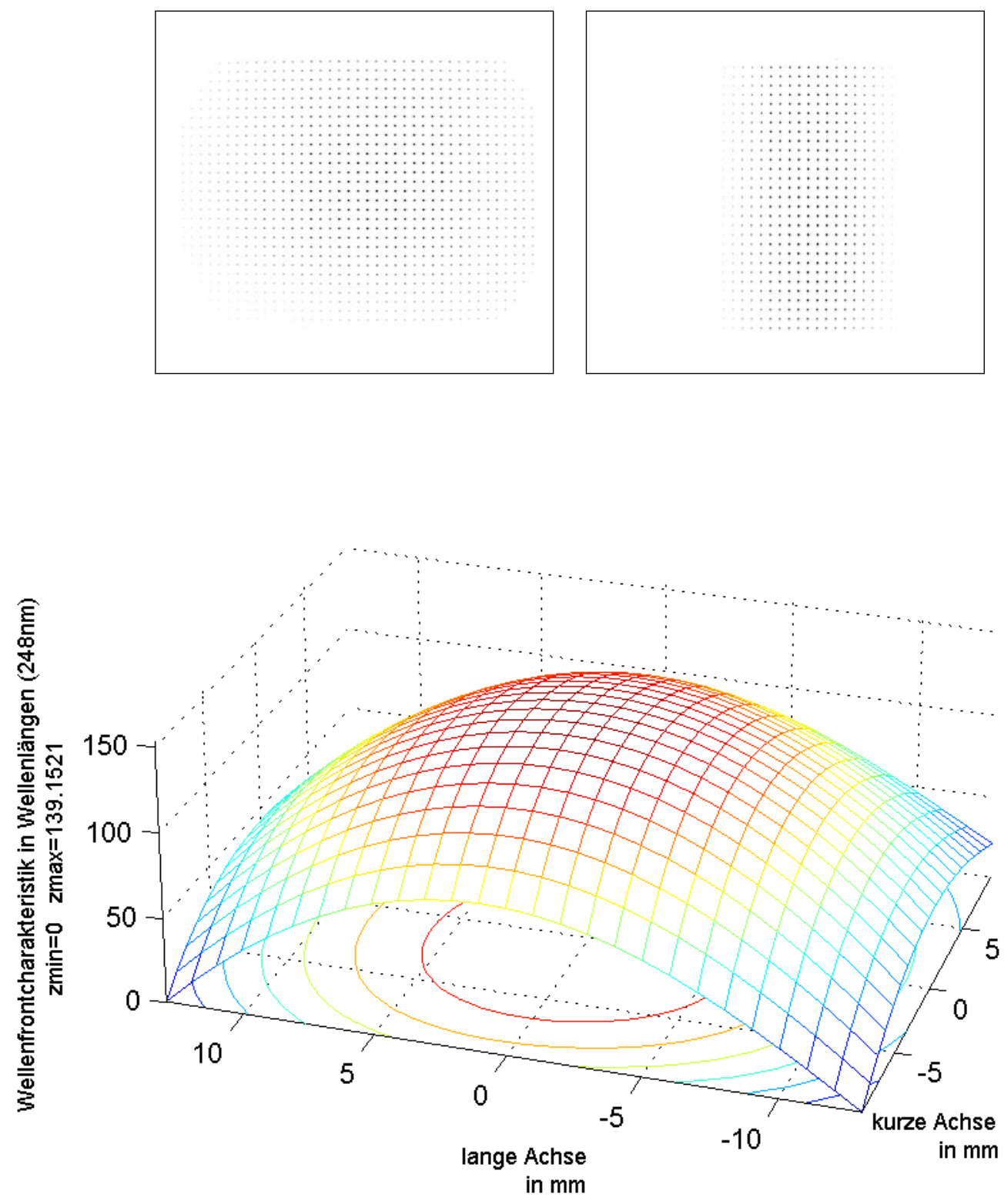

Abbildung 2.3: Shack-Hartmann Messung:

Links oben: Spot-Muster des aufgeweiteten HeNe-Lasers zur Kalibration. Rechts oben: Spot-Muster im Nahfeld des L1000.

Unten: Aus obigen Messungen berechnete Wellenfrontcharakteristik des L1000. 


\section{Strahlformung und Homogenisierung mit DPE}

In diesem Kapitel wird das in Abschnitt (1.4) beschriebene IFTA/IFQA-Designkonzept auf die Formung partiell-kohärenter Laserstrahlung übertragen. Am Beispiel der Strahlformung des rechteckigen Strahlquerschnitts eines Excimer-Lasers in einen runden Strahlquerschnitt durch ein DPE werden der Einfluss der Divergenz und der Kohärenz der Laserstrahlung auf die optische Rekonstruktion des DPE untersucht. Dies DPE wird als DPE Nr. I bezeichnet. In den folgenden Kapiteln werden weitere Konzepte zur Verbesserung der Strahlformung entwickelt und in Form der DPE Nr. II-IV realisiert.

\subsection{Prinzip des optischen Systems}

Zylinderlinsen-Homogenisierer ermöglichen die Formung homogener, rechteckiger Strahlquerschnitte (vgl. Abschnitt (1.6)). Der Einsatz Diffraktiver Phasenelemente, bestehend aus verschiedenen Subaperturen, auch „Multifacettenelemente“ genannt, dient zur Homogenisierung des Excimer-Laserstrahls. Die Strahlformung innerhalb jeder Subapertur in komplexe Geometrien kann viele neue Anwendungen ermöglichen.

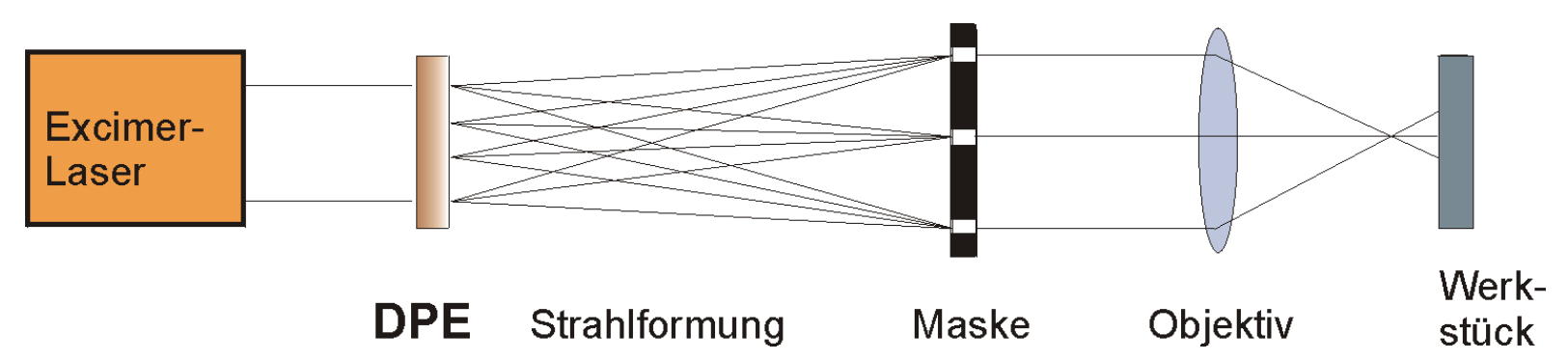

Abbildung 3.1: Prinzipieller Einsatz eines DPE für die Mikromaterialbearbeitung

In Abbildung (3.1) ist ein schematischer Aufbau für den Einsatz eines DPE in der Mikromaterialbearbeitung gezeigt. Durch wellenoptische Strahlformung werden aus dem einfallenden Strahl in jeder Subapertur drei Spots in die Maskenebene propagiert. Der laterale Versatz der einzelnen Subaperturen muss für die passgenaue Überlagerung des gewünschten Signals in der Maskenebene berücksichtigt werden. Um scharfe Kanten bei 
der Abbildung zu erreichen, wird die transmissive Struktur der Maske durch das DPE mit geringem Überlapp ausgeleuchtet. Ein Objektiv bildet die Maskenstruktur telezentrisch auf das Werkstück ab.

\subsection{Design und Berechnung des DPE Nr. I}

Ziel war es, ein DPE zu berechnen und herzustellen, das aus dem rechteckigen, inhomogenen Strahl des L1000 einen kreisförmigen Strahlquerschnitt (5 mm Durchmesser) mit möglichst homogenem, steilkantigem Top-Hat-Profil formt.

Das DPE Nr. I ist aus 11 - 11 Subaperturen zusammengesetzt. Durch jede Subapertur wird ein 5-mm Spot geformt. Alle Spots überlagern sich auf der optischen Achse des Gesamtsystems. Die Berechnung jeder Subapertur erfolgte mit dem in Abschnitt (1.4) beschriebenen zweistufigen IFTA/IFQA-Algorithmus mit folgenden Parametern:

\begin{tabular}{lc|lrl} 
IFTA-Parameter DPE Nr. I & & IFQA-Parameter DPE Nr. I & & \\
\hline Pixelzahl N (vert.) & 996 & Pixelgröße DPE $\delta u$ & 2,5 & $\mu \mathrm{m}$ \\
Pixelzahl M (hor.) & 996 & Subaperturgröße $\Delta u$ & 2,49 & $\mathrm{~mm}$ \\
max. Iterationszahl & 110 & Übertragungslänge $z_{0}$ & 1,25 & $\mathrm{~m}$ \\
Abbruchkriterium $\mathrm{rms}_{s}$ & $10^{-5}$ & Pixelgröße Signal $\delta x$ & 124,5 & $\mu \mathrm{m}$ \\
& & Iterationszahl P & 45 & \\
& & Stufenzahl $Z$ & 4 &
\end{tabular}

Um die Überlagerung der einzelnen Spots zu erreichen, müssen die Signale der äußeren Subaperturen zur optischen Achse des Gesamt-DPE abgelenkt werden. Dies wird erreicht durch das zusätzliche Einrechnen einer linearen Phase in jede Subapertur $(i, j)$ :

Sei $\mathbf{K}_{i j}=\left(\begin{array}{l}i \\ j\end{array}\right)$ der Koordinatenvektor der Subapertur in der i-ten Zeile und der j-ten Spalte, und $\hat{\mathbf{K}}_{i j}$ der entsprechende Einheitsvektor. Die Subapertur auf der optischen Achse habe den Koordinatenvektor $\mathbf{K}_{00}=\left(\begin{array}{l}0 \\ 0\end{array}\right)$. Das Beugungsbild $f$ in der Signalebene muss dann um den geometrischen Versatz jeder Subapertur $\left\langle\mathbf{K}_{i j}, \Delta \mathbf{u}\right\rangle$ zurück verschoben werden:

$$
\mathbf{x}_{\mathbf{v}_{i j}}=\left\langle\mathbf{K}_{i j}, \Delta \mathbf{u}\right\rangle \hat{\mathbf{K}}_{i j} \in \mathbb{R}^{2}
$$

In den quadratischen Phasenfaktor $W\left(z_{0}, \mathbf{u}-\mathbf{x}_{\mathbf{v}}^{\prime}\right)^{*}$ der IFQA-Rechnung (vgl. Abschnitt (1.4.2)) muss der Vektor $\mathbf{x}^{\prime} \mathbf{v}_{i j}$ aus Gleichung (3.1) eingesetzt werden.

Da es keinen bandbegrenzten Phasenfaktor gibt, der die gestellte Strahlformungsaufgabe erfüllt (vgl. Abschnitte (1.2), (1.3) und Referenz [53]), somit der iterative Algorithmus nur Näherungen an eine Lösung liefert, müssen Abweichungen vom Soll-Signal innerhalb und außerhalb der Indexmenge $\mathbb{I}$ dessen Trägers akzeptiert werden. 


\begin{tabular}{c|c|c|l|} 
& \multicolumn{3}{|c|}{ DPE Nr. I } \\
& IFTA & IFQA & \\
\hline$r m s_{s}$ & $10^{-5}$ & $3 \cdot 10^{-3}$ & \\
$\eta$ & $92 \%$ & $75 \%$ & $\eta(\mathrm{Z})=61 \%$
\end{tabular}

Tabelle 3.1: Über 121 Subaperturen gemittelte IFTA/IFQA-Werte für Objektfehler $r m s_{s}$ und Effizienz $\eta$.

In Tabelle (3.1) sind die aus den IFTA/IFQA-Rechnungen resultierenden Werte für den Objektfehler $r m s_{s}$ (A.3) und für die Effizienz $\eta$ (A.4) zusammengestellt. Der Wert für die Effizienz $\eta$ aus der IFQA-Rechnung berücksichtigt nicht die Strahlung, die in höhere Beugungsordnungen propagiert. Das DPE wird durch $N \cdot M$ Pixel modelliert, ebenso die 1. Beugungsordnung in der Signal-Ebene. Die auf dem Parseval-Theorem beruhende Normierung (1.11) bezieht sich auf diese beiden Matrizen. Aus Gleichung (1.10) läßt sich ein Faktor errechnen, der den relativen Intensitätsanteil in der 1. Beugungsordnung in Abhängigkeit der Stufenanzahl $Z$ angibt. Um den Anteil der Gesamtintensität abzuschätzen, der abzüglich der in höhere Beugungsordnungen propagierenden Strahlung, in der gewünschten Geometrie der 1. Beugungsordnung verbleibt, wird der $\eta$ (IFQA)-Wert mit diesem Faktor multipliziert. Für $Z=4$ Stufen und die 1. Beugungsordnung $(m=1)$ läßt sich aus der Tabelle (1.1) der Faktor 0,81 ablesen. Die resultierende Abschätzung $\eta(\mathrm{Z})$ ergibt sich dann als: $\eta(Z)=0,81 \cdot \eta($ IFQA $)$.

Dem Durchmesser $(5 \mathrm{~mm})$ des vorgegebenen Spots $\left|f^{0}\right|$ entsprechen 40 Pixelausdehnungen $\delta x$ in der Signalebene. In Abbildung (3.2) sind der vorgegebene Spot und die IFQA-Lösung gezeigt. Im mittleren Bild der IFQA-Lösung einer Subapertur ist in den Querschnitten der Wert für $\eta($ IFQA $)=75 \%$ direkt ablesbar. Außerhalb des Trägers des Spots ist ein grauer Schleier von Schmutzlicht erkennbar, der sich auch über die Grenzen des dargestellten 60 - 60 Pixel großen Ausschnitts erstreckt. In diesem Schmutzlicht stecken die fehlenden $25 \%$ der Intensität. Im Querschnitt sind minimale Abweichungen vom Top-Hat-Profil zu erkennen.

Im rechten Bild der inkohärenten Überlagerung der 121 IFQA-Lösungen mitteln sich die kleinen Abweichungen aus, das Profil ist flach. Die Verteilung des Schmutzlicht aus jeder Subapertur ist anders und wird bei der benutzten 8-Bit-Graustufenskala nicht mehr aufgelöst. 


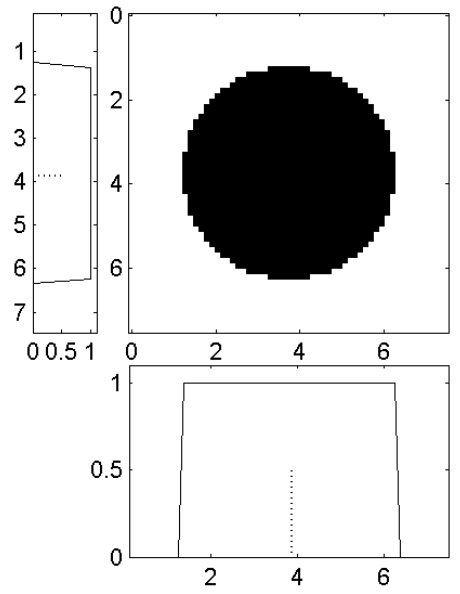

a

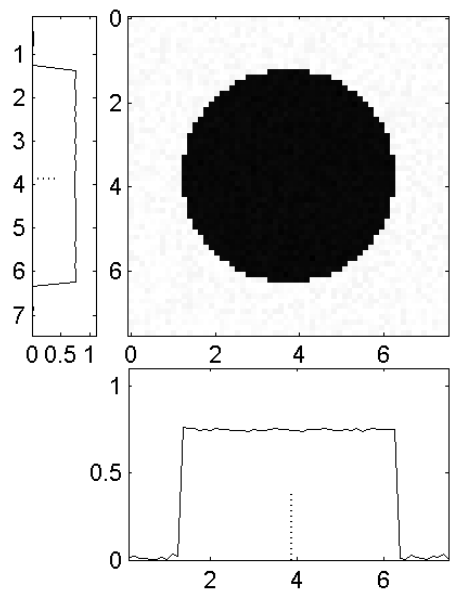

b

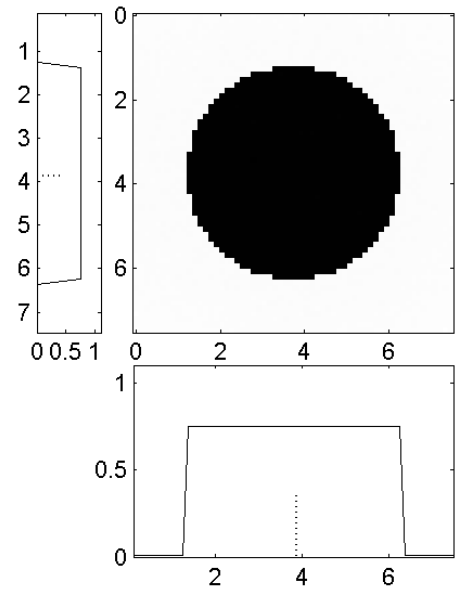

c

Abbildung 3.2: Vorgegebene Verteilung $\left|f^{0}\right|^{2}(\mathbf{a})$, Näherungslösung $\left|f^{P}\right|^{2}$ einer Subapertur aus IFQA (b), Überlagerung der 121 IFQA-Lösungen (c). Einfache Graustufenskala. Achsen in $\mathrm{mm}$.

\subsection{Experiment}

\subsubsection{Messung der Stufentiefen des DPE Nr. I}

Die Stufentiefen des DPE Nr. I sind nach der Herstellung mit einem interferenz-optischen Profilometer (Promap 512) vermessen worden. Es wurden Messungen in der Nähe der vier Ecken und in der Mitte des DPE durchgeführt. Die Ergebnisse sind in Tabelle (3.2) zusammengestellt.

\begin{tabular}{l|c|c|c|c} 
& 0. Stufe $[\mathrm{nm}]$ & 1. Stufe $[\mathrm{nm}]$ & 2. Stufe $[\mathrm{nm}]$ & 3. Stufe $[\mathrm{nm}]$ \\
\hline Position 1 & 0 & 128,5 & 248 & 379 \\
Position 2 & 0 & 128 & 251 & 375 \\
Position 3 & 0 & 126,5 & 250,5 & 379 \\
Position 4 & 0 & 129 & 257 & 381 \\
Position 5 & 0 & 125 & 244,5 & 372,5
\end{tabular}

Tabelle 3.2: Gemessene Stufentiefen des DPE Nr. I

Die Solltiefe für eine Stufe beträgt nach Gleichung (1.9) $D_{i}=\frac{248 \mathrm{~nm}}{4(1,51-1)}=122 \mathrm{~nm}$. Aus den Werten aus Tabelle (3.2) errechnet sich eine mittlere Stufentiefe $\bar{D}=126 \mathrm{~nm} \pm 3 \mathrm{~nm}$. Die Fehlergrenzen geben die Standardabweichung an. 


\subsubsection{Optische Rekonstruktion des DPE Nr. I mit HeNe-Laser}

Zur Charakterisierung der optischen Funktion des DPE Nr. I sollen zuerst die Ergebnisse der optischen Rekonstruktion mit der kohärenten Strahlung eines HeNe Lasers dargestellt werden. Die Entfernung $z_{1}$ der Signalebene vom DPE bei Verwendung der Wellenlänge $\lambda_{1}$ statt der Designwellenlänge $\lambda_{0}$ ergibt sich aus:

$$
z_{1}=\frac{\lambda_{0}}{\lambda_{1}} z_{0}=\frac{248 \mathrm{~nm}}{633 \mathrm{~nm}} 1250 \mathrm{~mm}=490 \mathrm{~mm} .
$$

Der vom DPE erzeugte Phasenhub ist für $\lambda=633 \mathrm{~nm}$ zu gering. Dies führt dazu, dass ein erheblicher Teil des HeNe-Strahls ungebeugt als nullte Ordnung weiterpropagiert. Die relativen Intensitäten innerhalb der ersten Beugungsordnung bleiben davon unbeeinflußt. Jede Subapertur definiert sich eine eigene optische Achse. Die Subaperturen am Rand des DPE erzeugen einen zur Mitte abgelenkten Strahl, der in der Signal-Ebene von ihrer nullten Ordnung räumlich getrennt ist. Somit kann die optische Rekonstruktion eines DPE bei einer von der Design-Wellenlänge abweichenden Wellenlänge ohne störende Einflüsse der nullten Ordnung untersucht werden.

In Abbildung (3.3) ist die optische Rekonstruktion mit einem HeNe-Laserstrahl in einer Entfernung $z_{1}=490 \mathrm{~mm}$ vom DPE Nr. I gezeigt. Die Rekonstruktion einer Subaper-

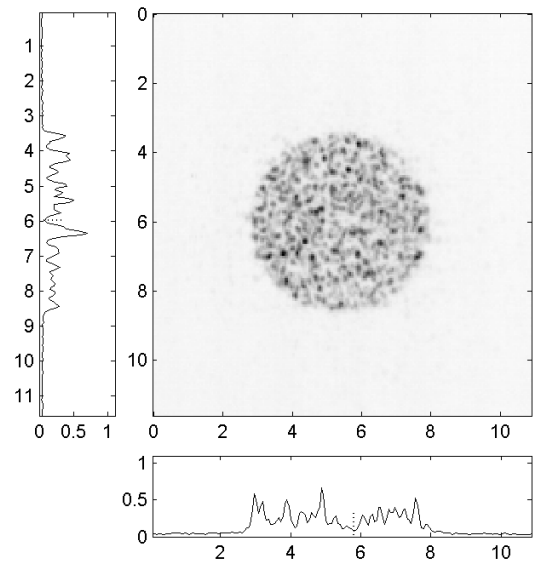

a

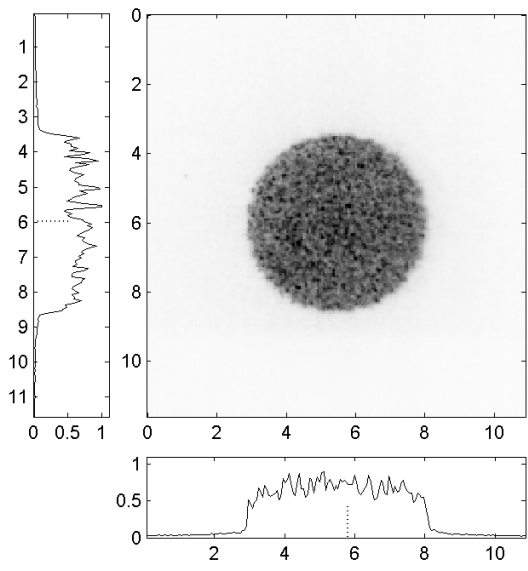

b

Abbildung 3.3: Optische Rekonstruktion des DPE Nr. I mit HeNe-Laser.

1 Subapertur (a), 32 Subaperturen (b).

Einfache Graustufenskala, Achsen in mm.

tur zeigt auf dem Träger des 5-mm-Spots ein durchmoduliertes Specklemuster. Diese Intensitätsfluktuationen sind auf Phasensingulariäten des Wellenfeldes in der Signalebene zurückzuführen [1]. Der IFTA/IFQA-Algorithmus behandelt die Phasenwerte benachbarter Pixel als unabhängige Variablen. Wenn die Phasenwerte kreisförmig benachbarter Pixel Werte aus dem gesamten Interval $[0,2 \pi]$ besitzen, z. B. eine „Phasenwendeltreppe“ 
mit Werten von 0 bis $2 \pi$, dann ist der Phasenwert in der Mitte nicht wohldefiniert. Solche Phasensingularitäten sind mit einer Nullstelle der Intensität des Wellenfeldes verbunden [1]. Intensitätseinbrüche treten auch bei Phasensprüngen zwischen benachbarten Pixeln von ungefähr $\pi$ auf. Die Form des Specklemusters hängt von den für die Signalebene gewählten Zufallszahlen der Anfangsphase $\phi^{0}$ (vgl. Abschnitt (1.4)) ab. Aus unterschiedlichen Anfangsphasen $\phi^{0}$ für dasselbe vorgegebene Objekt $\left|f^{0}\right|$ errechnet der IFTA/IFQAAlgorithmus DPE-Funktionen $\exp \left(i \psi_{\text {IFQA }}\right)$, die bei der optischen Rekonstruktion verschiedene Specklemuster erzeugen. Die Überlagerung verschiedener Specklemuster führt zu einer gewissen Ausmittelung der Intensitätsschwankungen. Abbildung (3.3)b zeigt die optische Rekonstruktion von 32 Subaperturen, deren Transmissionsfunktionen $\exp \left(i \psi_{\mathrm{IFQA}}\right)$ aus 32 verschiedenen Zufalls-Anfangsphasen $\phi^{0}$ berechnet wurden. Bei einer Subapertur liegt der Mittelwert der Intensität bei etwa 0,3 mit einer Schwankung von $\pm 100 \%$. Bei 32 Subaperturen liegt der Mittelwert der Intensität bei etwa 0,7 mit einer Schwankung von $\pm 25 \%$.

\subsubsection{Optische Rekonstruktion des DPE Nr. I mit KrF Excimer- Laser}

Bei der optischen Rekonstruktion mit der partiell-kohärenten Strahlung des L1000 Excimer Lasers tritt die im vorigen Abschnitt beschriebene Speckleproblematik in den Hintergrund. Als zentrales Problem erweist sich die passgenaue Überlagerung der Signale aus den einzelnen Subaperturen.

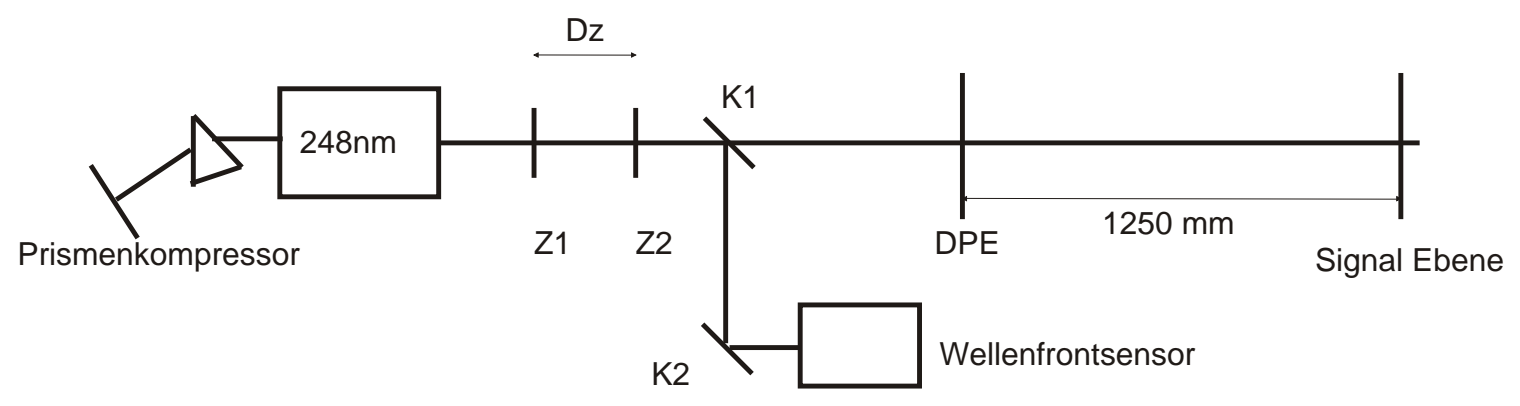

K1: Quarzkeil $6^{\circ}$

Z1: Zylinderlinse, $\mathrm{R}=52 \mathrm{~mm}$, kurze Achse

K2: Quarzkeil 6

Z2: Zylinderlinse, $\mathrm{R}=134 \mathrm{~mm}$, kurze Achse

DPE: Diffraktives Phasenelement $\quad$ Dz: Abstand zwischen Z1 und Z2

Abbildung 3.4: Experimenteller Aufbau DPE Nr. I

In Abbildung (3.4) ist der benutzte experimentelle Aufbau dargestellt. Die Rekonstruktion des DPE Nr. I wurde mit verschiedenen optischen Konfigurationen getestet:

1. Mit dem Rohstrahl des L1000. 
2. Mit einem Prismenkompressor [12] und einem auf Unendlich eingestelltem Zylinderteleskop in der kurzen Achse $(D z=352 \mathrm{~mm})$.

3. Mit dem Prismenkompressor und einem verstimmten Zylinderteleskop in der kurzen Achse $(D z=358 \mathrm{~mm})$, um die Wellenfrontcharakteristik der kurzen Achse zu glätten.

Durch den Prismenkompressor wird der Strahlquerschnitt in der langen Achse verringert, bevor der Strahl auf den Rückspiegel des Resonators trifft. Die hochdivergenten Anteile des Strahls werden nicht in den Resonator zurückgeführt. Divergenz des ausgekoppelten Strahls und Laserausgangsleistung nehmen ab [12]. Der Keil (K1) kann zur Messung der Wellenfrontcharakteristik in den Strahlengang gestellt werden. Der optische Weg von K1 bis zum Wellenfrontsensor entspricht der Strecke von K1 bis zum DPE, um die auf das DPE einfallende Wellenfrontcharakteristik zu messen. Die Oberfläche eines Quarzkeils reflektiert ca. $4 \%$ des Strahls. Die Verwendung der Keile ist notwendig, um den Strahl vor dem empfindlichen Sensor abzuschwächen, ohne die Wellenfront durch Propagation durch ein abschwächendes Dielektrikum zu verzerren.

In Abbildung (3.5) sind die Rekonstruktionen des DPE Nr. I bei den drei verschiedenen optischen Konfigurationen gezeigt:

1. Rohstrahl: Der Strahlquerschnitt des Rohstrahls betrug ca. $28 \mathrm{~mm} \cdot 12 \mathrm{~mm}$. Die Wellenfront des Rohstrahls ist stark gekrümmt. Die maximale Auswölbung der Wellenfront beträgt 144 Wellenlängen. In der langen Achse beträgt die Divergenz der Hauptstrahlrichtungen 7,3 mrad, in der kurzen Achse 3,0 mrad. Das Spot-Signal aus einer Subapertur ist aufgrund der geringen Kohärenz stark verwaschen und ohne scharfe Konturen. Speckleartige Intensitätsfluktuationen treten nicht auf.

2. Prismenkompressor, Z1, Z2, Dz=352 mm: Die Verwendung des Prismenkompressors in der langen Achse verringert die Divergenz der Hauptstrahlrichtungen von 7,3 mrad auf 2,0 mrad. Das auf Unendlich justierte Zylinderteleskop weitet den Strahl um einen Faktor von ca. 2,5 auf. Damit ist die Ausleuchtung des gesamten DPE mit seiner Ausdehnung von 27, $4 \mathrm{~mm} \cdot 27,4 \mathrm{~mm}$ möglich. Die Divergenz in der kurzen Achse reduziert sich dadurch leicht von 3,0 mrad auf 2,3 mrad. Die maximale Auswölbung der Wellenfront beträgt 56 Wellenlängen. Die Rekonstruktion einer Subapertur ist in der langen Achse aufgrund der erhöhten Kohärenz durch Verwendung des Prismenkompressors erheblich verbessert. In der kurzen Achse bewirkt die Aufweitung ebenfalls eine Zunahme der räumlichen Kohärenz [31], und ein schwaches Specklemuster entsteht in dieser Achse. Bei der Rekonstruktion aus 121 Subaperturen ist die Flankensteilheit jedoch reduziert durch die nicht-deckungsgleiche Überlagerung (vgl. Abbildung (3.6)) der einzelnen Spots.

3. Prismenkompressor, Z1, Z2, Dz=358 mm: Die der Wellenfront angepasste Verstimmung des Zylinderteleskops ermöglicht die fast vollständige Korrektur der Divergenz der Hauptstrahlrichtungen in der kurzen Achse. Die maximale Auswölbung 


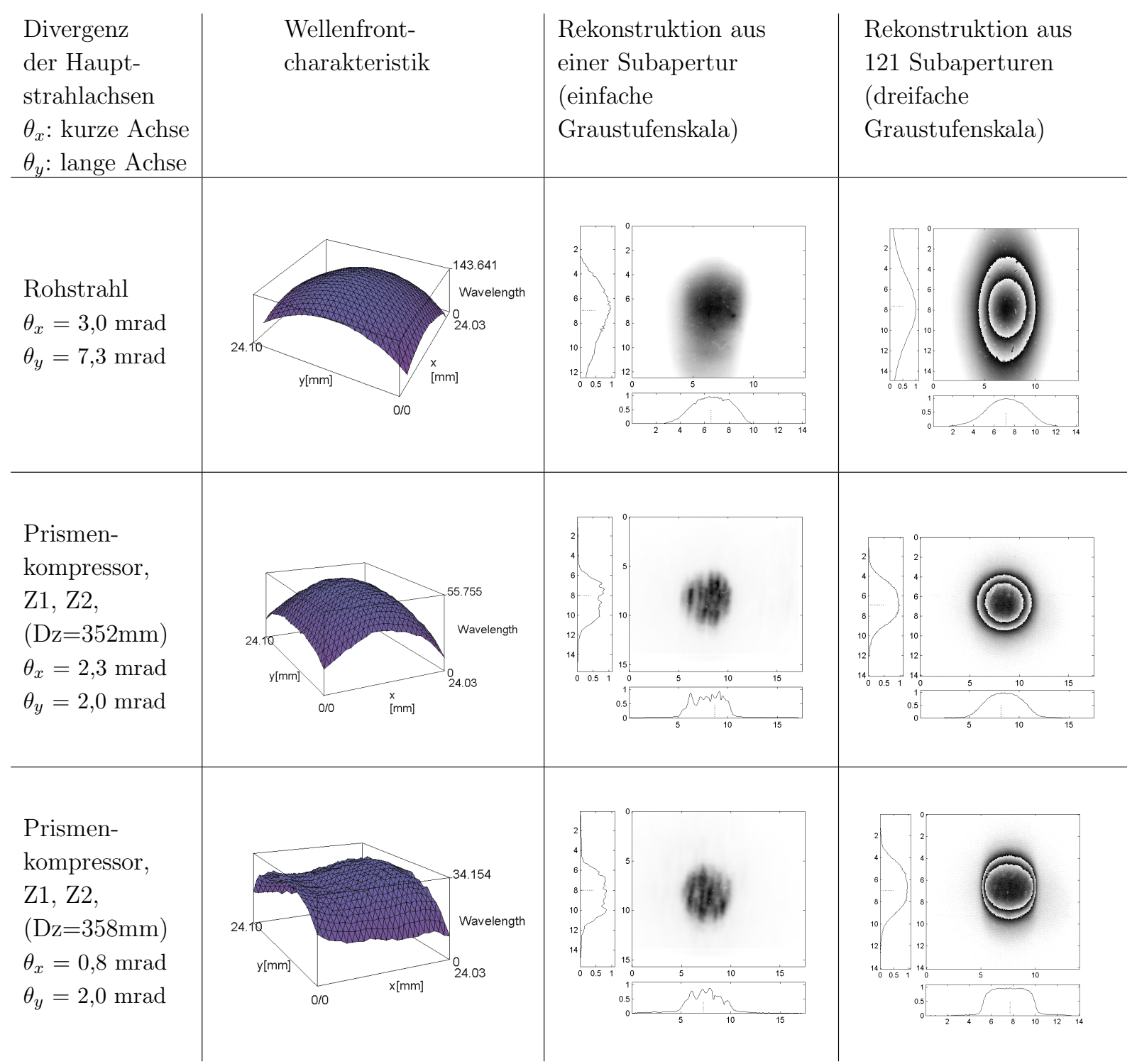

Abbildung 3.5: Optische Rekonstruktion DPE Nr. I mit KrF Excimer-Laser. Achsen in mm.

der Wellenfront beträgt 34 Wellenlängen. Die Rekonstruktion aus einer Subapertur ist davon unabhängig und deren Rekonstruktion entspricht der Rekonstruktion mit dem Prismenkompressor bei Dz=352 mm. Die Überlagerung der 121 Spots aus den einzelnen Subaperturen gelingt bezüglich der kurzen Achse deutlich besser aufgrund der nahezu parallelen Hauptstrahlrichtungen. In der kurzen Achse erzeugt dies steile Kanten, und das entsprechende Schnittprofil kann mit einem Super-Gauss-Profil der Ordnung $n=9$ angefittet werden. Für die lange Achse ergibt sich $n=3$. 


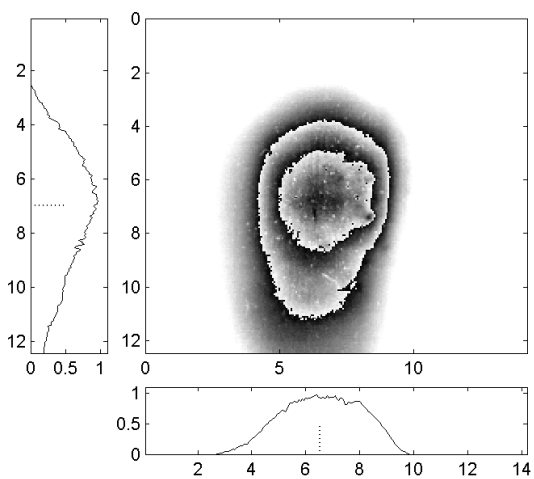

a

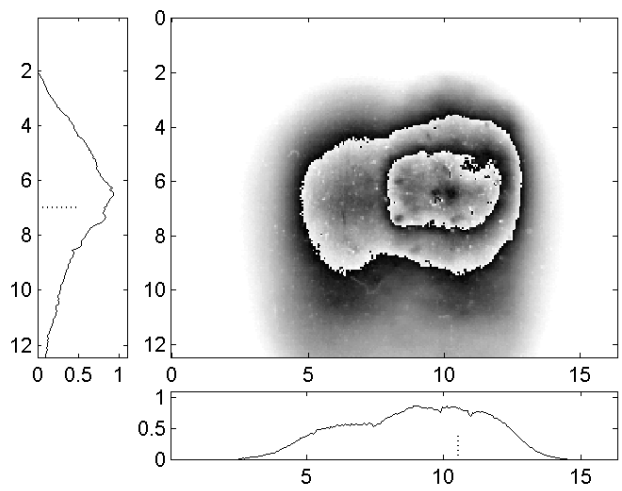

b

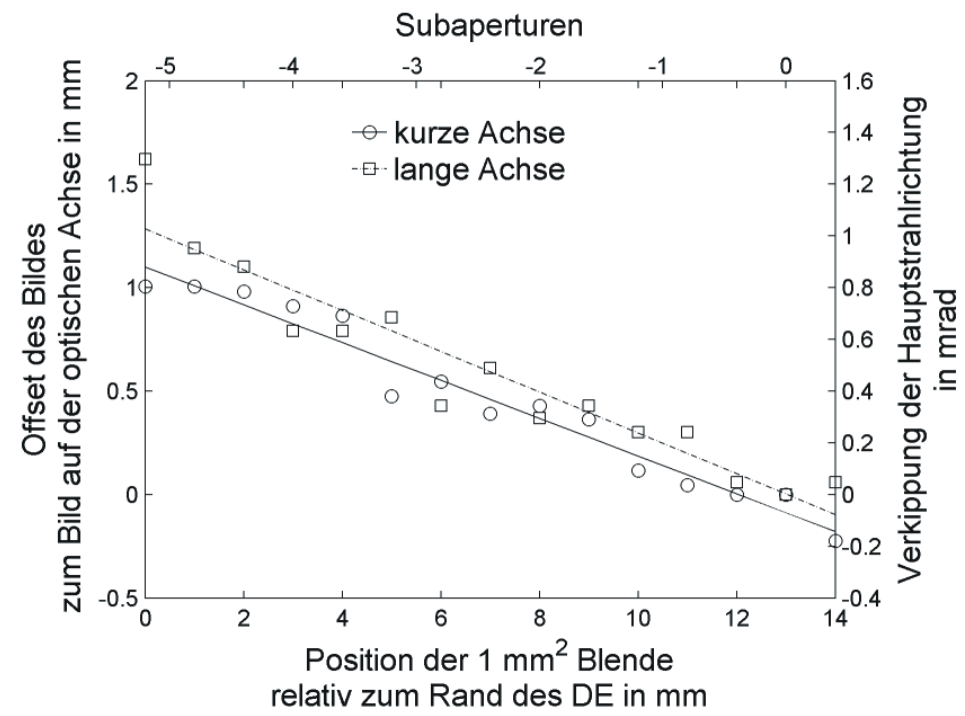

C

Abbildung 3.6: a,b: Dreifache Graustufenskala, Achsen in mm.

a: Optische Rekonstruktion der Subapertur $(5,-5)$ mit dem KrF Rohstrahl.

b: Optische Rekonstruktion der Subaperturen $(5,-5)$ und $(5,1)$ mit dem KrF Rohstrahl. Die Signale der beiden Subaperturen überlagern nicht deckungsgleich.

c: Quantitative Messung des räumlichen Versatzes der Signale aus verschiedenen Subaperturen (Prismenkompressor, Z1, Z2, Dz = $352 \mathrm{~mm})$

In der Abbildung (3.6)a ist die Rekonstruktion der Subapertur (5,-5) mit dem Rohstrahl gezeigt. In der Abbildung (3.6)b ist die Rekonstruktion der Subaperturen $(5,-5)$ und $(5,1)$ 
mit dem Rohstrahl gezeigt. Die beiden Signale überlagern nicht deckungsgleich trotz der Berücksichtigung des geometrischen Subaperturversatzes (vgl. Gleichung (3.1)). Die Apertur $(5,-5)$ aus der äußersten Ecke des DPE erzeugt das schwächere, linke Bild, die Apertur $(5,1)$ das rechte Bild. Der Versatz der erzeugten Spot-Bilder in Abhängigkeit der beleuchteten Subapertur wurde an der optischen Konfiguration Nr.2 (Prismenkompressor, Z1, $\mathrm{Z} 2, D z=352 \mathrm{~mm}$ ) quantitativ ausgemessen und ist in der Abbildung (3.6)c dargestellt. Das DPE Nr. I wurde abgeschattet bis auf eine $1 \mathrm{~mm}^{2}$ große Apertur, die in Schritten von $1 \mathrm{~mm}$ vom Rand zur Mitte des DPE verschoben wurde. An jeder Position ist das rekonstruierte Spot-Bild mit der CCD-Kamera aufgenommen worden. Die Verschiebung des Schwerpunktes der Spot-Bilder relativ zu dem Schwerpunkt des Spot-Bildes aus der Apertur auf der optischen Hauptachse ist auf der y-Achse aufgetragen. Die rechte y-Achsenbeschriftung ist eine Umrechnung dieser Daten ins Winkelmaß (mrad). Die Messung wurde für die lange und die kurze Laserachse durchgeführt.

Die relative Verkippung der Hauptstrahlachsen vom Rand zur Mitte des DPE Nr. I beträgt für beide Achsen ca. 1 mrad. Extrapoliert man dieses Ergebnis auf die relative Verkippung von einem Rand zum gegenüberliegenden Rand des DPE ergeben sich ca. 2 mrad. Dies entspricht recht genau den Werten für die Divergenz der Hautpstrahlachsen über die Gesamtapertur des DPE aus der Wellenfrontmessung von 2,0 mrad für die lange Achse und 2,3 mrad für die kurze Achse (vgl. Abbildung (3.5)).

\subsubsection{Effizienzmessung von DPE Nr. I}

Die Transmission, Beugungs- und Systemeffizienz ist durch Pulsenergiemessungen mit einem Joulemeter bestimmt worden. In Abbildung (3.7) ist der entsprechende Aufbau skizziert. Alle Messungen sind über 20-30 Pulse gemittelt worden.

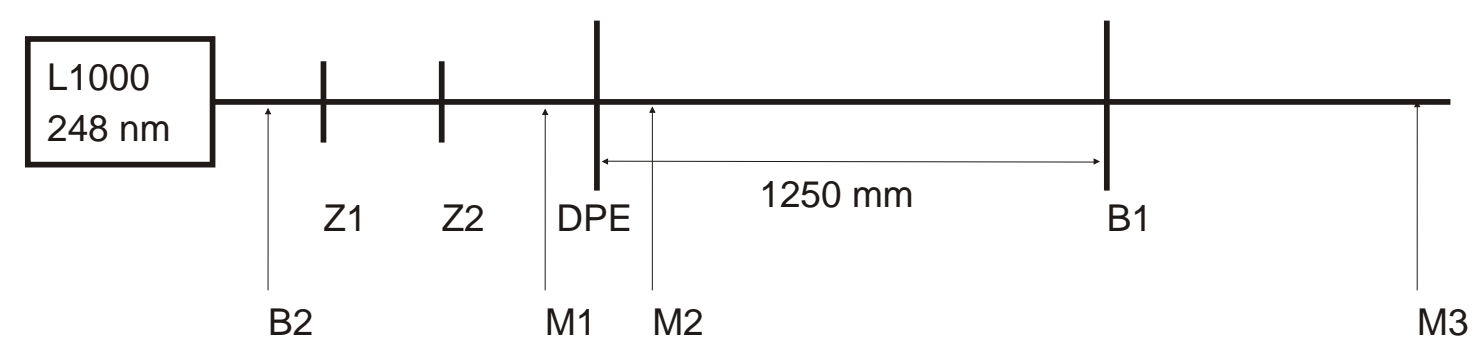

Z1: Zylinderlinse 1

Z2: Zylinderlinse 2

M1-M3: Messung 1-3
B1: kreisförmige Lochblende $8 \mathrm{~mm}$ Durchmesser

B2: $9 \mathrm{~mm}$ Blende, um Überstrahlung des DPE zu vermeiden

Abbildung 3.7: Experimenteller Aufbau zur Effizienzmessung, DPE Nr. I 
Die Energie im Nahfeld des Rohstrahls betrug 394 mJ. Die Energie im Nahfeld mit Prismenkompressor $262 \mathrm{~mJ}$. Der Energieverlust durch den Prismenkompressor beträgt also $1-262 \mathrm{~mJ} / 394 \mathrm{~mJ}=34 \%$. In der folgenden Tabelle sind die Messwerte M1-M3 (vgl. Abb. (3.7)) zusammengestellt.

\begin{tabular}{c|c|c|c} 
Ohne B2 & Transmission & Systemeffizienz & Beugungseffizienz $\eta$ \\
\hline M1 $=188 \mathrm{~mJ}$ & & & \\
$\mathrm{M} 2=143 \mathrm{~mJ}$ & $143 / 188=76 \%$ & & \\
$\mathrm{M} 3=102 \mathrm{~mJ}$ & & $102 / 188=54 \%$ & $102 / 143=71 \%$ \\
\hline Mit B2 & & & \\
\hline $\mathrm{M} 1=22,7 \mathrm{~mJ}$ & & & \\
$\mathrm{M} 2=20,3 \mathrm{~mJ}$ & $20,3 / 22,7=89 \%$ & & $14,4 / 20,3=71 \%$ \\
$\mathrm{M} 3=14,4 \mathrm{~mJ}$ & & $14,4 / 22,7=63 \%$
\end{tabular}

Bei der Messung mit der Blende B2 beträgt die Transmission 89\%, das entspricht dem Verlust durch Reflexion an den beiden unbeschichteten Quarzglasoberfächen des DPE von 5-6\% pro Oberfläche. Ohne die Blende B2 werden die hochdivergenten äußeren Strahlanteile durch die umrandende Chrombeschichtung des DPE abgeschattet. Dies erklärt den geringeren Wert von $76 \%$ für die Transmission ohne Blende B2.

Die Messung M3 wurde nicht direkt sondern ca. 300 mm hinter der Blende B1 ausgeführt, um den Energiemesskopf durch die hohen Fluenzen in der fokalen Signal-Ebene nicht zu zerstören. Die Beugungseffizienz ist unabhängig davon, ob das DPE überstrahlt wird und beträgt in beiden Fällen $71 \%$.

Die Systemeffizienz ist definiert als der Anteil der auf das DPE eingestrahlten Energie, der in die gewünschten Geometrie einfällt. Mit der Blende B2 beträgt die Systemeffizienz $63 \%$ und ohne B2 54\%.

\subsection{Ergebnis}

Das Strahlformungsergebnis einer Subapertur bei Beleuchtung des DPE Nr. I mit einem hochkohärenten HeNe-Laserstrahl (Abb. (3.3)a) weicht signifikant von dem berechneten Ergebnis des IFTA/IFQA-Algorithmus ab (Abb. (3.2)b). Die unerwünschten Intensitätsfluktuationen („Speckles“) sind auf Phasensingularitäten der rekonstruierten Signalwelle zurückzuführen [1]. Die Modellierung der Phasenwerte benachbarter Pixel als unabhängige Variablen durch den IFTA/IFQA-Algorithmus ist für diese Intensitätsfluktuationen verantwortlich. Für einfache Geometrien bieten sich DPE-Designkonzepte an, die die Strahlpropagation mittels geometrischer Optik modellieren [15], [16]. Bei diesen DesignKonzepten wird eine Signalwelle mit einer stetigen Phasenfunktion erzeugt und somit die Entehung der Speckles unterdrückt.

Der Mittelungseffekt durch die Überlagerung der Signalwellen aus vielen Subaperturen kann die Intensitätseinbrüche deutlich reduzieren (Abb. (3.3)b). Diese Rekonstruktion 
aus 32 Subaperturen zeigt, dass eine deckungsgleiche Überlagerung der einzelnen 5mmSpots mit der ebenen Wellenfrontcharakteristik des HeNe-Lasers möglich ist, und der in Abschnitt (3.3.3) beschriebene Versatz dieser einzelnen 5mm-Spots bei Beleuchtung des DPE mit dem L1000-Excimer-Laserstrahl auf dessen gekrümmte Wellenfrontcharakteristik zuruckzuführen ist.

Die optische Rekonstruktion aus einer Subapertur mit dem Rohstrahl des L1000 zeigt keine Speckles mehr, jedoch ist das erhaltene Signal weder rund noch homogen. Die Verbesserung der räumlichen Kohärenz durch den Einsatz eines Prismenkompressors erzeugte eine annähernd runde Spot-Grundfläche mit definierten Kanten. Die Korrektur der Wellenfrontcharakteristik durch Verwendung eines angepassten Zylinderteleskops ermöglicht die passgenaue Überlagerung der einzelnen 5mm-Spots. Diese beiden Modifikationen am Rohstrahl des L1000 führen zu einem homogenen Profil mit steilen Kanten in der Achse des Zylinderteleskops (Abb. (3.5)).

Die gemessene Beugungseffizienz $\eta(\exp )=71 \%$ liegt über der Abschätzung $\eta(Z)=61 \%$ (Tabelle (3.1)). Durch die Verwendung einer $8 \mathrm{~mm}$ kreisförmigen Blende statt einer 5mmBlende sind Anteile in den Flanken mitgemessen worden, die nicht zur Grundfläche des 5mm-Spots gehören. Die Messgenauigkeit der Energiemessköpfe und die Schwankungen der Laserparameter liegen im Prozentbereich. Darüber hinaus ist $\eta(Z)$ nur ein theoretischer Anhaltspunkt für die Effizienz, da es keinen analytischen Ausdruck zur Ermittlung der theoretischen Beugungseffizienz eines zweidimensionalen DPE gibt (vgl. Abschnitt $(1.3))$. 


\section{Korrektur der divergenten Wellenfrontcharakteristik}

Im vorigen Kapitel 3 ist die Formung des L1000-Laserstrahls in einen runden 5mm-Spot durch ein DPE beschrieben worden. Mit dem Rohstrahl ist das Ergebnis unbefriedigend. Bei Verwendung des Prismenkompressors ist die Strahlformung in einer Subapertur zufriedenstellend, die Spots aus verschiedenen Subaperturen überlagern sich jedoch aufgrund der divergenten Hauptstrahlachsen nicht deckungsgleich. Die unterschiedlichen Hauptstrahlachsen an den Mittelpunkten der Subaperturen sind in weiteren DPE-Designs berücksichtigt worden. Die Korrektur der Wellenfrontcharakteristik wird somit in das DPE eingerechnet, anstatt die Wellenfront durch Verwendung eines Teleskops zu korrigieren.

\subsection{Design und Berechnung der DPE Nr. II/III}

Die vorgegebene Signalverteilung $\left|f^{0}\right|^{2}$ für DPE Nr. II ist wiederum ein runder, homogener Spot mit $5 \mathrm{~mm}$ Durchmesser. Das DPE Nr. III soll vier solcher Spots erzeugen (vgl. Abbildung (4.1)). Die optische Achse befindet sich jeweils in der Mitte des dargestellten quadratischen Ausschnitts.
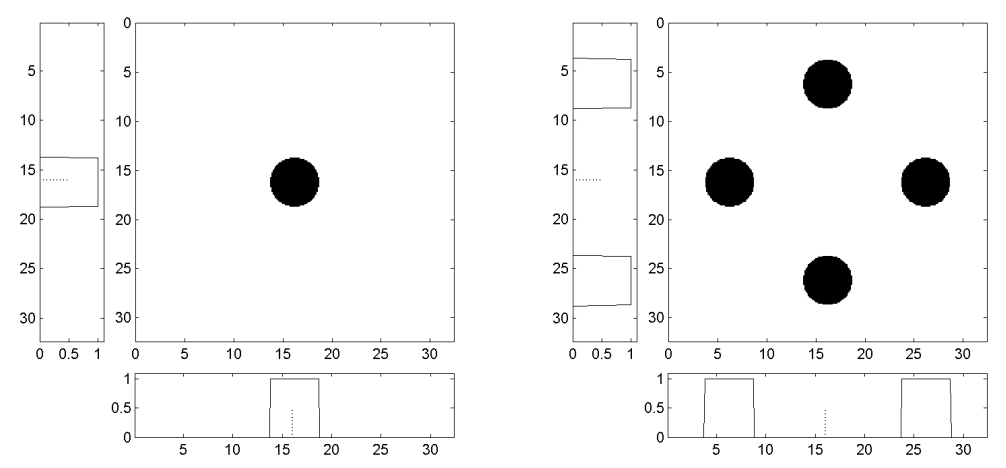

Abbildung 4.1: Vorgegebene Intensität $\left|f^{0}\right|^{2}$ für DPE Nr. II (links) und DPE Nr. III (rechts). Achsen in $\mathrm{mm}$.

Die beiden DPE sind berechnet worden zur Bestrahlung mit dem durch den Prismenkompressor modifizierten Strahl des L1000 ohne Verwendung eines Teleskops. Die Wellenfrontcharakteristik aus einer Shack-Hartmann Messung in dieser Konfiguration (vgl. Abbildung (4.2)a,b) kann auf einer Strahlquerschnittsfläche von ca. $28 \cdot 18 \mathrm{~mm}^{2}$ ausgewertet werden. Die DPE sind daher aus 11 7 Subaperturen zusammengesetzt, deren Ausdehnung jeweils $2,49 \cdot 2,49 \mathrm{~mm}^{2}$ beträgt. 


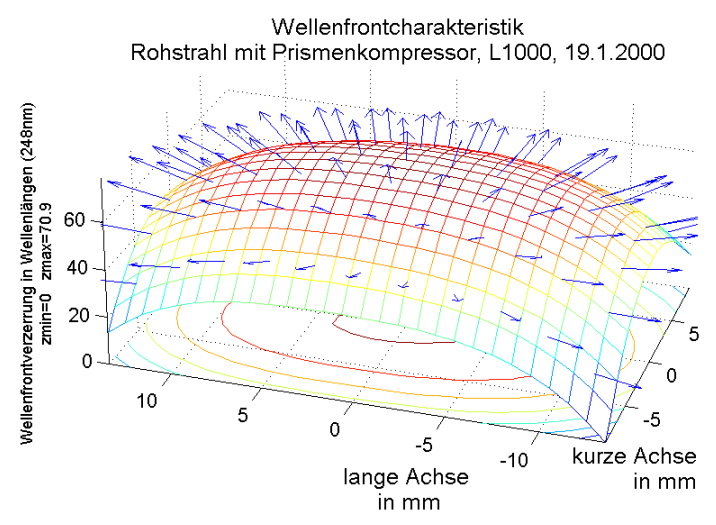

a

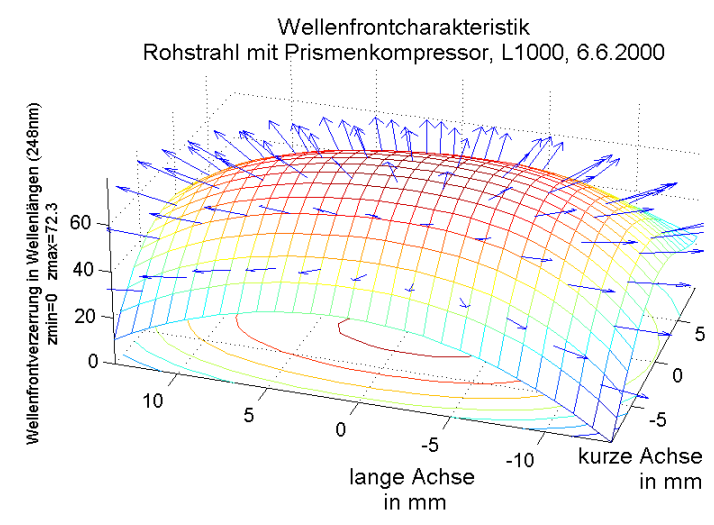

b

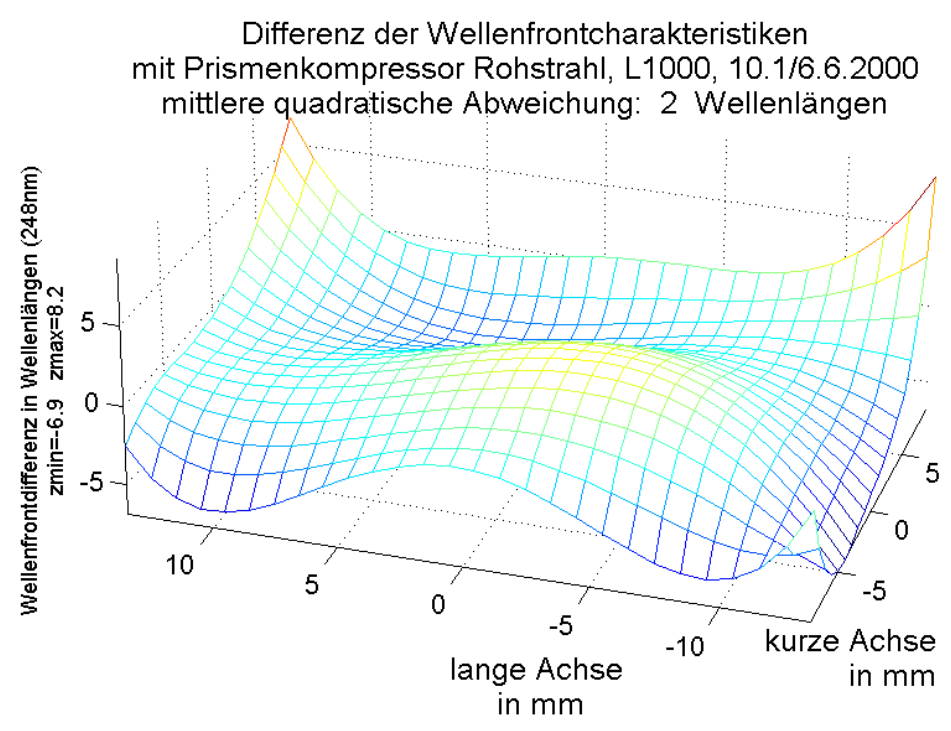

c

Abbildung 4.2: Normalenvektoren und Reproduzierbarkeit der Wellenfrontcharakteristik. Zwei verschiedene Messungen im Abstand von 5 Monaten (a) und (b). Ihre Differenz ist in (c) gezeigt. Die mittlere quadratische Abweichung der beiden Messungen beträgt 2,0 Wellenlängen, das entspricht 2, $8 \%$ der maximalen Auswölbung von 72,3 Wellenlängen.

Die auf das DPE einfallende Wellenfront wird als aus stückweise ebenen Wellenfronten zusammengesetzt modelliert. Die Richtung der Normalenvektoren $\mathbf{n}_{i j}$ der auf jede 
Subapertur $(i, j)$ einfallenden ebenen Wellenfront ist durch die in Abbildung ((4.2)a, $\mathbf{a})$ auf der Wellenfrontcharakteristik senkrecht stehenden Vektoren gegeben. In den IFQARechnungen für die DPE Nr. II/III ist für jede Subapertur ein Phasenfaktor eingerechnet worden, um diese Verkippungen zu kompensieren.

Sei $\mathbf{K}_{i j}=\left(\begin{array}{l}i \\ j\end{array}\right)$ der Koordinatenvektor der Subapertur in der i. Zeile und der j. Spalte, und $\hat{\mathbf{K}}_{i j}$ der entsprechende Einheitsvektor (vgl. Abschnitt 3.2). Das Beugungsbild $f$ in der Signalebene muss dann um den geometrischen Versatz jeder Subapertur $\left\langle\mathbf{K}_{i j}, \Delta \mathbf{u}\right\rangle$ und den Versatz des Signals aufgrund der geneigten Wellenfront zurück verschoben werden. Sei $\alpha$ der Winkel zwischen dem Wellenfront-Normalenvektor

$$
\mathbf{n}_{i j}=\left(\begin{array}{c}
\mathrm{nx}_{i j} \\
\mathrm{ny}_{i j} \\
\mathrm{nz}_{i j}
\end{array}\right)
$$

und dem Einheitsvektor in Richtung der optischen Achse, dann gilt:

$$
\mathbf{x}^{\prime} \mathbf{v}_{i j}=\left\langle\mathbf{K}_{i j}, \Delta \mathbf{u}\right\rangle \hat{\mathbf{K}}_{i j}+\left(\tan (\alpha) \cdot z_{0}\right)\left(\begin{array}{c}
\mathrm{nx}_{i j} \\
\mathrm{ny}_{i j}
\end{array}\right) \in \mathbb{R}^{2}
$$

In den quadratischen Phasenfaktor $W\left(z_{0}, \mathbf{u}-\mathbf{x}_{\mathbf{v}}^{\prime}\right)^{*}$ der IFQA-Rechnung (vgl. Abschnitt (1.4.2)) muss der Vektor $\mathbf{x}_{\mathbf{v}_{i j}}^{\prime}$ aus Gleichung (4.1) eingesetzt werden.

Um die Stabilität und Reproduzierbarkeit solcher Wellenfrontcharakteristiken beurteilen zu können, sind wiederholt Shack-Hartmann Messungen durchgeführt worden. Zwischen diesen Messungen sind Lasergas und Rückspiegel ausgetauscht worden. Abbildung (4.2) zeigt zwei Messungen in einem Abstand von 5 Monaten. Die mittlere quadratische Abweichung der beiden Wellenfrontcharakteristiken beträgt nur 2,8 \%.

Die übrigen IFTA- und IFQA-Parameter sind zur Vergleichbarkeit mit DPE Nr. I so wie in Abschnitt (3.2) gewählt:

\begin{tabular}{lc|lrl}
\multicolumn{2}{l|l}{ IFTA-Parameter DPE Nr. II/III } & IFQA-Parameter DPE Nr. II/III & \\
\hline Pixelzahl N (vert.) & 996 & Pixelgröße DPE $\delta u$ & 2,5 & $\mu \mathrm{m}$ \\
Pixelzahl M (hor.) & 996 & Subaperturgröße $\Delta u$ & 2,49 & $\mathrm{~mm}$ \\
max. Iterationszahl & 110 & Übertragungslänge $z_{0}$ & 1,25 & $\mathrm{~m}$ \\
Abbruchkriterium $\mathrm{rms}_{s}$ & $10^{-5}$ & Pixelgröße Signal $\delta x$ & 124,5 & $\mu \mathrm{m}$ \\
& & Iterationszahl P & 45 & \\
& & Stufenzahl $Z$ & 4 &
\end{tabular}

Analog zu DPE Nr. I aus Abschnitt (3.2) ergeben sich aus den IFTA/IFQA-Rechnungen die in Tabelle (4.1) angegebenen Werte für die Effizienz $\eta$ und den Objektfehler $r m s_{s}$. Die Werte für Effizienz und $r m s_{s}$ des DPE Nr. II sind gleich denen von DPE Nr. I (vgl. Tabelle (3.1)). Für die kompliziertere Beugungsstruktur von DPE Nr. III ist die Effizienz um einige Prozentpunkte erniedrigt und der Objektfehler erhöht. 


\begin{tabular}{c|c|c|l|c|c|l|} 
& \multicolumn{3}{|c|}{ DPE Nr. II } & \multicolumn{3}{c|}{ DPE Nr. III } \\
& IFTA & IFQA & & IFTA & IFQA & \\
\hline$r m s_{s}$ & $10^{-5}$ & $3 \cdot 10^{-3}$ & & $10^{-5}$ & $5 \cdot 10^{-3}$ & \\
$\eta$ & $92 \%$ & $75 \%$ & $\eta(Z)=61 \%$ & $88 \%$ & $71 \%$ & $\eta(Z)=58 \%$
\end{tabular}

Tabelle 4.1: Über 77 Subaperturen gemittelte Werte für Objektfehler $r m s_{s}$ und Effizienz $\eta$.

\subsection{Experiment}

Die optische Rekonstruktion aus einer Subapertur der DPE Nr. II/III mit dem HeNe Laser ergibt qualitativ ähnliche Resultate wie bei DPE Nr. I (vgl. Abbildung (3.3)a). Bei Beleuchtung mehrerer Subaperturen mit dem HeNe Laser überlagern die Spots nicht deckungsgleich wie bei DPE Nr. I (vgl. Abbildung (3.3)b), da die ebene Wellenfrontcharakteristik des HeNe Lasers nicht der in DPE Nr. II/III eingerechneten Wellenfront entspricht.

\subsubsection{Messung der Stufentiefen der DPE Nr. II/III}

Die Stufentiefen der DPE Nr. II/III sind nach der Herstellung mit einem interferenzoptischen Profilometer (Promap 512) vermessen worden. Es wurden an jedem DPE drei Messungen an Stellen mit unterschiedlicher lateraler Strukturgröße durchgeführt. Die Ergebnisse sind in Tabelle (4.2) zusammengestellt. Es zeigt sich, dass keine signifikante Abhängigkeit der Stufentiefe von der lateralen Strukturgröße besteht.

Die Solltiefe für jede Stufe beträgt nach Gleichung (1.9) $D_{i}=\frac{248 \mathrm{~nm}}{4(1,51-1)}=122 \mathrm{~nm}$. Aus den Werten der Tabelle (4.2) errechnet sich eine mittlere Stufentiefe $\bar{D}$ aus der Summe der drei Stufentiefen an den drei Messpunkten:

$$
\begin{aligned}
\bar{D} & =\sum_{i=1}^{9} D_{i} / 9 \\
& =127 \mathrm{~nm} \pm 15 \mathrm{~nm} \quad \text { für DPE Nr. II } \\
& =120 \mathrm{~nm} \pm 9 \mathrm{~nm} \quad \text { für DPE Nr. III }
\end{aligned}
$$

Die Fehlergrenzen geben die Standardabweichung an.

Um ein vierstufiges DPE herzustellen, sind zwei Ätzvorgänge notwendig (vgl. Abb. (1.8)). In einem Schritt wird die $\pi$-Phasenstufe geätzt mit einer Solltiefe von $244 \mathrm{~nm}$, im zweiten Ätzschritt die $\pi / 2$-Phasenstufe mit einer Solltiefe von $122 \mathrm{~nm}$. Wird ein DPE-Pixel in beiden Ätzschritten bearbeitet entsteht die $3 \pi / 2$-Phasenstufe mit einer Solltiefe von 366 nm. Aus den Werten der Tabelle (4.2) lassen sich die Ätztiefen $D_{i}^{\pi}$ und $D_{i}^{\pi / 2}$ für das Ätzen 


\begin{tabular}{|c|c|c|c|c|c|}
\hline & & $\begin{array}{c}\text { 0. Stufe } \\
{[\mathrm{nm}]}\end{array}$ & $\begin{array}{l}\text { 1. Stufe } \\
{[\mathrm{nm}]}\end{array}$ & $\begin{array}{l}\text { 2. Stufe } \\
{[\mathrm{nm}]}\end{array}$ & $\begin{array}{c}\text { 3. Stufe } \\
{[\mathrm{nm}]}\end{array}$ \\
\hline \multirow{9}{*}{ DPE Nr. II } & \multicolumn{5}{|l|}{ grobe Struktur } \\
\hline & \multirow{2}{*}{$\begin{array}{l}\text { Stufenabstand } D_{i} \\
\text { summierte Stufentiefe }\end{array}$} & 0 & 115 & 150 & 117 \\
\hline & & 0 & 115 & 265 & 382 \\
\hline & \multicolumn{5}{|l|}{ mittlere Struktur } \\
\hline & \multirow{2}{*}{$\begin{array}{l}\text { Stufenabstand } D_{i} \\
\text { summierte Stufentiefe }\end{array}$} & 0 & 119 & 145 & 121 \\
\hline & & 0 & 119 & 264 & 385 \\
\hline & \multicolumn{5}{|l|}{ kleine Struktur } \\
\hline & \multirow{2}{*}{$\begin{array}{l}\text { Stufenabstand } D_{i} \\
\text { summierte Stufentiefe }\end{array}$} & 0 & 115 & 148 & 116 \\
\hline & & 0 & 115 & 263 & 379 \\
\hline \multirow{9}{*}{ DPE Nr. III } & \multicolumn{5}{|l|}{ grobe Struktur } \\
\hline & \multirow{2}{*}{$\begin{array}{l}\text { Stufenabstand } D_{i} \\
\text { summierte Stufentiefe }\end{array}$} & 0 & 116 & 136 & 113 \\
\hline & & 0 & 116 & 252 & 365 \\
\hline & \multicolumn{5}{|l|}{ mittlere Struktur } \\
\hline & \multirow{2}{*}{$\begin{array}{l}\text { Stufenabstand } D_{i} \\
\text { summierte Stufentiefe }\end{array}$} & 0 & 113 & 130 & 112 \\
\hline & & 0 & 113 & 243 & 355 \\
\hline & \multicolumn{5}{|l|}{ kleine Struktur } \\
\hline & \multirow{2}{*}{$\begin{array}{l}\text { Stufenabstand } D_{i} \\
\text { summierte Stufentiefe }\end{array}$} & 0 & 112 & 134 & 116 \\
\hline & & 0 & 112 & 246 & 362 \\
\hline
\end{tabular}

Tabelle 4.2: Gemessene Stufentiefen der DPE Nr. II/III

der $\pi$ - und $\pi / 2$-Phasenstufen durch

$$
\begin{aligned}
& D^{\pi}=D_{1}+D_{2} \quad \text { und } \quad D^{\pi}=D_{2}+D_{3} \text {, sowie } \\
& D^{\pi / 2}=D_{1} \quad \text { und } \quad D^{\pi / 2}=D_{3}
\end{aligned}
$$

berechnen. Die Berechnung der Mittelwerte $\overline{D^{\pi}}$ und $\overline{D^{\pi / 2}}$ erfolgt analog zu Gleichung (4.2). Die Ergebnisse sind in Tabelle (4.3) zusammengestellt.

Die mittleren Stufentiefen der DPE Nr. II/III sind vergleichbar mit dem Wert für DPE Nr. I: $\bar{D}=126 \mathrm{~nm} \pm 3 \mathrm{~nm}$ (vgl. Tabelle (3.2)). Die Standardabweichung hingegen ist für die DPE Nr. II/III mit $\pm 15 \mathrm{~nm}$, bzw. $\pm 9 \mathrm{~nm}$, erheblich größer. Dies ist nicht auf einen ungleichmäßigen Ätzprozess zurückzuführen, sondern auf die entgegengesetzten Abweichungen von den Solltiefen für die $\pi$ - bzw. $\pi / 2$-Phasenstufe von $(+21 \mathrm{~nm},-5 \mathrm{~nm})$ für DPE Nr. II und $(+3 \mathrm{~nm},-8 \mathrm{~nm})$ für DPE Nr. III. Die Standardaweichungen für die $\pi-$ bzw. $\pi / 2$-Phasenstufen liegen im Bereich 1,5 nm bis $4 \mathrm{~nm}$ und definieren die erreichbare Gleichmäßigkeit der Ätztiefe über die laterale Ausdehnung der DPE bei einem Ätzvorgang. Das bedeutet, der kritische Parameter bei der Herstellung ist die Verweildauer in der Trockenätzanlage, der die Ätztiefe eines Ätzvorgangs bestimmt. 


\begin{tabular}{l|c|c|} 
& DPE Nr. II & DPE Nr. III \\
\hline mittlere Stufentiefe $\bar{D}[\mathrm{~nm}]$ & $\begin{array}{c}127 \pm 15 \\
+5 \mathrm{~nm}\end{array}$ & $\begin{array}{c}120 \pm 9 \\
-2 \mathrm{~nm}\end{array}$ \\
Abweichung vom Soll $D=122 \mathrm{~nm}$ & $\begin{array}{c}265 \pm 1,5 \\
+21 \mathrm{~nm}\end{array}$ & $\begin{array}{c}247 \pm 4 \\
+3 \mathrm{~nm}\end{array}$ \\
\hline mittlere Ätztiefe $\overline{D^{\pi}}[\mathrm{nm}]$ & $\begin{array}{c}117 \pm 2,5 \\
\text { Abweichung vom Soll } D^{\pi}=244 \mathrm{~nm}\end{array}$ & $\begin{array}{c}114 \pm 2 \\
-8 \mathrm{~nm}\end{array}$
\end{tabular}

Tabelle 4.3: Mittlere Ätztiefen mit Standardabweichung und deren Abweichung von den Solltiefen für DPE Nr. II/III.

\subsubsection{Optische Rekonstruktion der DPE $\mathrm{Nr}$. II/III mit $\mathrm{KrF}$ Excimer-Laser}

Die Messungen zur Rekonstruktion sind mit dem L1000 Excimer-Laser und dem in Abbildung (4.3) skizzierten Aufbau durchgeführt worden.

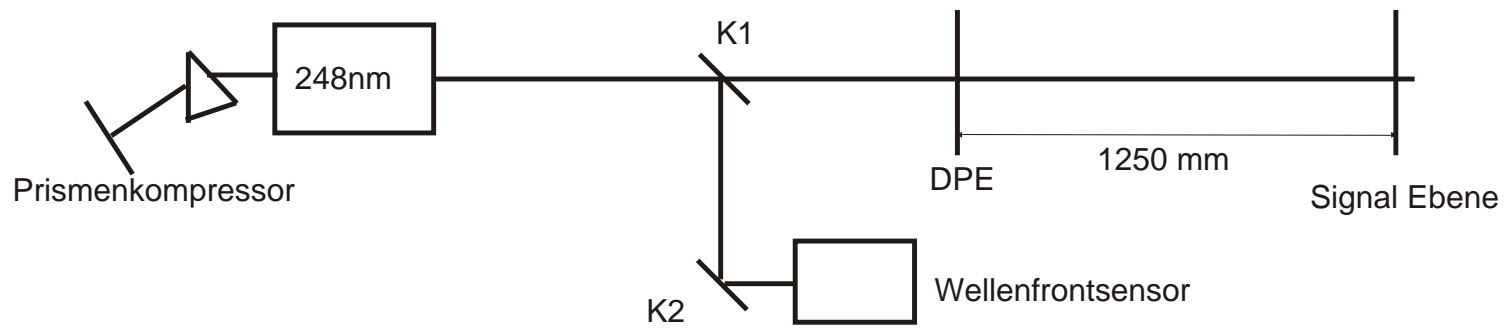

K1: Quarzkeil $6^{\circ}$

DPE: Diffraktives Phasenelement

K2: Quarzkeil 6

Abbildung 4.3: Experimenteller Aufbau DPE Nr. II/III

Die Wellenfrontcharakteristik des zur Rekonstruktion verwendeten Strahls (Abb. (4.4)a) hatte eine mittlere quadratische Abweichung von 3,7 Wellenlängen bzw. 5,1 \% zu der in das DPE eingerechneten Wellenfront (Abb. (4.2)a).

Die optische Rekonstruktion mit dem Aufbau aus Abbildung (4.3) ist in den Abbildungen (4.5),(4.6) gezeigt. 


\section{Wellenfrontcharakteristik der optischen Rekonstruktion von DPE Nr. I//III} Rohstrahl mit Prismenkompressor, L1000

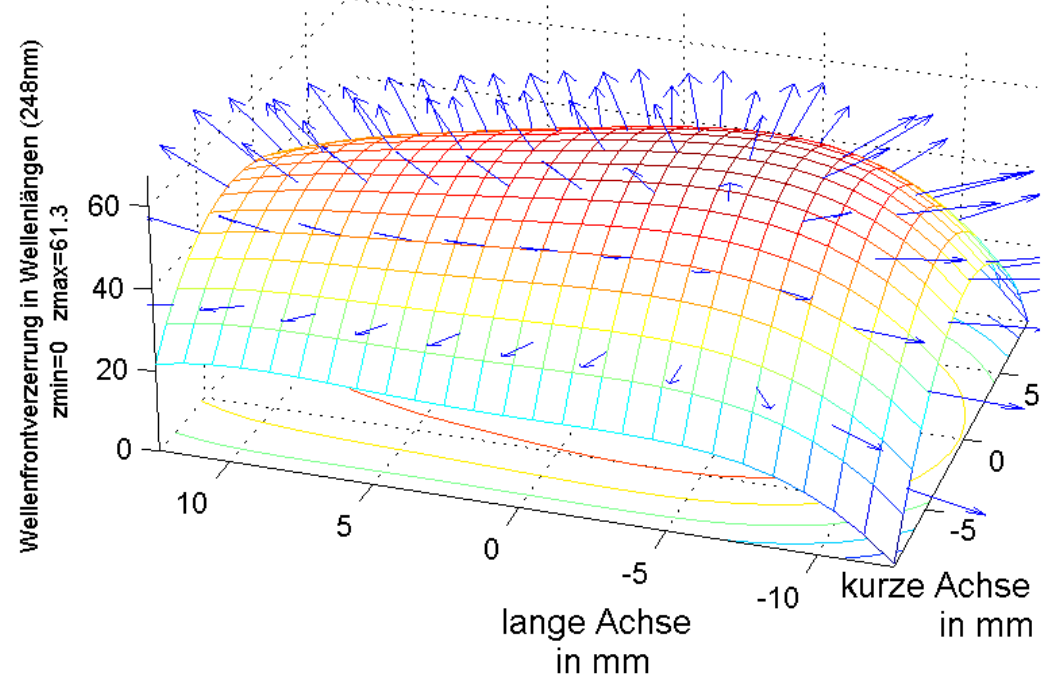

a

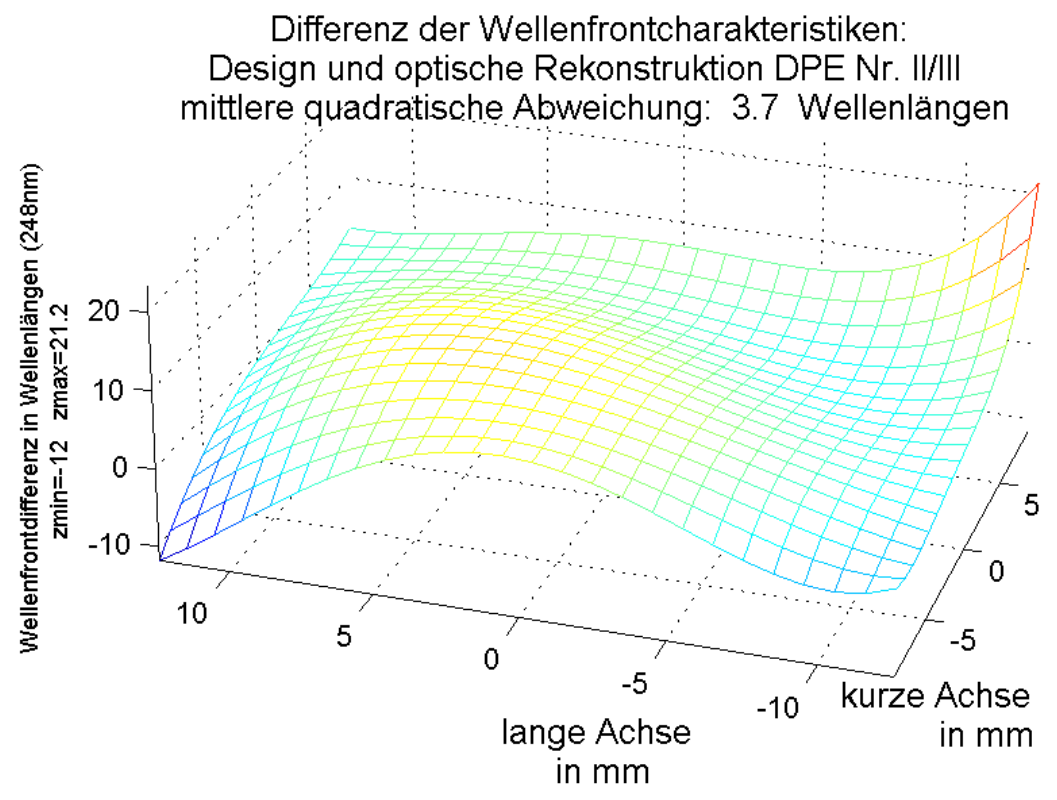

b

Abbildung 4.4: Wellenfront des zur optischen Rekonstruktion verwendeten L1000 Strahls (a). Abweichung der verwendeten Wellenfront von der in DPE Nr. II und DPE Nr. III eingerechneten Wellenfront (b). 


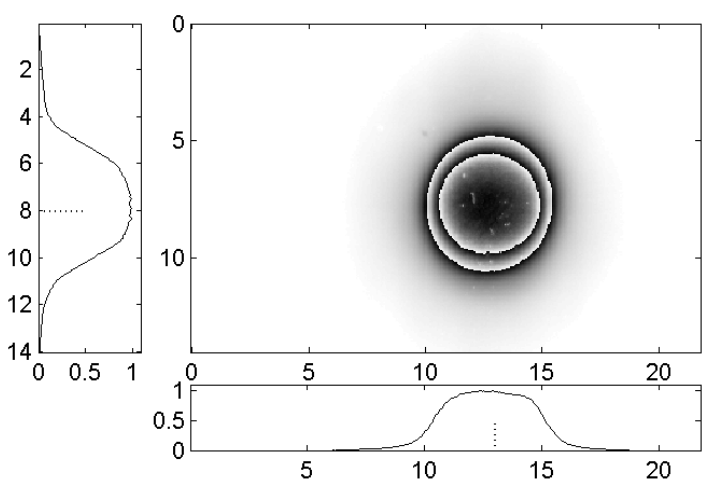

a: ganzer Strahl (77 Subperturen)

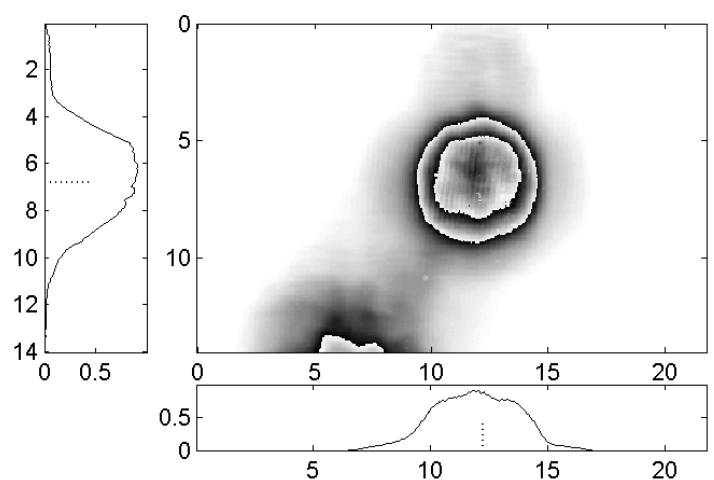

b: Subapertur $(1,-1)$

Abbildung 4.5: Optische Rekonstruktion DPE Nr. II mit KrF Excimer-Laser. Dreifache Graustufenskala, Achsen in mm.

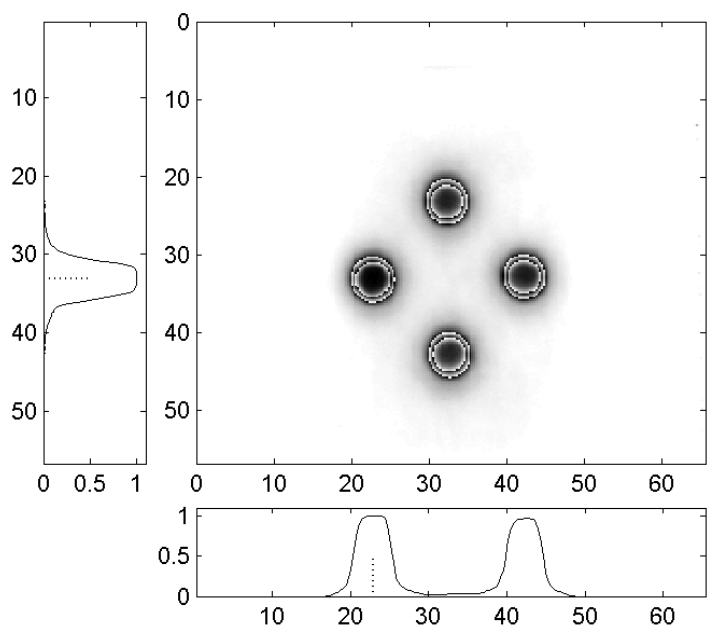

a: ganzer Strahl (77 Subaperturen)

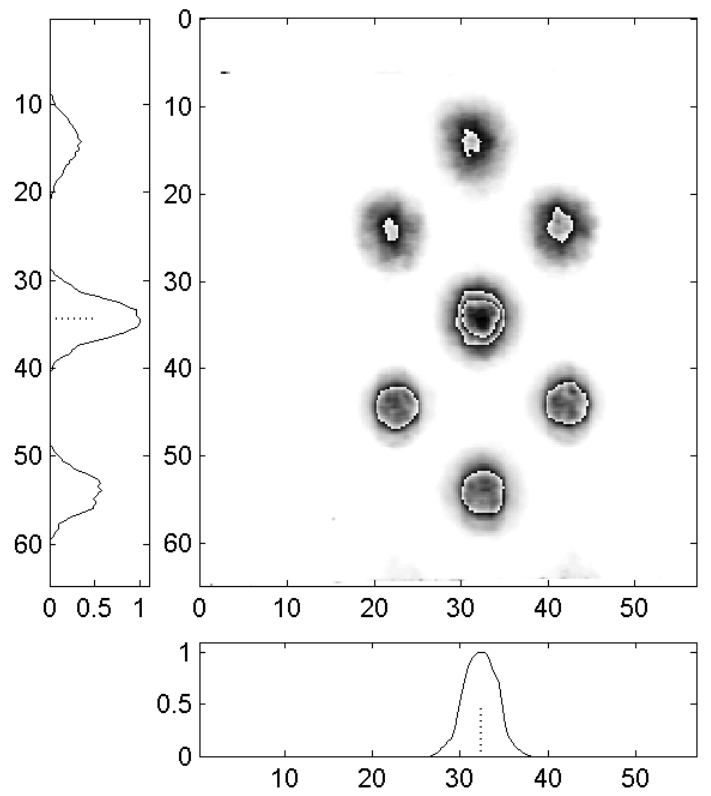

b: Subapertur $(-4,0)$

Abbildung 4.6: Optische Rekonstruktion DPE Nr. III mit KrF Excimer-Laser.

Dreifache Graustufenskala, Achsen in mm.

Die Strahlformung mit dem gesamten DPE Nr. II (Abb. (4.5)a) resultiert in einem runden Spot mit einer Halbwertsbreite (FWHM) von 5,0 mm. Die Flankensteilheit entspricht der Flankensteilheit des rekonstruierten Spots aus einer Subapertur (Abb. (4.5)b). Das 
bedeutet, die deckungsgleiche Überlagerung der Signale aus den einzelnen Subaperturen durch die Berücksichtigung der eingestrahlten Wellenfrontcharakteristik im Design des DPE ist erfolgreich realisiert worden. Dies gilt ebenfalls für die Strahlformung mit dem DPE Nr. III (Abb. (4.6)).

Die Rekonstruktion der Subapertur (-4,0) des DPE Nr. III in Abbildung (4.6)b zeigt sieben Spots. Die unteren vier Spots gehören zum gewünschten Beugungsbild. Die oberen vier Spots gehören zu einem an der optischen Achse der Subapertur gespiegelten Bild des gewünschten Beugungsbildes, d.h. der mittlere Spot liegt auf der optischen Achse der Subapertur und entsteht durch Überlagerung des oberen Spots des gewünschten Beugungsbildes und dem unteren Spot des Spiegelbildes. In der linken unteren Ecke von Abbildung (4.5)b ist ebenfalls ein solches Spiegelbild teilweise zu sehen. Diese Spiegelbilder reduzieren die Beugungseffizienz und sind auf Fehler bei der Herstellung zurückzuführen (vgl. Abschnitte 4.3 und 4.4).

\subsubsection{Effizienzmessung von DPE Nr. II/III}

Die Beugungseffizienz ist durch Pulsenergiemessungen mit einem Joulemeter bestimmt worden. In Abbildung (4.7) ist der entsprechende Aufbau skizziert. Die Messungen sind über 20 Pulse gemittelt worden.

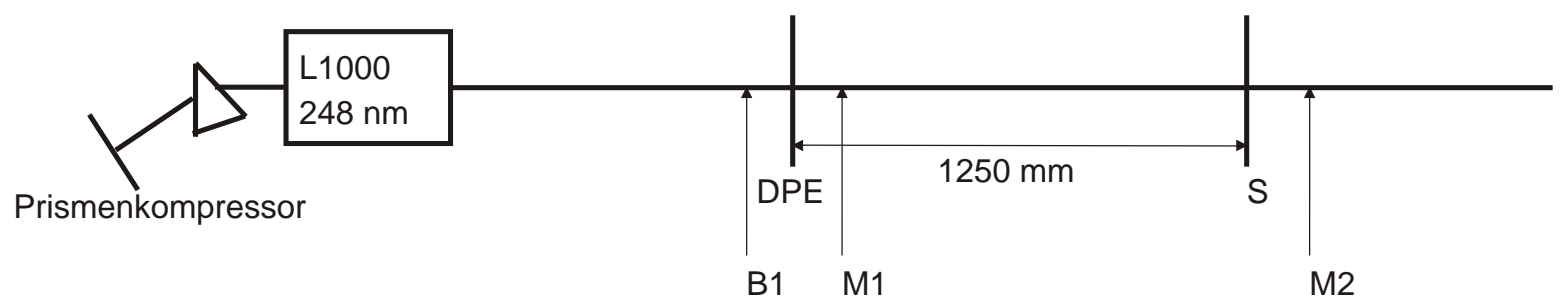

M1/ M2: Messung 1/ 2

$\mathrm{S}$ : Kreisblende $(8 \mathrm{~mm})$

B1: kreisförmige Blende (9mm), um Überstrahlung des DPE zu vermeiden.

Abbildung 4.7: Experimenteller Aufbau zur Effizienzmessung

In der folgenden Tabelle sind die Messwerte M1 und M2 zusammengestellt. Um die Energie in den vier Spots des DPE Nr. III zu bestimmen, ist die Energie eines Spots ausgemessen, und dieser Wert mit vier multipliziert worden. Dieses Vorgehen ist gerechtfertigt, da die vier Spots nur minimale Intensitätsunterschiede aufweisen (vgl. Abb. $(4.6) \mathbf{a})$. 


\subsection{Simulation der Beugungseffizienz in Abhängigkeit von Ätzfehlern für} DPE Nr. II

\begin{tabular}{l|c|c|c} 
& M1 & M2 & Beugungseffizienz $\eta$ \\
\hline DPE Nr. II & $18,9 \mathrm{~mJ}$ & $4,5 \mathrm{~mJ}$ & $4,5 \mathrm{~mJ} / 18,9 \mathrm{~mJ}=24 \%$ \\
DPE Nr. III & $27,3 \mathrm{~mJ}$ & $1,93 \mathrm{~mJ} \cdot 4$ & $7,71 \mathrm{~mJ} / 27,3 \mathrm{~mJ}=28 \%$
\end{tabular}

\subsection{Simulation der Beugungseffizienz in Abhängig- keit von Ätzfehlern für DPE Nr. II}

Die gemessenen Beugungseffizienzen für die DPE Nr. II/III, $\eta=24 \%$ bzw. $\eta=28 \%$ (Abschnitt 4.2.3), sind wesentlich geringer als für DPE Nr. I, $\eta=71 \%$ (Abschnitt 3.3.4). Die DPE Nr. I-III sind aus den gleichen Substraten mit dem gleichen Herstellungsprozess gefertigt worden, und die Werte für die theoretische Beugungseffizienz $\eta(Z)$ liegen im gleichen Bereich 58\% $\leq \eta(Z) \leq 61 \%$ (vgl. Tabellen (3.1) und (4.1)). Die gemessenen Stufentiefen (bzw. deren Standardabweichungen) von DPE Nr. II/III von $\bar{D}=127 \pm 15 \mathrm{~nm}$ bzw. $\bar{D}=120 \pm 9 \mathrm{~nm}$ weichen deutlich ab von der Stufentiefe des DPE Nr. I von $\bar{D}=$ $126 \pm 3 \mathrm{~nm}$. Insbesondere beim DPE Nr. II sind die Ätzfehler der $\pi$-Phasenstufe mit +21 nm und der $\pi / 2$-Phasenstufe mit $-5 \mathrm{~nm}$ (vgl. Tabelle (4.3)) erheblich.

Als Maß für den Ätzfehler wird das Verhältnis $\rho$ von gemessener zur Soll-Tiefe eingeführt.

$$
\begin{aligned}
& \rho_{\pi}=265 \mathrm{~nm} / 244 \mathrm{~nm}=1,09 \text { für die } \pi \text {-Phasenstufe und } \\
& \rho_{\pi / 2}=117 \mathrm{~nm} / 122 \mathrm{~nm}=0,96 \text { für die } \pi / 2 \text {-Phasenstufe. }
\end{aligned}
$$

Um die Auswirkung der Ätztiefenfehler auf die Beugungseffizienz zu modellieren, ist das folgende numerische Experiment durchgeführt worden:

Die vierstufige Phasenfunktion $\psi_{\mathrm{IFQA}} \in\left\{\frac{1}{2} \pi, \pi, \frac{3}{2} \pi, 2 \pi\right\}$ kann aus den binären Maskendaten $M_{\pi}$ und $M_{\pi / 2}$

$$
M_{\pi}=\left\{\begin{array}{l}
1 \text { für } \psi_{\mathrm{IFQA}}=1 / 2 \pi, \pi \\
0 \text { für } \psi_{\mathrm{IFQA}}=3 / 2 \pi, 2 \pi
\end{array} \quad M_{\pi / 2}=\left\{\begin{array}{l}
1 \text { für } \psi_{\mathrm{IFQA}}=1 / 2 \pi, 3 / 2 \pi \\
0 \text { für } \psi_{\mathrm{IFQA}}=\pi, 2 \pi
\end{array}\right.\right.
$$

aufgebaut werden: $\psi_{\mathrm{IFQA}}=2 \pi-\pi M_{\pi}-\pi / 2 M_{\pi / 2}$. Der Phasenwert $1 / 2 \pi$ entspricht der maximalen Ätztiefe. Die relativen Ätzfehler können nun durch $\rho_{\pi}$ und $\rho_{\pi / 2}$ berücksichtigt werden:

$$
\psi_{\mathrm{IFQA}}=2 \pi-\pi \rho_{\pi} M_{\pi}-\frac{\pi}{2} \rho_{\pi / 2} M_{\pi / 2}
$$

Nach der Propagation von $\psi_{\text {IFQA }}$ mittels der Fouriertransformation kann in der SignalEbene die Effizienz $\eta$ auf der Indexmenge des Signals bezüglich der 1. Beugungsordnung berechnet werden. Analog zu Abschnitt (3.2) wird die Effizienz $\eta(Z)$ abgeschätzt, die Beugungsverluste in höhere Ordnungen berücksichtigt: $\eta(Z)=0,81 \eta$. In Abbildung (4.8) 

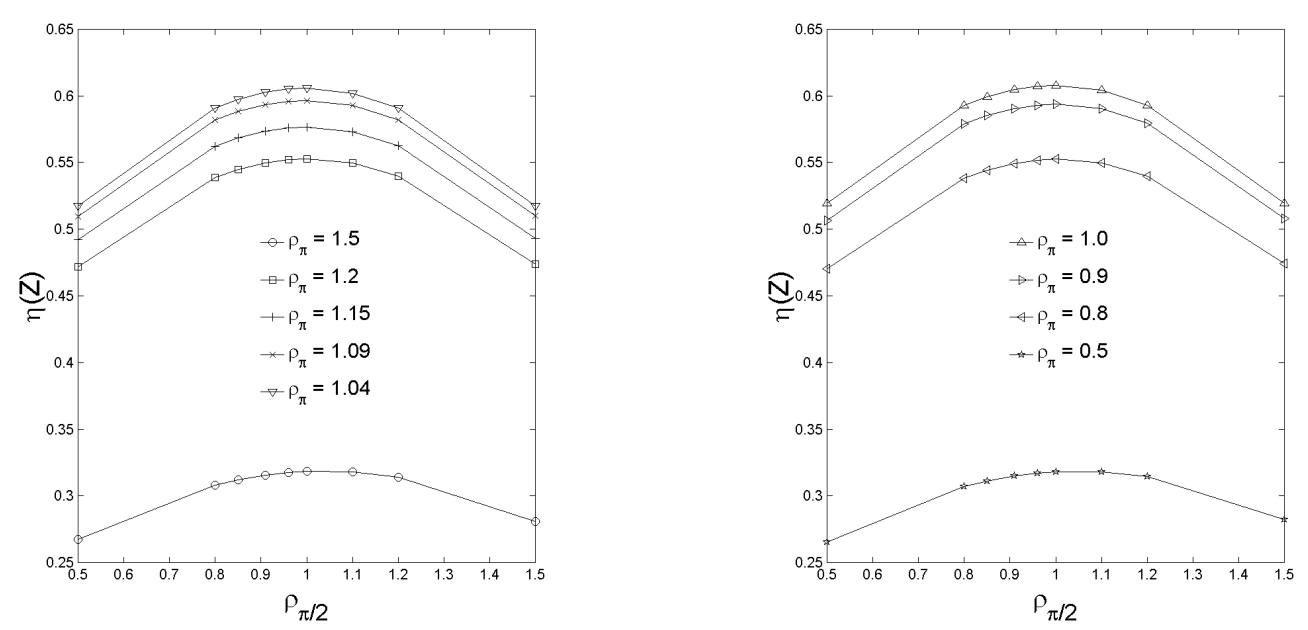

Abbildung 4.8: Simulation der Beugungseffizienz in Abhängigkeit von Ätzfehlern

sind die Ergebnisse für $\eta(Z)$ in Abhängigkeit von $\rho_{\pi}$ und $\rho_{\pi / 2}$ dargestellt. Die maximale Effizienz $\eta(Z)=61 \%$ ergibt sich für $\rho_{\pi}=\rho_{\pi / 2}=1$ in Übereinstimmung mit dem Wert aus Tabelle (4.1). Die Effizienz $\eta(Z)$ sinkt selbst für $\rho_{\pi}=1 \pm 0,2$ und $\rho_{\pi / 2}=1 \pm 0,5$ nicht unter $47 \%$. In den Bereich der gemessenen Beugungseffizienz von ca. $24 \%$ gelangt man erst bei Ätzfehlern von $50 \%$ für $\rho_{\pi}$ und $\rho_{\pi / 2}$. Dies entspricht Abweichungen der Stufenhöhe von ca. $120 \mathrm{~nm}$ bzw. $60 \mathrm{~nm}$, die weit oberhalb der Messgenauigkeit des optischen Profilometers liegen. Auch die rekonstruierte Intensitätsverteilung in Abbildung (4.9) für $\rho_{\pi}=1,09$ und $\rho_{\pi / 2}=0,96$ zeigt im Gegensatz zur gemessenen Intensitätsverteilung in Abbildung (4.5)b bzw. (4.6)b kein deutliches Spiegelbild. Diese Ergebnisse legen die Schlussfolgerung nahe, dass bei der Herstellung von DPE Nr. II/III weitere nicht erfasste Fehlerquellen, z.B. Positionierfehler der Masken, Öffnungsfehler der Masken, o.ä. vorliegen. Daraufhin sind DPE Nr. II/III eingehend mit einem Lichtmikroskop untersucht worden. Diese Untersuchungen beschreibt der folgende Abschnitt. 


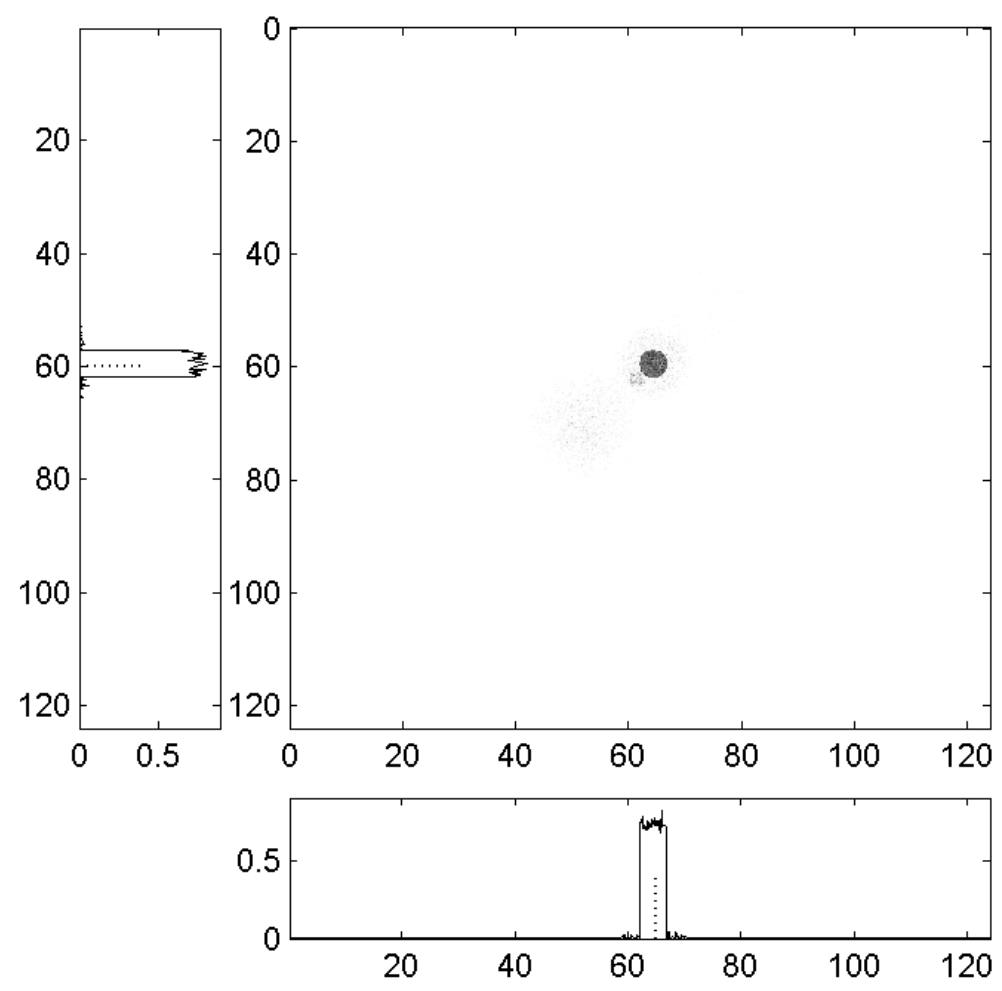

Abbildung 4.9: Simulierte Intensitätsverteilung der 1. Beugungsordnung der Subapertur $(1,-1)$ des DPE Nr. II mit $\rho_{\pi}=1,09$ und $\rho_{\pi / 2}=0,96$. Achsen in mm. Einfache Graustufenskala.

\subsection{Strukturfehler bei DPE Nr. II}

Mit einem „Zeiss Jenatech Inspection“-Lichtmikroskop wurden die DPE Nr. II/III und zum Vergleich DPE Nr. I untersucht. Aufnahmen bei einer 100-fachen Vergrößerung sind in Abbildung (4.10)a,c gezeigt. Rechts neben den Lichtmikroskopaufnahmen sind jeweils die entsprechenden Ausschnitte der berechneten Phasenfunktion $\psi_{\text {IFQA }}$ gezeigt (Abb. (4.10)b,d). Die schwarzen Pixel repräsentieren den Phasenwert $\frac{1}{2} \pi$ und entsprechen der tiefsten Ätzstufe. Die weißen Pixel repräsentieren den Phasenwert $2 \pi$ und entsprechen der obersten Stufe, d.h. einer Abdeckung in beiden Ätzschritten. Die beiden binären Masken $M_{\pi}$ und $M_{\pi / 2}$ können mit Gleichung (4.4) aus $\psi_{\text {IFQA }}$ berechnet werden. Diese Maskenberechnung aus der Phasenfunktion $\psi_{\mathrm{IFQA}}$ ist für jede Subapertur separat durchgeführt worden.

Die vorgegebene Pixelgröße von $\delta u=2,5 \mu \mathrm{m}$ ist für beide DPE sehr genau realisiert. Bei DPE Nr. I läßt sich jede Kante, die zwei Bereiche konstanter Ätztiefe voneinander trennt, eindeutig den Stufen der Phasenfunktion $\psi_{\mathrm{IFQA}}$ zuordnen. Diese Zuordnung gelingt bei DPE Nr. II nicht. Die Gebiete der Phasenfunktion $\psi_{\mathrm{IFQA}}$, die dem Ätzschritt 


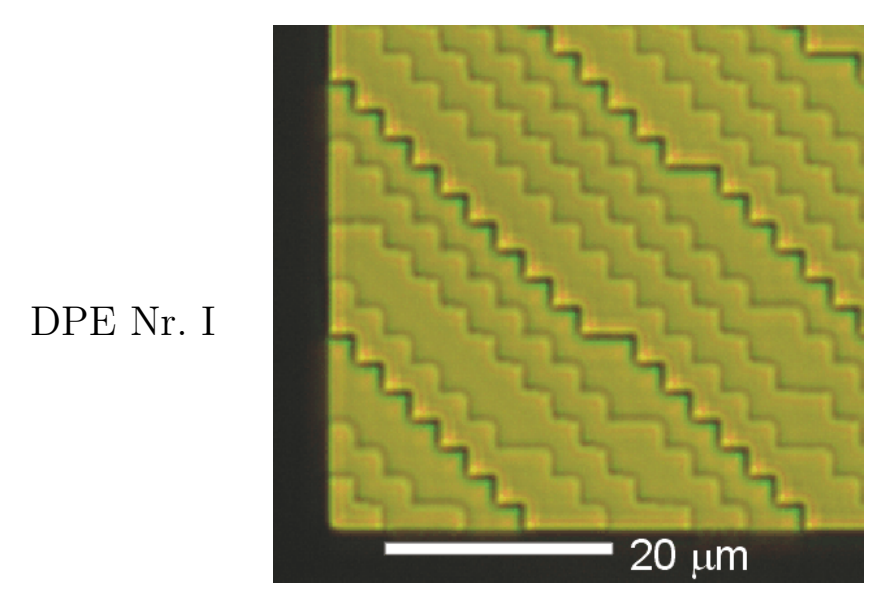

a: Lichtmikroskopaufnahme

DPE Nr. II

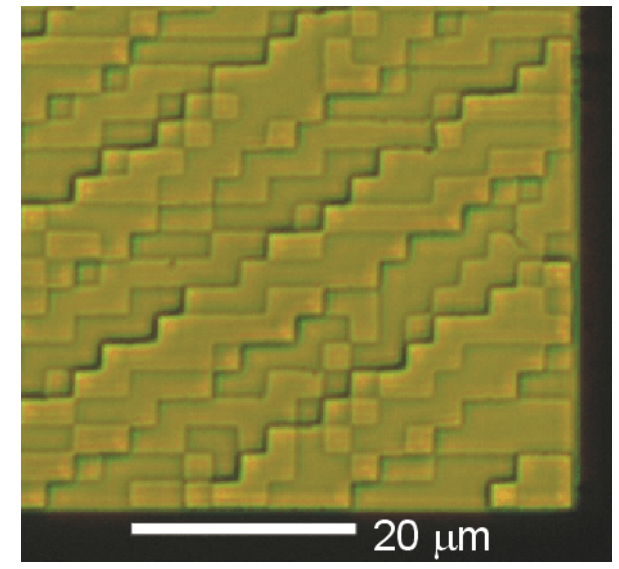

c: Lichtmikroskopaufnahme

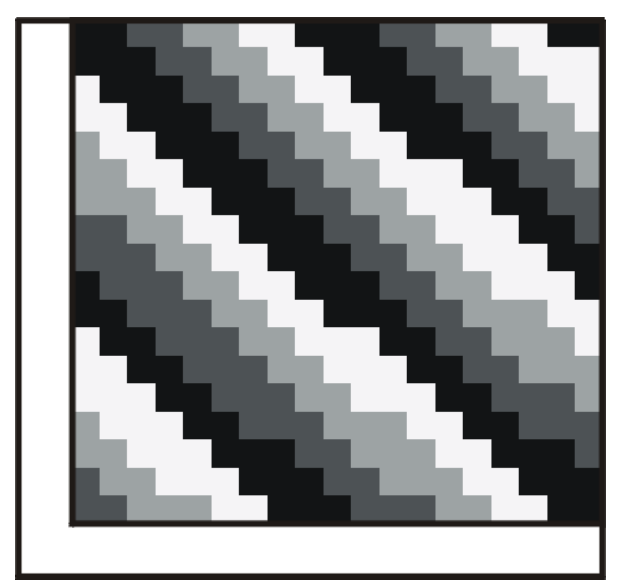

b: $\quad \psi_{\mathrm{IFQA}}$

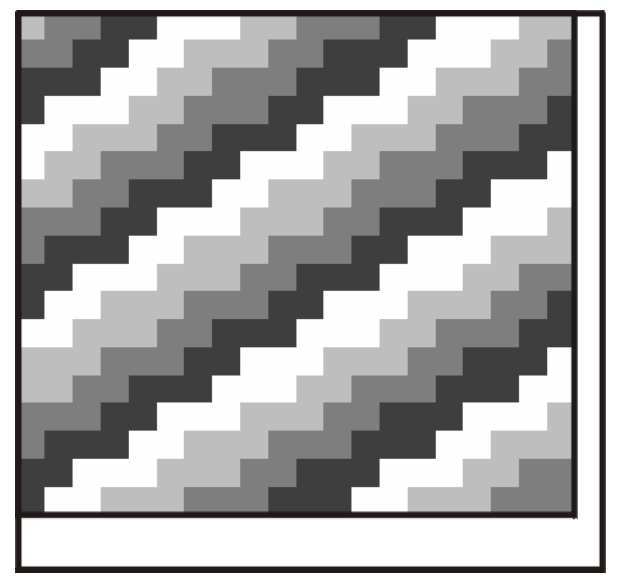

d: $\quad \psi_{\text {IFQA }}$

Abbildung 4.10: a: Lichtmikroskopaufnahme eines Ausschnitts der Subapertur (5,-5) am linken, unteren Rand des DPE Nr. I. Das Quarzsubstrat ist auBerhalb der strukturierten Fläche des DPE mit einer Chromschicht bedampft, die als schwarzer Rand sichtbar ist.

b: zu a gehörender Ausschnitt der Phasenfunktion $\psi_{\mathrm{IFQA}}$.

c: Lichtmikroskopaufnahme eines Ausschnitts der Subapertur $(5,3)$ am rechten, unteren Rand des DPE Nr. II.

d: zu c gehörender Ausschnitt der Phasenfunktion $\psi_{\mathrm{IFQA}}$.

mit der $\pi$-Maske $M_{\pi}$ entsprechen, sind durch die beiden dunklen Graustufen gegeben. Bei sorgfältiger Betrachtung gelingt es, diese Struktur in der Lichtmikroskopaufname wiederzufinden. Dieser Struktur ist ein Muster überlagert, das mit einer falschen $\pi / 2$-Maske $M_{\pi / 2}$ geätzt wurde. Die Maskendaten für jede Subapertur wurden einzeln in Dateien abgespeichert und sind bei der Herstellung offensichtlich vertauscht worden. Bei anderen 
Subaperturen des DPE Nr. II sowie bei DPE Nr. III konnte der gleiche Fehler festgestellt werden.

Dies erklärt die gemessene Reduktion der Beugungseffizienz für DPE Nr. II/III. Es mag umgekehrt erstaunlich erscheinen, dass die optische Rekonstruktion trotz dieses Fehlers noch gut funktioniert. Durch das richtige Ätzen der $\pi$-Phasenstufe entstand ein zweistufiges (binäres) DPE. Betrachtet man die fehlerhaften Pixel durch das Ätzen der $\pi / 2$ Phasenstufe vereinfachend als statistische Fehler eines binären DPE wird die "richtige“ Strahlformung plausibel. Dieses vereinfachende Modell wird gestützt durch die beobachteten Spiegelbilder nahezu gleicher Intensität (vgl. Abb. (4.6)b), da ein binäres DPE zwei Beugungsordnungen gleicher Intensität erzeugt (vgl. Tabelle(1.1)).

\subsection{Ergebnis}

Die Korrektur der divergenten Wellenfrontcharakteristik konnte erfolgreich in die DPE Nr. II/III integriert werden. Die Kantensteilheit der durch das gesamte DPE Nr. II/III geformten Strahlung entspricht der Kantensteilheit der Rekonstruktion aus einer Subapertur. Der limitierende Faktor für die Kantensteilheit ist somit durch die Kohärenzeigenschaften der Strahlung im Bereich einer Subapertur gegeben (vgl. Kapitel 5). Nach dem Kenntnisstand des Autors sind hiermit erstmalig die Daten aus experimentellen ShackHartmann Messungen zur Strahlformung mit DPE genutzt worden. Die Ursache der im Vergleich zu DPE Nr. I geringen Beugungseffizienz der DPE Nr. II/III konnte auf Herstellungsfehler zurückgeführt werden. 


\section{$5 \quad$ Korrektur der ortsaufgelösten Kohärenzeigenschaften}

Die ortsaufgelöste Korrektur der auf das DPE einfallenden Wellenfrontcharakteristik ist im vorigen Kapitel 4 behandelt worden. Es konnten damit die unterschiedlichen Hauptstrahlachsen der zueinander inkohärenten Teilbündel jeder Subapertur kompensiert werden.

In diesem Kapitel wird die Möglichkeit untersucht, die Strahlformung innerhalb jeder Subapertur zu verbessern, indem die spezifischen Kohärenzeigenschaften jedes Teilbündels berücksichtigt werden. Die Information über die räumliche Kohärenzfunktion $j$ der einzelnen Teilbündel kann aus ortsaufgelösten Fernfeldmessungen mit Hilfe des verallgemeinerten Van-Zittert-Zernike Theorems gewonnen werden (vgl. Gleichung (2.18)).

\subsection{Ortsaufgelöste Fernfeldmessungen}

Die ortsaufgelösten Fernfeldmessungen sind am Rohstrahl des L1000 Excimer-Lasers und am durch den Prismenkompressor modifizierten Strahl durchgeführt worden. In Abbildung (5.1) ist der experimentelle Aufbau gezeigt. Die quadratischen Öffnungen der Lochmas-

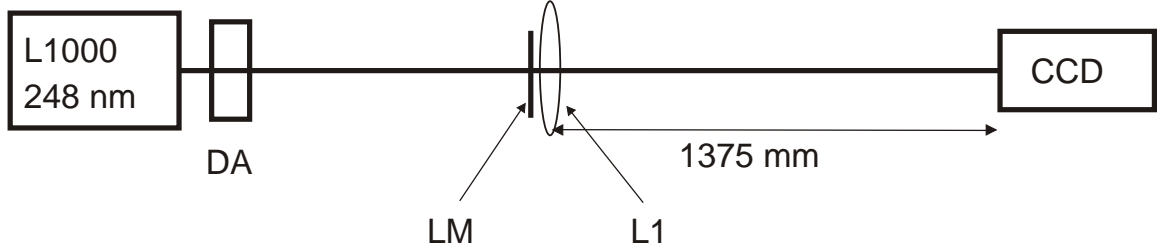

DA: Dielektrischer Abschwächer

LM: Lochmaske

L1: sphärische Linse $\mathrm{f}(248 \mathrm{~nm})=1375 \mathrm{~mm}$

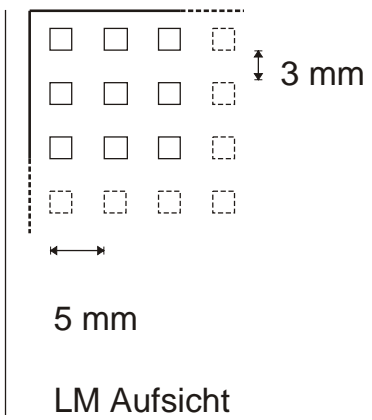

LM Aufsicht

Abbildung 5.1: Experimenteller Aufbau zur ortsaufgelösten Fernfeldmessung

ke (LM) haben eine Ausdehnung von $2.2 \mathrm{~mm}^{2}$. Ihr Abstand zueinander beträgt $5 \mathrm{~mm}$. Werden bis auf eine quadratische Öffnung alle anderen Löcher abgedeckt, ist die Messung des Fernfeldes der auf eine Subapertur des DPE einfallenden Strahlung möglich. In der Brennebene einer Linse ( $\mathrm{f}=1375 \mathrm{~mm}$ ) wird das Fernfeld mit einer UV-Strahlkamera auf 
einen CCD-Chip abgebildet. Die in den Abbildungen (5.2) und (5.3) dargestellten Fernfelder sind paraxial umgerechnet worden auf eine Brennweite $\mathrm{f}=1250 \mathrm{~mm}$ zur besseren Vergleichbarkeit mit den DPE Nr. I-IV, deren Übertragungslänge $z_{0}=1250 \mathrm{~mm}$ beträgt.

\subsubsection{Ortsaufgelöste Fernfeldmessung des L1000-Strahls mit Prismenkompressor}

Mit dem oben beschriebenen Aufbau ist aus jeder quadratischen Öffnung der Lochmaske ein Fernfeld vom L1000 Excimer-Laserstrahl mit Prismenkompressor aufgenommen worden. Die Ergebnisse sind in Abbildung (5.2) dargestellt. Die Beschriftung der Zeilen- und Spaltenkoordinaten entspricht der später für DPE Nr. IV verwendeten Nummerierung der Subaperturen.

\subsubsection{Ortsaufgelöste Fernfeldmessung des L1000-Rohstrahls}

Weitere Fernfeldmessungen sind mit dem Rohstrahl des L1000 Excimer-Lasers durchgeführt worden. Aufgrund des größeren Strahlquerschnitts und der höheren Divergenz in der langen Achse ohne Prismenkompressor konnte eine weitere Zeile der Lochmaske ausgeleuchtet werden. Die Ergebnisse sind in Abbildung (5.3) dargestellt. Es sind in der langen Achse bis zu drei voneinander unabhängige Strahlen zu beobachten, die aus einer Subapertur propagieren. 
Spalte Nr. $\rightarrow$

Zeile Nr. $\downarrow$

1
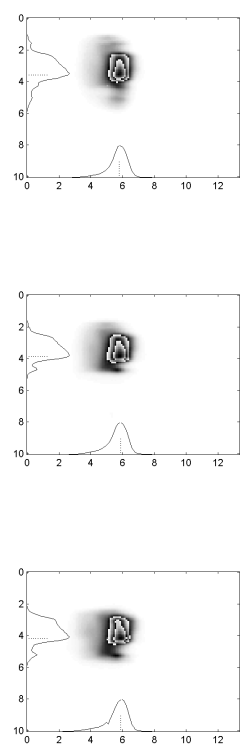

6

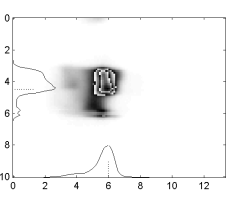

10

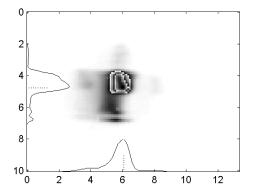

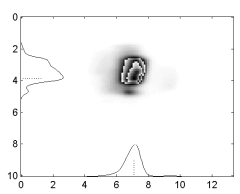

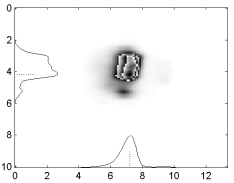

$\otimes$
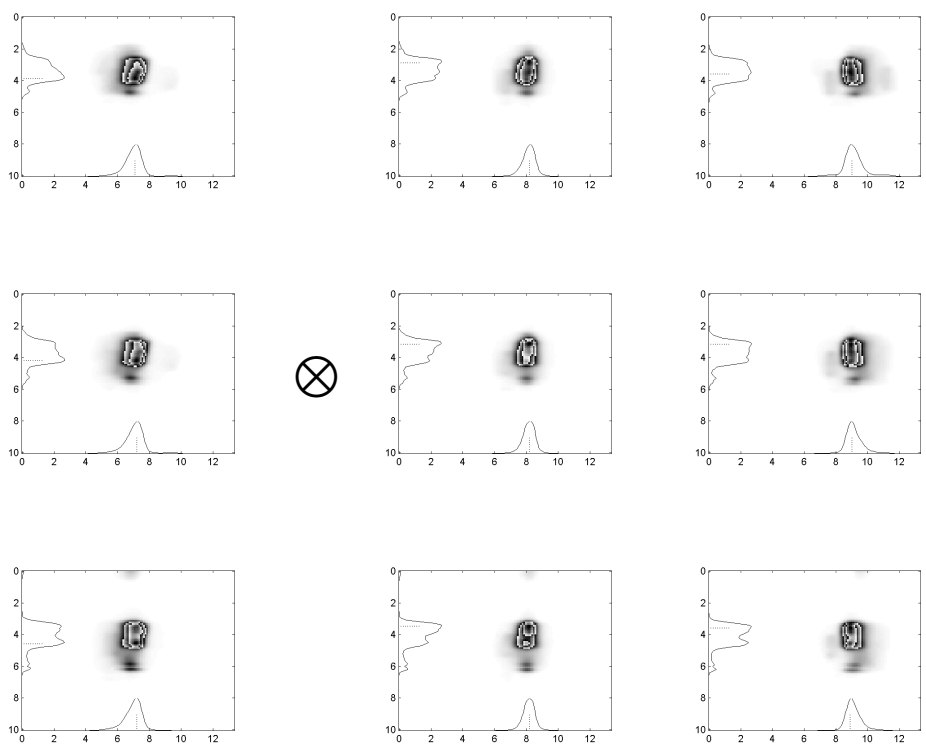

5
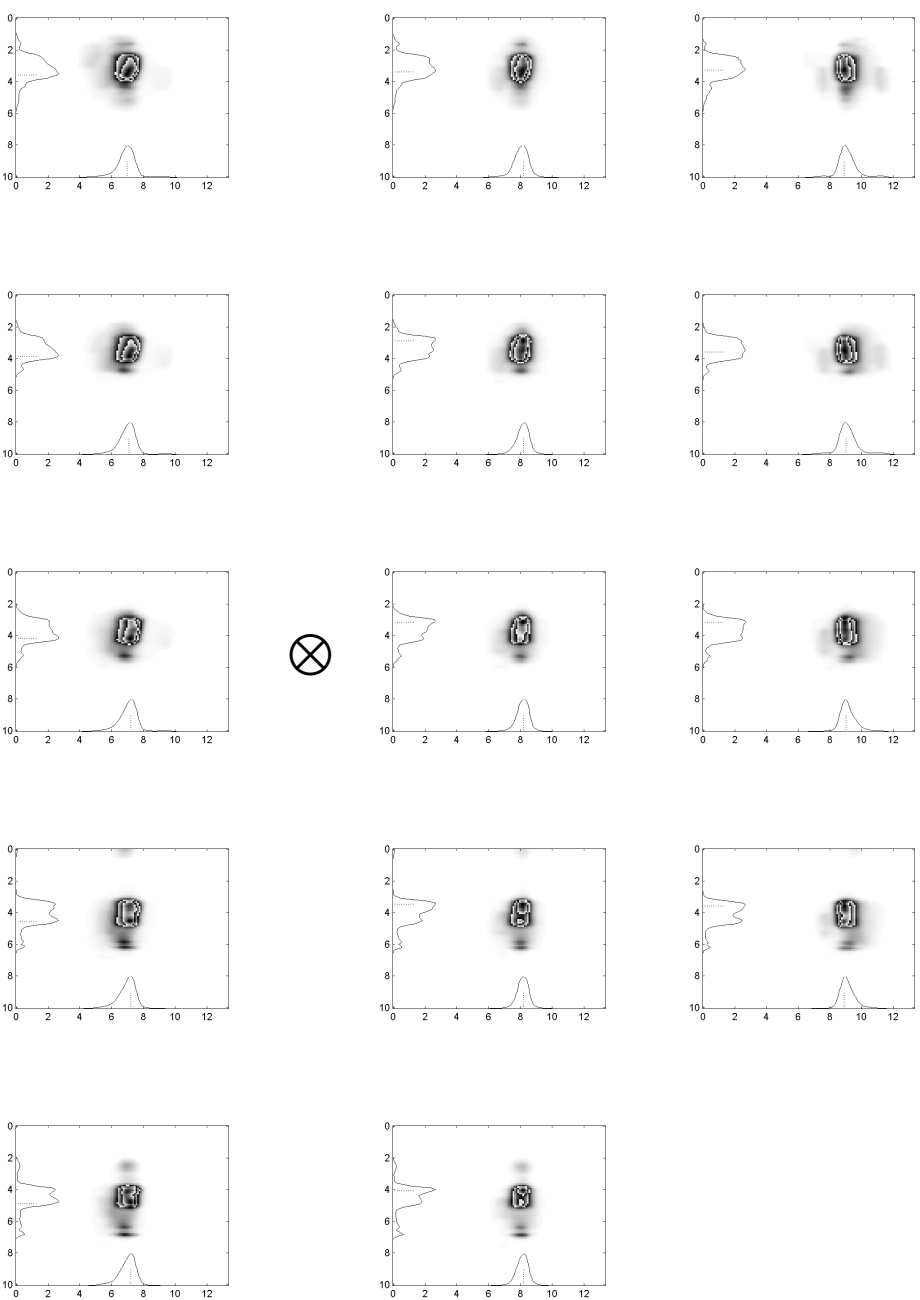

7
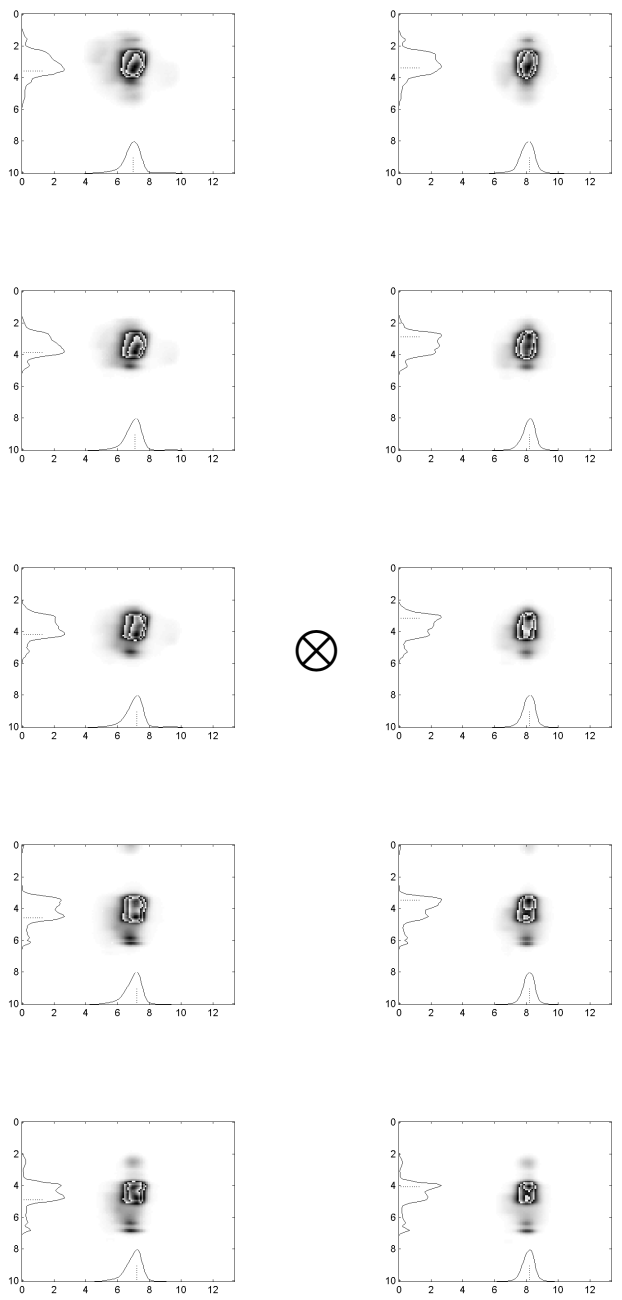

Abbildung 5.2: Ortsaufgelöste Fernfeldmessungen des L1000 Excimer-Laserstrahls mit Prismenkompressor.

Das Zeichen $\otimes$ markiert die optische Achse.

Dreifache Graustufenskala. Achsen in mm. 
Spalte Nr. $\rightarrow$

Zeile Nr. $\downarrow$

1
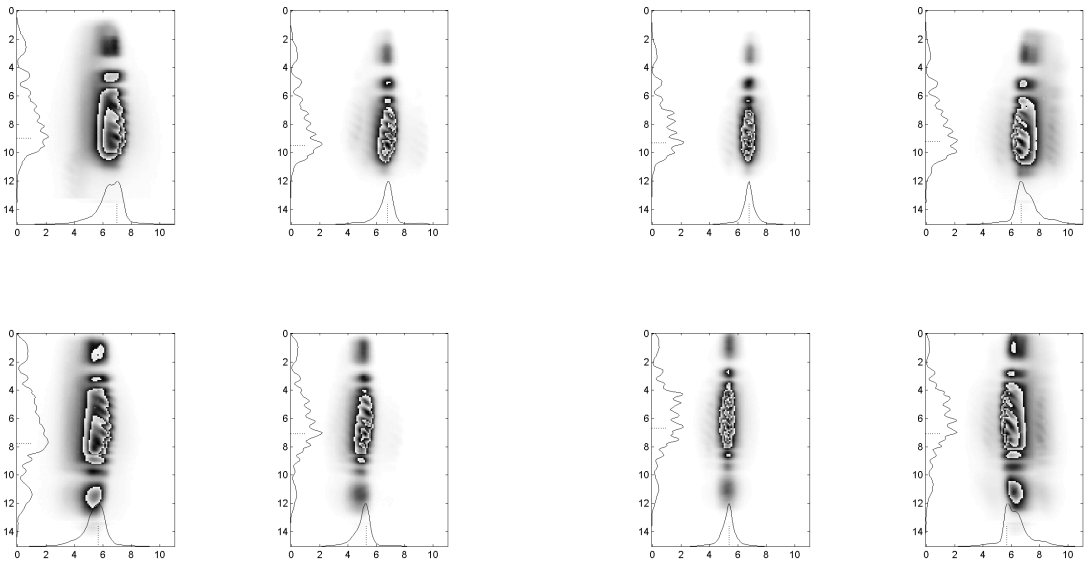

5
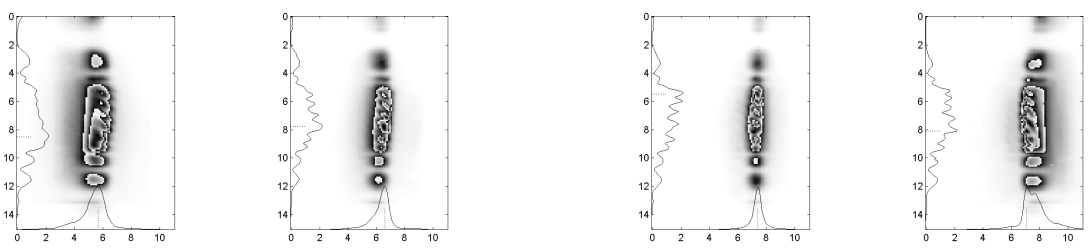

$\otimes$
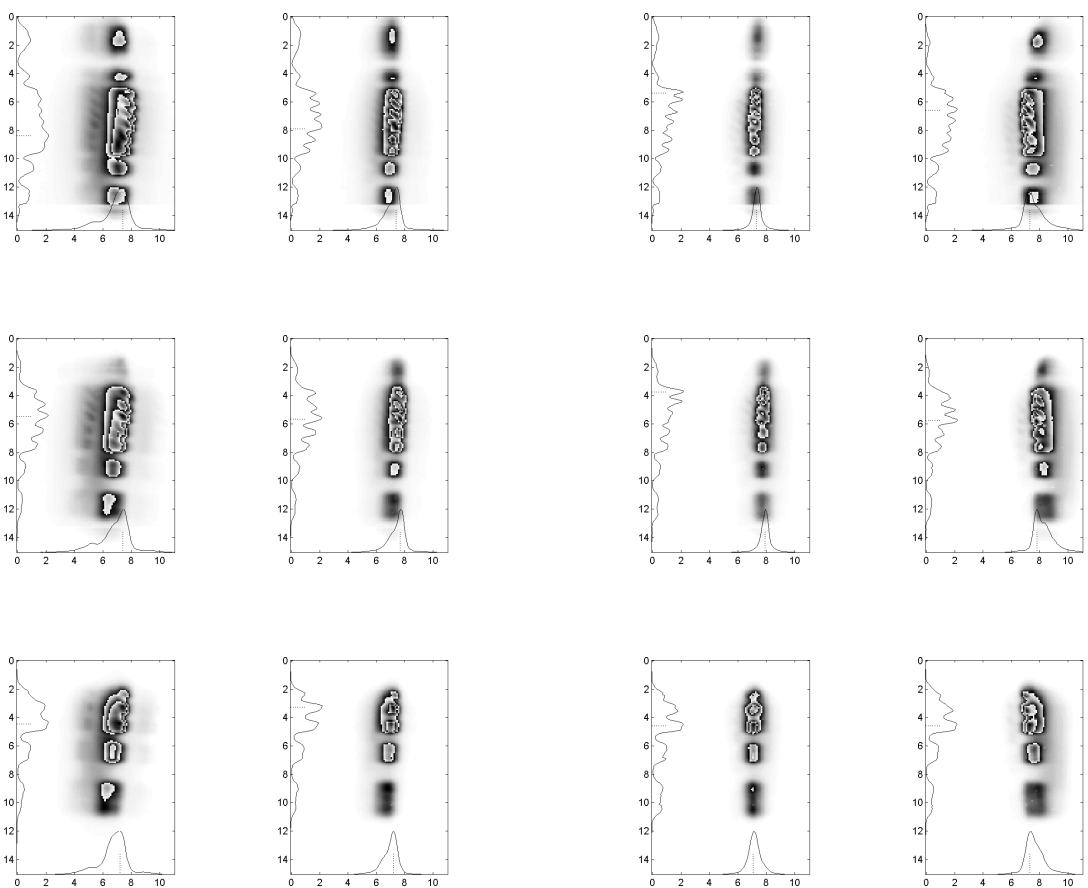

Abbildung 5.3: Ortsaufgelöste Fernfeldmessungen des L1000 - Rohstrahls

Das Zeichen $\otimes$ markiert die optische Achse.

Dreifache Graustufenskala. Achsen in mm. 


\subsection{Numerische Deconvolution}

In diesem Abschnitt wird die numerische Lösung der Gleichung (2.19)

$$
I_{\text {Faltung }}(x, y)=I_{\operatorname{coh}}(\tilde{x}, \tilde{y}) * I_{\mathrm{f}}(\tilde{x}, \tilde{y})
$$

behandelt, wobei $I_{\text {coh }}$ die (gesuchte) Intensitätsverteilung des Beugungsbildes bei kohärenter Bestrahlung darstellt und $I_{\text {Faltung }}$ die Intensitätsverteilung bei Verwendung von partiell-kohärenter Strahlung, deren Fernfeld durch $I_{\mathrm{f}}$ gegeben ist.

Diese Gleichung ist eine Fredholm Integralgleichung erster Art, stellt ein inverses Problem dar und ist im allgemeinen ,schlecht gestellt“ im Hadamardschen Sinn [35]. Ein im Hadamardschen Sinn „korrekt gestelltes“ Problem muss drei Bedingungen erfüllen: Erstens, es muss eine Lösung besitzen. Zweitens, die Lösung muss eindeutig sein und drittens stetig von den Daten abhängen. Probleme, die nicht alle drei Bedingungen erfüllen, heißen inkorrekt oder schlecht gestellt. Wie später gezeigt wird, handelt es sich bei den gegebenen Faltungskernen $I_{\mathrm{f}}$ um schlecht gestellte Probleme.

Es werden verschiedene Methoden zur näherungsweisen Lösung von (5.1) kurz erläutert, um dann den bei den gegebenen Randbedingungen erfolgreichsten Ansatz detailliert zu beschreiben. Als Testproblem wurde die Faltung eines runden Spots

$$
I_{0}(r)=\left\{\begin{array}{lll}
1 & \text { für } & r<r_{0} \\
0 & \text { für } & r>r_{0}
\end{array}\right.
$$

mit der gemessenen Fernfeldverteilung $I_{\mathrm{f}}$ aus Subapertur (4,7) des L1000 ExcimerLaserstrahls mit Prismenkompressor (vgl. Abb. (5.2)) benutzt. Dies entspricht der optischen Rekonstruktion eines diffraktiven Elements mit kohärentem Design bei partiellkohärenter Bestrahlung:

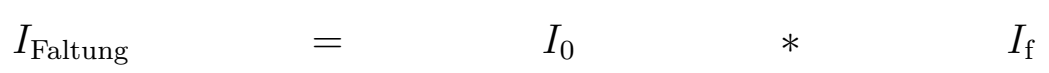
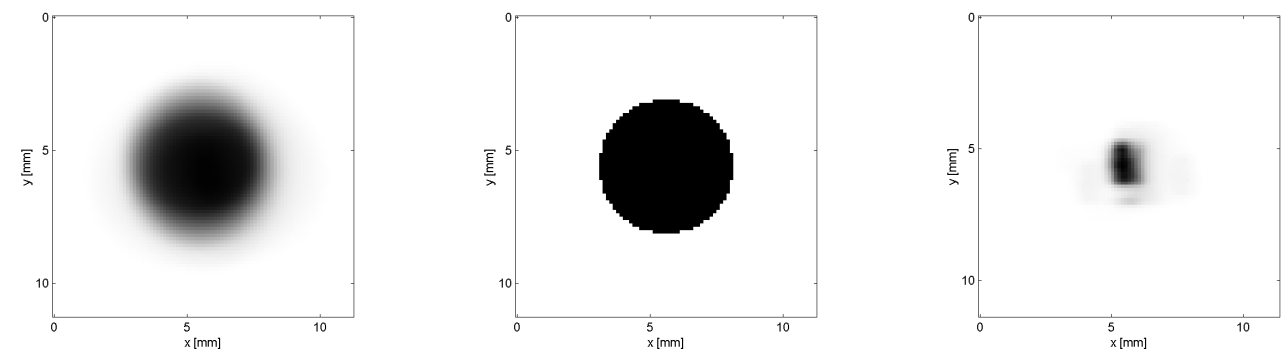

Im zweiten Schritt wurden dann die Methoden am eigentlichen Problem untersucht, eine Intensitätsverteilung $I_{\text {coh }}$ zu finden, die ein homogeneres $I_{\mathrm{pc}}$ erzeugt als $I_{\text {Faltung }}$. Das 
bedeutet $I_{\text {coh }}$ aus Gleichung (5.1) zu berechnen, wobei $I_{0}$ als $I_{\text {Faltung vorgegeben wird: }}$

$$
\begin{array}{llll}
I_{0} & I_{\mathrm{coh}} & * & I_{\mathrm{f}}
\end{array}
$$
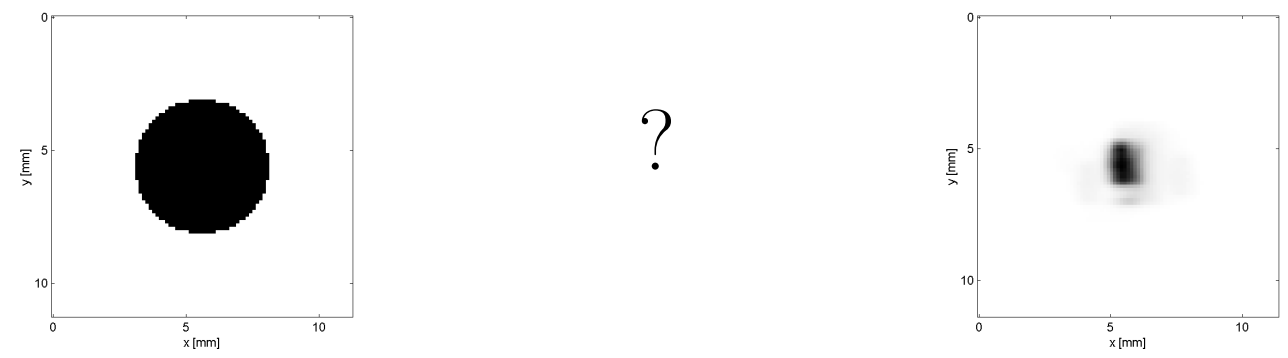

Das so berechnete $I_{\text {coh }}$ kann dann wieder in das Faltungsintegral eingesetzt werden. Es ergibt sich die Intensitätsverteilung $I_{\mathrm{pc}}$, wobei der Subscript „pc“ für partial coherence steht:

$$
I_{\mathrm{pc}}=I_{\mathrm{coh}} * I_{\mathrm{f}}
$$

Dies $I_{\mathrm{pc}}$ kann mit $I_{0}$ verglichen werden. Als Qualitätskriterium der Näherungslösungen wurde der skalierte $r m s$-Fehler $r m s_{s}\left(\underline{\underline{I_{0}}}, \underline{\underline{I_{\mathrm{pc}}}}\right)$ und die Effizienz $\eta\left(\underline{\underline{I_{0}}}, \underline{\underline{I_{\mathrm{pc}}}}\right)$ verwendet (vgl. (A.3),(A.4)).

\subsubsection{Angewendete Lösungsverfahren}

Die Beschreibung der Methoden ist unabhängig davon, ob sie auf das Testproblem oder das eigentliche Problem angewendet werden. Aus diesem Grund wird in diesem Unterkapitel $I$ als Platzhalter für $I_{\text {Faltung }}$ und $I_{0}$ verwendet.

\section{Gauss-Elimination und Matrix-Inverse.}

Die Gleichung (5.2) (bzw. (5.3)) kann in Matrixform gebracht werden (vgl. Abschnitt $5.2 .2)$ :

$$
\mathbf{I}=\underline{\underline{I_{\mathrm{f}}}} \mathbf{I}_{\mathrm{coh}}
$$

Dabei ist $\underline{\underline{I_{\mathrm{f}}}}$ die Toeplitzdarstellung des Faltungskerns [29] und $\mathbf{I}$ sowie $\mathbf{I}_{\text {coh }}$ sind die vektorisierten Versionen der entsprechenden Größen aus $(5.2,5.3)$. Diese Gleichung kann im Prinzip durch Gauss-Elimination oder durch Berechnung der Inversen von $\underline{\underline{I_{f}}}$ gelöst werden. Für schlecht gestellte Probleme ist diese Methode jedoch instabil und somit unbrauchbar.

\section{FFT-Deconvolution.}

Diese Methode nutzt das Faltungstheorem der Fouriertransformation:

$$
I_{\mathrm{coh}}=F T^{-1}\left[\frac{F T[I]}{F T\left[I_{\mathrm{f}}\right]}\right]
$$


Da die Fouriertransformierte der Fernfeldverteilung $I_{\mathrm{f}}$ fast ausschließlich kleine Raumfrequenzen beinhaltet, werden durch die Division große Raumfrequenzen durch kleine Fehler und die finite Rechengenauigkeit übermäßig verstärkt. Die Lösung $I_{\text {coh }}$ verrauscht und die Methode wird instabil. Die meisten Ansätze zur Regularisierung dieser Methode beziehen sich auf die Modellierung und Eliminierung des Rauschens (z.B. durch Wiener-Filter) des Zählers $I$, der im allgemeinen die Messdaten enthält. Die fehlerbehaftete Messgröße ist in diesem Fall jedoch der Integrationskern $I_{\mathrm{f}}$. Andere mögliche Regularisierung durch Filter in der Fourierebene wurden nicht untersucht.

\section{Iterative Deconvolution.}

Dieser nichtlineare Ansatz [29] berechnet die $(k+1)$-te Näherung $I_{\text {coh }}^{k+1}$ aus der $k$-ten Näherung :

$$
I_{\mathrm{coh}}^{k+1}=I_{\mathrm{coh}}^{k}+r(k) \cdot\left(I-I_{\mathrm{coh}}^{k} * I_{\mathrm{f}}\right)
$$

Der Relaxationsparameter $r$ bestimmt die Stärke der Korrektur in jedem Iterationsschritt und kann zum Einführen weiterer Nebenbedingungen benutzt werden. Als Startwert $I_{\text {coh }}^{0}$ kann $I$ oder eine durch andere Methoden erhaltene Lösung verwendet werden. Der Algorithmus bricht ab bei Unterschreiten des Fehlerkriteriums oder Überschreiten der maximalen Iterationsanzahl.

Numerische Experimente zeigten große Werte für $r m s_{s}$ und kleine Effizienzen $\eta$, weshalb auch diese Methode nicht weiter verfolgt wurde.

\section{Deconvolution durch Singulärwertzerlegung.}

Diese Methode erwies sich am robustesten und lieferte die besten Ergebnisse. Ihr werden deswegen die folgenden Abschnitte gewidmet.

\subsubsection{Singulärwertzerlegung und Matrixdarstellung der Convo- lution}

\section{Singulärwertzerlegung (SVD).}

Die Singulärwertzerlegung SVD (von englisch: singular value decompositon) zerlegt jede reelle Matrix $\underline{\underline{A}} \in \mathbb{R}^{n \times m}$ in zwei orthogonale Matrizen $\underline{\underline{U}}$ und $\underline{\underline{V}}$, sowie eine Diagonalmatrix $\underline{\underline{\Sigma}}$ (vgl. Lehrbücher, z.B. [21]). Die Darstellung sei hier auf den benötigten Fall $m=n$ beschränkt, dann gilt:

$$
\underline{\underline{A}}=\underline{\underline{U}} \underline{\underline{\Sigma}} \underline{\underline{V}}^{T}=\sum_{i=1}^{n} \mathbf{u}_{i} \sigma_{i} \mathbf{v}_{i}^{T}
$$




$$
\begin{array}{ll}
\text { mit: } & \underline{\underline{U}}=\left(\mathbf{u}_{1}, \ldots, \mathbf{u}_{n}\right), \quad \underline{\underline{V}}=\left(\mathbf{v}_{1}, \ldots, \mathbf{v}_{n}\right) \in \mathbb{R}^{n \times n} \\
& \underline{\underline{U}} \underline{\underline{U}}=\underline{\underline{V}} \underline{\underline{V}}=\underline{\underline{I_{n}}}, \quad \underline{\underline{\Sigma}}=\operatorname{diag}\left(\sigma_{1}, \ldots, \sigma_{n}\right) \\
& \sigma_{1} \geq \sigma_{2} \geq \ldots \geq \sigma_{n} \geq 0
\end{array}
$$

Die Singulärwerte $\sigma_{i}$ sind positiv semi-definit und in nicht-steigender Reihenfolge geordnet. Es kann gezeigt werden, dass die Anzahl der von Null verschiedenen Singulärwerte gleich dem Rang von $\underline{\underline{A}}$ ist. Die Konditionszahl cond $(\underline{\underline{A}})$ kann durch die $\sigma_{i}$ wie folgt ausgedrückt werden:

$$
\operatorname{cond}(\underline{\underline{A}})=\|\underline{\underline{A}}\|_{2}\left\|\underline{\underline{A}}^{-1}\right\|_{2}=\frac{\sigma_{1}}{\sigma_{n}}
$$

wobei $\|\underline{\underline{A}}\|_{2}$ die $p$-Norm von $\underline{\underline{A}}$ für $p=2$ bezeichnet:

$$
\|\underline{\underline{A}}\|_{p}=\left(\sum_{i=1}^{n} \sum_{j=1}^{n}\left|a_{i j}\right|^{p}\right)^{1 / p} \quad \text { mit } \quad 1 \leq p<\infty .
$$

Wenn $\underline{\underline{A}}$ singulär ist, d. h. $\operatorname{rank}(\underline{\underline{A}})<n$, dann ist $\sigma_{n}=0$ und $\operatorname{cond}(\underline{\underline{A}})=\infty$. Mit Hilfe der SVD kann nun eine formale Lösung $\mathbf{x}^{\prime}$ der Gleichung

$$
\mathbf{b}=\underline{\underline{A}} \mathbf{x}
$$

angegeben werden (vgl. z.B. [24]):

$$
\mathbf{x}^{\prime}=\sum_{i=1}^{n} \frac{\left\langle\mathbf{u}_{i}^{T}, \mathbf{b}\right\rangle}{\sigma_{i}} \mathbf{v}_{i} \quad\left(=\underline{\underline{V}} \underline{\underline{\Sigma}}^{-1} \underline{\underline{U}}^{T} \mathbf{b}\right)
$$

Diese Gleichung ist nur wohldefiniert, falls alle $\sigma_{i}>0$ bzw. größer als die Rechengenauigkeit eps sind. Für Matrizen, bei denen ab einem bestimmten Index $m$ gilt:

$$
\sigma_{i}<\text { eps für } m \leq i \leq n
$$

kann (5.7) als Ausgangspunkt zur Regularisierung genutzt werden.

\section{Toeplitz Matrizen und Faltung.}

Eine $n \times n$ Toeplitz Matrix $\underline{\underline{T}}$ ist eine Matrix, deren Elemente nur von der Differenz $i-j$ der Indices abhängen:

$$
\underline{\underline{T}}=\left(\begin{array}{ccccc}
t_{0} & t_{-1} & t_{-2} & \cdots & t_{1-n} \\
t_{1} & t_{0} & t_{-1} & \cdots & t_{2-n} \\
t_{2} & t_{-1} & t_{0} & \cdots & t_{3-n} \\
\vdots & \vdots & \vdots & \ddots & \vdots \\
t_{n-1} & t_{n-2} & t_{n-3} & \cdots & t_{0}
\end{array}\right)
$$


Es gibt $2 n-1$ unabhängige Elemente, die durch die erste Zeile und die erste Spalte festgelegt sind.

Bei der digitalen Signalverarbeitung mit von Null verschiedenen Intensitäten nur nahe der optischen Achse wie in (5.2) kann die Faltung durch Toeplitz Matrizen dargestellt werden, deren Einträge nur in einem Band mit Abstand $d$ von der Diagonalen von Null verschieden sind, genauer:

Sei $\mathbf{u}=\left(u_{1}, u_{2}, \ldots, u_{n+1}\right), n \in \mathbb{Z}^{+}$ein Vektor, der von Null verschiedene Einträge nur in einem Intervall $[n / 2+1-d, n / 2+1+d]$ um $n / 2+1$ herum besitzt. Sei $\mathbf{w}=\left(w_{1}, w_{2}, \ldots, w_{n}\right)$ ein Vektor mit von Null verschiedenen Einträgen nur im Intervall $[d+1, n-(d+1)]$. Der zentrale, von Null verschiedene Teil der Faltung $\mathbf{w} * \mathbf{u}$ ergibt sich dann durch:

$$
\mathbf{v}=\underline{\underline{T}}(\mathbf{u}) \mathbf{w} \quad \in \mathbb{R}^{n}
$$

wobei: $\quad \underline{T}(\mathbf{u})=\left(\begin{array}{ccccccc}u_{n / 2+1} & u_{n / 2} & \cdots & u_{1} & 0 & \cdots & 0 \\ u_{n / 2+2} & u_{n / 2+1} & \cdots & u_{2} & u_{1} & \cdots & 0 \\ \vdots & \vdots & \ddots & \vdots & \vdots & \vdots & \vdots \\ u_{n+1} & u_{n} & \cdots & u_{n / 2+1} & u_{n / 2} & \cdots & u_{2} \\ 0 & u_{n+1} & \cdots & u_{n / 2+2} & u_{n / 2+1} & \cdots & u_{3} \\ \vdots & \vdots & \vdots & \vdots & \vdots & \ddots & \vdots \\ 0 & 0 & \cdots & u_{n} & u_{n-1} & \cdots & u_{n / 2+1}\end{array}\right) \in \mathbb{R}^{n \times n}$

Damit das Diagonalelement in $\underline{\underline{T}}(\mathbf{u})$ wohldefiniert ist, wird der Vektor $\mathbf{u}$ so definiert, dass er $(n+1)$ Elemente besitzt. Die Bedingungen an $\mathbf{u}$ und $\mathbf{w}$ lassen sich in der Praxis durch Einbetten in entsprechend große Nullvektoren erfüllen.

Die Verallgemeinerung der Toeplitz-Darstellung der Faltung auf zweidimensionale Funktionen, bzw. Matrizen ist einfach. Die Spalten der Matrizen müssen nur übereinander gestapelt werden. Dabei ist die folgende Notation hilfreich:

Sei $\underline{\underline{X}} \in \mathbb{R}^{n \times m}$ aufgebaut aus Spaltenvektoren $\underline{\underline{X}}=\left(\mathbf{x}_{1}, \ldots, \mathbf{x}_{m}\right)$, dann wird der Vektor $\operatorname{vec}(\underline{\underline{X}})$ der Länge $n \cdot m$ definiert durch:

$$
\operatorname{vec}(\underline{\underline{X}})=\left(\begin{array}{c}
\mathbf{x}_{1} \\
\vdots \\
\mathbf{x}_{m}
\end{array}\right)
$$

Die Toeplitz-Darstellung von Gleichung (5.2) lautet damit:

$$
\operatorname{vec}\left(\underline{\underline{I_{\text {Faltung }}}}\right)=\underline{\underline{T}}\left(\operatorname{vec}\left(\underline{\underline{I_{\mathrm{f}}}}\right)\right) \operatorname{vec}\left(\underline{\underline{I_{\text {coh }}}}\right) .
$$

Diese Gleichung kann nun im Prinzip durch die SVD von $\underline{\underline{T}}\left(\operatorname{vec}\left(\underline{\underline{I_{\mathrm{f}}}}\right)\right)$ zur Berechnung von $I_{\text {coh }}$ benutzt werden. Bei einer gegebenen Bildgröße von beispielsweise $64 \times 64$ Pixeln für $\underline{\underline{I_{0}}}$ und $\underline{\underline{I_{\mathrm{f}}}}$ beträgt die Größe der zu $\underline{\underline{I_{\mathrm{f}}}}$ gehörenden Toeplitz-Matrix schon $64^{2} \cdot 64^{2} \approx$ 
$17 * 10^{6}$ Elemente. Die Matrix ist zwar dünn besetzt, doch Verwendung der StandardRechengenauigkeit von 8 Byte/Pixel führt auf einen Speicherbedarf von ca. 10-20 MB für $\underline{\underline{T}}$. Abgesehen vom Speicherbedarf wird auch die Berechnung der SVD für solche großen Matrizen unpraktikabel. Diese Probleme motivieren die im nächsten Abschnitt beschriebene Untersuchung separierbarer Faltungskerne.

\section{Zweidimensionale, separierbare Faltungskerne und ihre SVD.}

Der Faltungskern $I_{\mathrm{f}}(x-\tilde{x}, y-\tilde{y})$ heißt separabel, wenn er sich durch $I_{\mathrm{f}}(x-\tilde{x}, y-\tilde{y})=$ $I_{\mathrm{f}_{x}}(x-\tilde{x}) \cdot I_{\mathrm{f}_{y}}(y-\tilde{y})$ darstellen läßt. Die Gleichung $(5.2,5.3)$ entsprechenden Integrale werden dann zu:

$$
\begin{aligned}
I_{\text {Faltung }}(x, y) & =\iint I_{\mathrm{f}_{x}}(x-\tilde{x}) I_{\mathrm{f}_{y}}(y-\tilde{y}) I_{0}(\tilde{x}, \tilde{y}) \mathrm{d} \tilde{x} \mathrm{~d} \tilde{y} \\
I_{0}(x, y) & =\iint I_{\mathrm{f}_{x}}(x-\tilde{x}) I_{\mathrm{f}_{y}}(y-\tilde{y}) I_{\operatorname{coh}}(\tilde{x}, \tilde{y}) \mathrm{d} \tilde{x} \mathrm{~d} \tilde{y}
\end{aligned}
$$

In Matrixform läßt sich eine separierbare Matrix als Tensorprodukt zweier Vektoren darstellen:

$$
\underline{\underline{I_{\mathrm{f}}}}=\mathbf{I}_{\mathrm{fx}} \mathbf{I}_{\mathrm{fy}}^{T}
$$

Näherungsweise läßt sich eine separierte Version einer nicht-separierbaren $n \times m$ Matrix $\underline{\underline{I}}$ mit dem Separationsoperator $\operatorname{Sep} O p(\underline{\underline{I}})$ berechnen:

$$
\begin{aligned}
\mathbf{I}_{\mathrm{fx}}(i)= & S e p O p_{x}\left(\underline{\underline{I_{\mathrm{f}}}}\right)=\frac{1}{S} \sum_{l=1}^{m} \underline{\underline{I_{\mathrm{f}}}}(i, l) \text { für } i=1, \ldots, n \\
\mathbf{I}_{\mathrm{fy}}(i)= & S e p O p_{y}\left(\underline{\underline{I_{\mathrm{f}}}}\right)=\frac{1}{S} \sum_{k=1}^{n} \underline{\underline{I_{\mathrm{f}}}}(k, i) \quad \text { für } \quad i=1, \ldots, m \\
& \text { wobei } S=\sum_{k=1}^{n} \sum_{l=1}^{m} \underline{\underline{I_{\mathrm{f}}}}(k, l)
\end{aligned}
$$

Die Verwendung des Operators $S e p O p$ wird am Beispiel der Fernfeld-Intensitätsverteilung der Subapertur $(4,7)$ des L1000 Excimer-Lasers mit Prismenkompressor demonstriert $\left(I_{\mathrm{f}}\right.$ aus Gleichung $\left.(5.2,5.3)\right)$. Um die Stabilität gegenüber Messfehlern zu testen, ist die gemessene Verteilung $I_{\mathrm{f}}$ verrauscht worden. Dabei bezeichnet $r m s_{n}$ (noise) den normierten rms-Fehler (vgl. (A.2)) der verrauschten Version $I_{\text {fnoise }}$ bezüglich der ursprünglichen $I_{\mathrm{f}}$ :

$$
r m s_{n}(\text { noise })=r m s_{n}\left(\underline{\underline{I_{\mathrm{f}}}}, \underline{\underline{I_{\text {fnoise }}}}\right) .
$$

Der Fehler der Näherung von $\underline{\underline{I_{\mathrm{f}}}}$ durch $\mathbf{I}_{\mathrm{fx}} \mathbf{I}_{\mathrm{fy}}^{T}$ wird mit $r m s_{n}(\operatorname{SepOp})=r m s_{n}\left(\underline{\underline{I_{\mathrm{f}}}}, \mathbf{I}_{\mathrm{fx}} \mathbf{I}_{\mathrm{fy}}^{T}\right)$ bezeichnet. Der Fehler, der durch die Separation entsteht, liegt bei den Fernfeldverteilungen im Prozentbereich und kann im folgenden vernachlässigt werden. Es zeigt sich, 
$r m s_{n}($ noise $)=0$

$$
r m s_{n}(\text { noise })=0.05
$$

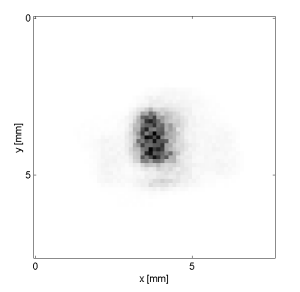

$$
r m s_{n}(\operatorname{SepOp})=0.03
$$

$r m s_{n}(\operatorname{SepOp})=0.03$

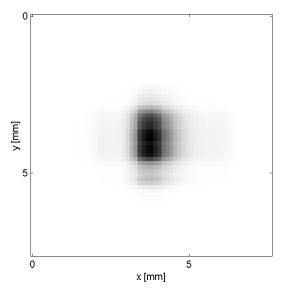

$r m s_{n}($ noise $)=0.07$

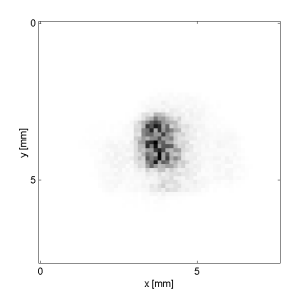

$r m s_{n}(S e p O p)=0.04$

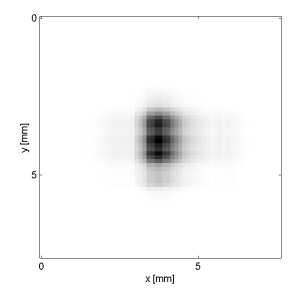

Abbildung 5.4: Der Einfluss der Separation durch SepOp und des simulierten Rauschens auf die gemessene L1000-Excimer Fernfeldverteilung mit Prismenkompressor der Subapertur (4,7). Einfache Graustufenskala.

dass die separierten Versionen der verrauschten $I_{\mathrm{f}}$ sogar geringere Fehler aufweisen als die unseparierten Versionen. Die Mittelung im SepOp-Operator glättet das Rauschen (Abb. $(5.4))$.

Es kann nun gezeigt werden [24], dass die Matrixdarstellung der Gleichung (5.13) durch die Toeplitz-Darstellungen der Vektoren $\mathbf{I}_{\mathrm{fx}}$ und $\mathbf{I}_{\mathrm{fy}}$ ausgedrückt werden kann:

$$
\underline{\underline{I_{0}}}=\underline{\underline{T}}\left(\mathbf{I}_{\mathrm{fx}}\right) \underline{\underline{I_{\mathrm{coh}}}} \underline{\underline{T}}^{T}\left(\mathbf{I}_{\mathrm{fy}}\right)
$$

Seien nun die Singulärwertzerlegungen von $\underline{\underline{T}}\left(\mathbf{I}_{\mathrm{fx}}\right)$ und $\underline{\underline{T}}\left(\mathbf{I}_{\mathrm{fy}}\right)$ gegeben durch

$$
\begin{aligned}
& \underline{\underline{T}}\left(\mathbf{I}_{\mathrm{fx}}\right)=\underline{\underline{U_{x}}} \underline{\underline{\Sigma_{x}}}{\underline{\underline{V_{x}}}}^{T}=\sum_{i=1}^{n} \mathbf{u}_{\mathbf{x} i} \sigma_{x i} \mathbf{v}_{\mathbf{x} i}^{T} \\
& \underline{\underline{T}}\left(\mathbf{I}_{\mathrm{fy}}\right)=\underline{\underline{U_{y}}} \underline{\underline{{\underline{\Sigma_{y}}}}} \underline{\underline{V_{y}^{T}}}=\sum_{i=1}^{m} \mathbf{u}_{\mathbf{y}_{i}} \sigma_{y_{i}} \mathbf{v}_{\mathbf{y}_{i}}^{T},
\end{aligned}
$$


kann damit die formale Lösung $\underline{\underline{I_{\text {coh }}^{\prime}}}$ der Gleichung (5.14) analog zu (5.7) angeschrieben werden [24]:

$$
\underline{\underline{I_{\text {coh }}^{\prime}}}=\sum_{i=1}^{n} \sum_{j=1}^{m} \frac{\left\langle\mathbf{u}_{\mathbf{x} i}^{T}, \underline{\underline{I_{0}}} \mathbf{u}_{\mathbf{y}_{j}}\right\rangle}{\sigma_{x i} \sigma_{y_{j}}} \mathbf{v}_{\mathbf{x} i} \mathbf{v}_{\mathbf{y}_{j}}^{T} .
$$

Diese Lösung $\underline{\underline{I_{\text {coh }}^{\prime}}}$ wird aus Matrizen $\mathbf{v}_{\mathbf{x} i} \mathbf{v}_{\mathbf{y}}^{T}$ vom Rang eins aufgebaut und ist nur wohldefiniert, falls alle $\sigma_{x i}, \sigma_{y_{j}}>0$. Die Anforderungen an Speicherbedarf und Rechenzeit sind verglichen mit (5.11) gering. Wenn $\underline{\underline{I_{0}}}$ und $\underline{\underline{I_{\text {coh }}}}$ als $64 \times 64$ Matrizen vorgegeben sind, ist nur die Berechnung der beiden SVD von $\underline{\underline{T}}\left(\mathbf{I}_{\mathrm{fx}}\right)$ und $\underline{\underline{T}}\left(\mathbf{I}_{\mathrm{fy}}\right)$ nötig. Diese sind ebenfalls $64 \times 64$ Matrizen. Die Gleichung (5.15) wird in den nächsten Abschnitten als Ausgangspunkt zur Regularisierung benutzt.

\subsubsection{Deconvolution durch Abbruch-Regularisierung (TSVD) mit dem Fernfeld $I_{\mathrm{f}}$ des L1000 mit Prismenkompressor.}

Falls einige Singulärwerte $\sigma_{x i}, \sigma_{y_{j}}$ in Gleichung (5.15) gleich Null oder im Bereich der Rechengenauigkeit liegen, kann der Gleichung ein Sinn gegeben werden, indem nur über die $k$ größten Singulärwert-Produkte $\sigma_{x i} \sigma_{y_{j}}$ summiert wird. Dieses Verfahren heißt „truncated“ SVD (TSVD):

$$
\underline{\underline{I^{\prime}{ }{ }_{k h k}}}=\sum_{(i, j)} \frac{\left\langle\mathbf{u}_{\mathbf{x} i}^{T}, \underline{\underline{I_{0}}} \mathbf{u}_{\mathbf{y}_{j}}\right\rangle}{\sigma_{x i} \sigma_{y_{j}}} \mathbf{v}_{\mathbf{x} i} \mathbf{v}_{\mathbf{y}_{j}}^{T}
$$

wobei die Indexpaare $(i, j)$ so gewählt sind,

dass über die $k$ größten Produkte $\sigma_{x i} \sigma_{y_{j}}$ summiert wird.

In diesem Abschnitt wird die TSVD auf die Gleichung (5.13) angewendet und deren Ergebnisse dargestellt.

Die vorgegebene Verteilung $I_{0}$ und das Fernfeld $I_{\mathrm{f}}$ bestehen aus $90 \times 90$ Pixeln, d. h. es gibt 8100 Singulärwertprodukte $\sigma_{x i} \sigma_{y_{j}}$. Ihre Verteilung in logarithmischer Darstellung ist in der folgenden Grafik zu sehen: 


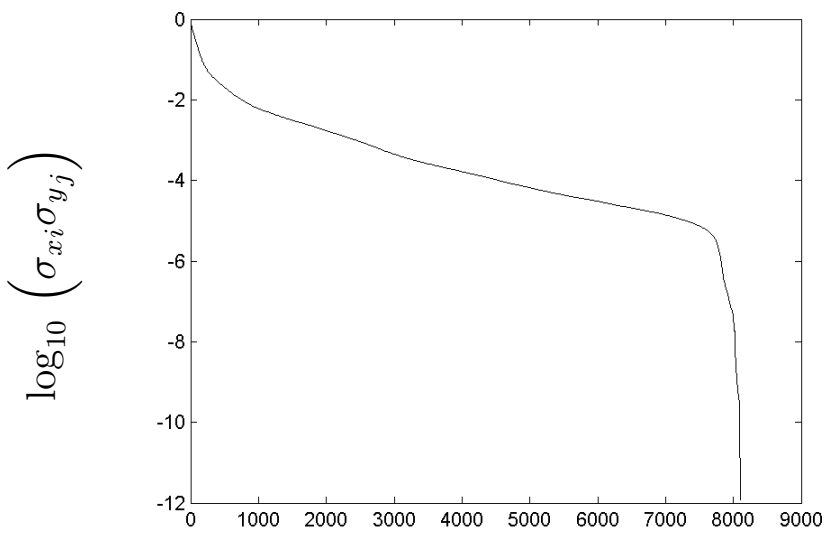

Parameter $k$
Die Sigulärwerte fallen zwar erst für $k \approx 7800$ stark $\mathrm{ab}$, höheres $k$ bedeutet aber auch ein stärkeres oszillieren der Basismatrizen $\mathbf{v}_{\mathbf{x} i} \mathbf{v}_{\mathbf{y}}^{T}{ }_{j}^{T}[24]$. Dieses Verhalten entspricht den hochfrequenten sin- und cos-Funktionen bei der Fourierentwicklung. Die besten Ergebnisse bezüglich des $r m s_{s}$-Fehlers finden sich bei $k$-Werten zwischen $150<k<350$.

Die TSVD-Regularisierung garantiert nicht, dass die Lösung $\underline{\underline{I_{\text {cohk }}^{\prime}}}$ global positiv ist. Es gibt Ansätze, Randbedingungen wie die Positivität in die Regularisierung mit einzubeziehen [24], jedoch explodiert der Rechenbedarf schon bei kleinen, eindimensionalen Problemen. Diese physikalisch erforderliche Randbedingungen für eine Intensität muss anschließend erfüllt werden, indem $\underline{\underline{I_{\text {cohk }}^{\prime}}}$ auf eine Menge mit diesen Eigenschaften

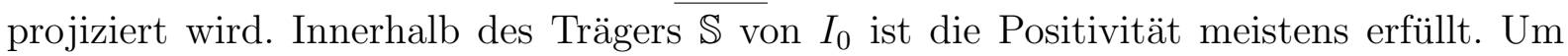
Positivität zu garantieren, wird zu $\underline{\underline{I_{\text {cohk }}^{\prime}}}$ ein Bias-Term addiert. Dann wird außerhalb von $\mathbb{S}$ die Intensität auf Null gesetzt, da die Faltung eine glättende Wirkung hat. Das Ergebnis wird geeignet normiert. Der Projektionsoperator $P=P_{1} P_{2} P_{3}$ hat also folgende dreistufige Gestalt:

$$
\begin{aligned}
& \underline{\underline{I^{\prime}}}=P_{1}(\underline{\underline{I}})=\underline{\underline{I}}-\min (\underline{\underline{I}}) \\
& \underline{\underline{I^{\prime \prime}}}=P_{2}\left(\underline{\underline{I^{\prime}}}\right)=\left\{\begin{array}{ccc}
I^{\prime}{ }_{n m} & \text { für } & (n, m) \in \mathbb{S} \\
0 & \text { für } & (n, m) \notin \mathbb{S}
\end{array}\right. \\
& \underline{\underline{I^{\prime \prime \prime}}}=P_{3}\left(\underline{\underline{I^{\prime \prime}}}\right)=\underline{\underline{I^{\prime \prime}}} \frac{\sum_{n} \sum_{m} I_{0 n m}}{\sum_{n} \sum_{m} I^{\prime \prime}{ }_{n m}}
\end{aligned}
$$

Das Ergebnis der Anwendung des Projektionsoperators $P$ auf $\underline{\underline{I_{\text {cohk } k}^{\prime}}}$ wird nun definiert als regularisierte Lösung $\underline{\underline{I_{\mathrm{coh} k}}}$ zum Parameter $k$ :

$$
\underline{\underline{I_{\mathrm{cohk}}}}:=P\left(\underline{\underline{I_{\mathrm{cohk}}^{\prime}}}\right)
$$

Diese regularisierte Lösung kann eingesetzt werden in Gleichung (5.4):

$$
I_{\mathrm{pc}_{k}}:=I_{\mathrm{coh} k} * I_{\mathrm{f}}
$$




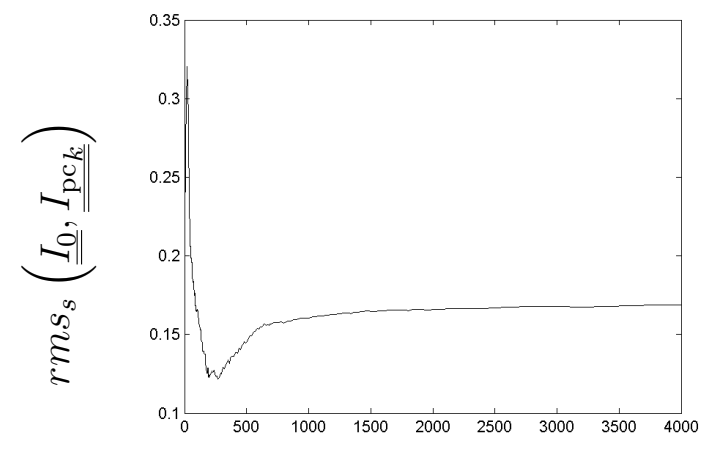

Parameter $k$

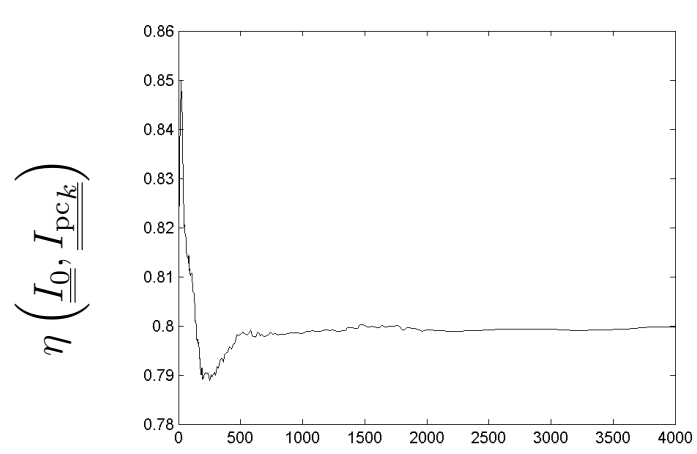

Parameter $k$

Abbildung 5.5: Skalierter rms-Fehler und Effizienz der TSVD-regularisierten Lösungen $I_{\mathrm{pc}_{k}}$.

An $I_{\mathrm{pc}_{k}}$ können nun die Qualitätskriterien $r m s_{s}$ und $\eta$ in Abhängigkeit von $k$ berechnet werden (Abb. (5.5)). Die Effizienz schwankt nur um 2-3 Prozent, der skalierte $r m s$-Fehler hingegen um ca. 10 Prozent. Der minimale $r m s_{s}$-Fehler definiert somit die beste Lösung $\mathbf{I}_{\mathrm{pc}}$ :

$$
\begin{aligned}
\underline{\underline{\mathbf{I}_{\mathrm{pc}}}:=} & \underline{\underline{I_{\mathrm{pc}_{k}}}} \\
& \text { wobei } k \text { rms }\left(\underline{\underline{I_{0}}}, \underline{\underline{I_{\mathrm{pc}_{k}}}}\right) \text { minimiert. }
\end{aligned}
$$

Verglichen werden muss der Erfolg der numerischen Deconvolution natürlich auch mit der Faltung, wenn $I_{0}$ als $I_{\text {coh }}$ vorgegeben wird. Der Notation in Gleichung (5.2) folgend, heißt das Ergebnis $I_{\text {Faltung }}=I_{0} * I_{\mathrm{f}}$. Der zugehörige $r m s$-Fehler beträgt $r m s_{s}\left(\underline{\underline{I_{0}}}, \underline{\underline{I_{\text {Faltung }}}}\right)=0.167$ und die Effizienz $\eta\left(\underline{\underline{I_{0}}}, \underline{\underline{I_{\text {Faltung }}}}\right)=0.798$. Die Lösung $\mathbf{I}_{\mathrm{pc}}=I_{\mathrm{pc} 270}$ für das obige Beispiel zeigt eine größere Homogenität $r m s_{s}=0.122$ und eine vergleichbare Effizienz $\eta=0.790$. Die Intensitätsverteilungen $I_{\mathrm{pc} 270}$ und $I_{\text {Faltung }}$ sind in Abbildung (5.6) im Vergleich gezeigt.

Es ist nicht auf den ersten Blick ersichtlich, dass der $r m s_{s}$-Fehler bei $I_{\mathrm{pc} 270}$ knapp $5 \%$

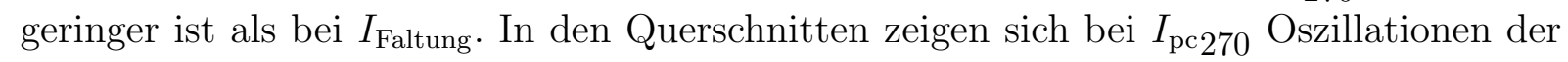
Intensität um den Plateauwert, jedoch auch steilere Flanken. Die Flanken von $I_{\text {Faltung }}$ sind auf dem Träger des $5 \mathrm{~mm}$ Spots stark abgerundet. Durch Überlagerung verschiedener Oszillationsmuster, die aus unterschiedlichen Subaperturen generiert werden, ist eine Mittelung zu erwarten. 


$$
I_{\text {Faltung }}=I_{0} * I_{\mathrm{f}}: \quad r m s_{s}=0.167, \quad \eta=0.798
$$

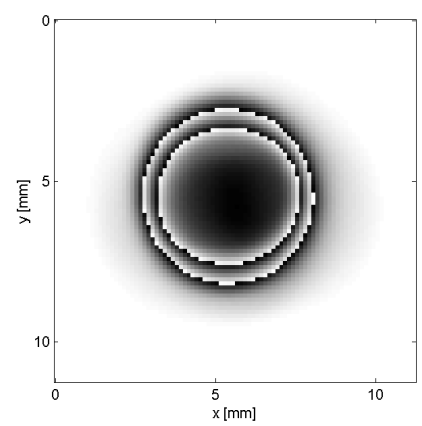

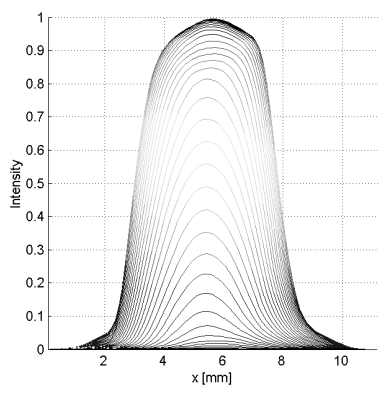

Horizontalschnitte

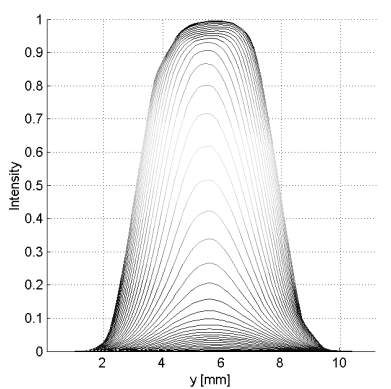

Vertikalschnitte

$$
I_{\mathrm{pc} 270}=I_{\mathrm{coh} 270} * I_{\mathrm{f}}: \quad r m s_{s}=0.122, \quad \eta=0.790
$$
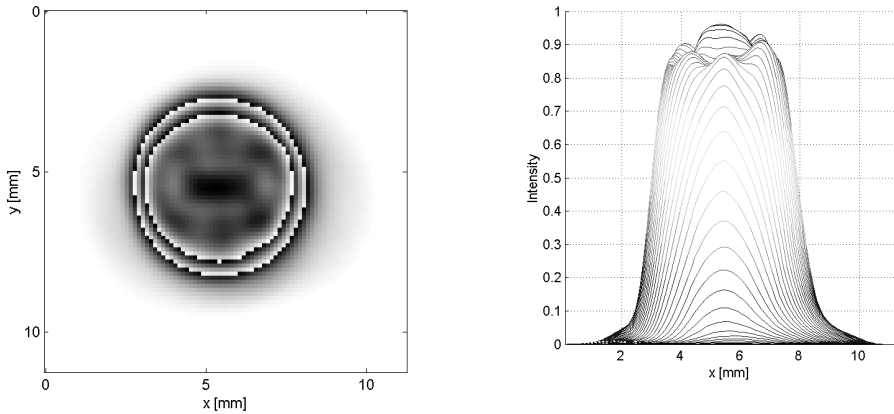

Horizontalschnitte

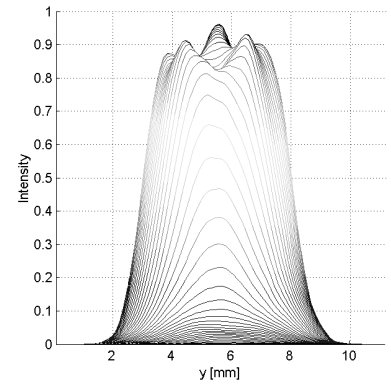

Vertikalschnitte

Abbildung 5.6: Vergleich der simulierten Rekonstruktionen aus einem „idealen“ DPE mit kohärentem Design $I_{\text {Faltung }}$ und partiell-kohärentem Design $I_{\mathrm{pc}}$ für das Fernfeld $I_{\mathrm{f}}$ des L1000 Excimer-Laserstrahls mit Prismenkompressor aus Subapertur $(4,7)$. Dreifache Graustufenskala.

Die absolute Verbesserung der Homogenität des Ergebnisses beträgt also $0.167-0.122=4,5 \%$. Die relative Verbesserung beträgt $(0.167$ $0.122) / 0.167=26,9 \%$. 
Zum Schluß dieses Abschnitts noch ein Blick auf die Intensitätsverteilung, die statt $I_{0}$ als kohärente Verteilung $I_{\text {coh }}$ vorgegeben werden muss: $I_{\text {coh270 }}$ zeigt Modulationen von \pm 0.5 um das auf 1 normierte flat-top-Profil von $I_{0}$ (Abb. (5.7)).

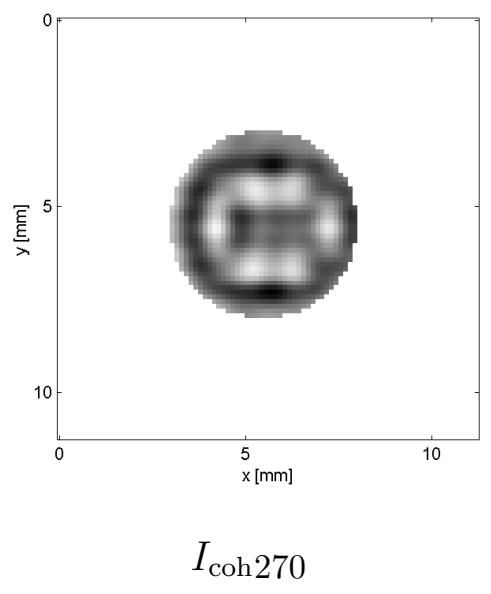

$I_{\operatorname{coh} 270}$

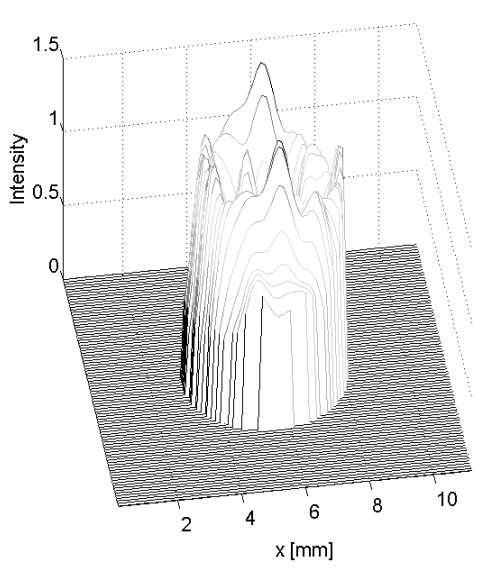

$I_{\mathrm{coh} 270}$

Abbildung 5.7: Durch den IFTA/IFQA-Algorithmus (vgl. Abschnitt (1.4)) zu realisie-

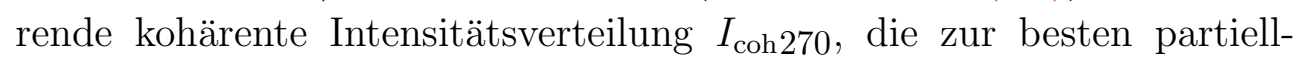
kohärenten Intensitätsverteilung $I_{\mathrm{pc} 270}$ (vgl. Abb. (5.6)) gehört.

\subsubsection{Exkurs: TSVD-Deconvolution mit dem Fernfeld des L1000 Rohstrahls}

Analog zu der TSVD-Deconvolution des Fernfeldes des L1000 mit dem Prismenkompressor in Abschnitt 5.2.3 werden in diesem Abschnitt die Möglichkeiten dieser Methode bei Anwendung auf das Fernfeld des Rohstrahls untersucht.

Als Faltungskern der Integralgleichung (5.1) wird die Fernfeldverteilung $I_{\mathrm{f}}^{\text {roh }} \operatorname{des}$ L1000 Rohstrahls aus Subapertur (3,7) verwendet (vgl. Abbildung (5.3)).

Entsprechend zur Definition in Gleichung (5.18) wird die TSVD-regularisierte Lösung $I_{\mathrm{pc} k}^{\mathrm{roh}}$ in Abhängigkeit des Abbruchparameters $k$ definiert als:

$$
I_{\mathrm{pc} k}^{\mathrm{roh}}:=I_{\mathrm{coh} k}^{\mathrm{roh}} * I_{\mathrm{f}}^{\mathrm{roh}} .
$$

Ensprechend zur Notation in Gleichung (5.2) wird die Faltung von $I_{0}$ mit $I_{\mathrm{f}}^{\text {roh }}$ benannt:

$$
I_{\text {Faltung }}^{\text {roh }}:=I_{0} * I_{\mathrm{f}}^{\text {roh }}
$$


In Abbildung (5.8) sind der skalierte rms-Fehler und die Effizienz für $I_{\mathrm{pc} k}^{\mathrm{roh}}$ in Abhängigkeit von $k$ gezeigt.

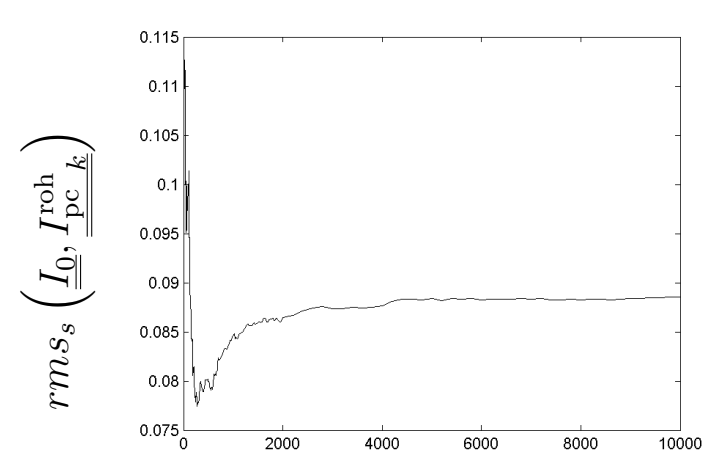

Parameter $k$

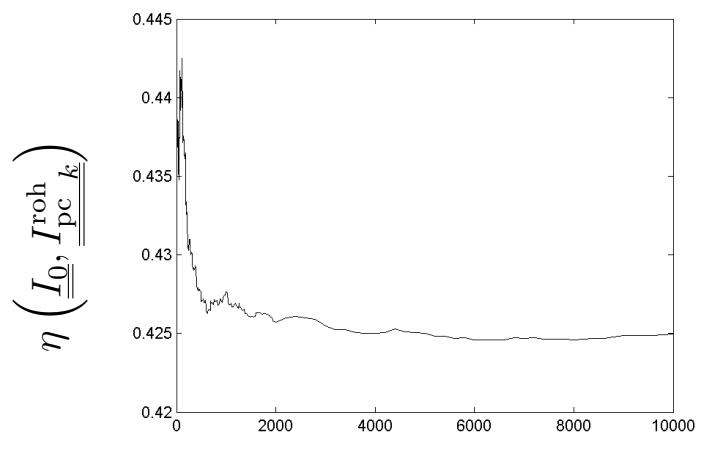

Parameter $k$

Abbildung 5.8: $r m s_{s}$-Fehler und Effizienz für TSVD-Regularisierung der Rohstrahlfernfeldverteilung aus Subapertur $(3,7)$.

Die Effizienz liegt nur bei $43 \%$, da wegen der starken Ausschmierung von $I_{\text {coh }}^{\text {roh }}$ durch die Faltung mit dem sehr langgestreckten Rohstrahlfernfeld $I_{\mathrm{f}}^{\text {roh }}$ der größere Teil der Intensität außerhalb des Trägers von $I_{0}$ liegt. Der geringste $r m s_{s}$-Fehler wird bei $k=280$ erreicht und beträgt 7,7 \%. Den Vergleich von $I_{\mathrm{pc}}^{\text {roh }} 280$ und $I_{\text {Faltung }}^{\text {roh }}$ zeigt Abbildung (5.9). Die unzureichende Strahlformung der langen Achse des Rohstrahls innerhalb einer Subapertur ist im Experiment mit DPE Nr. I beobachtet worden (vgl. Abschnitt 3.3.3 und Abb. (3.5)). In Abbildung (5.9) ist deutlich zu sehen, dass dieses Ergebnis, durch den Einsatz der TSVD-Deconvolution nicht wesentlich zu verbessern ist. Nur in der kurzen Achse (Horizontalschnitte) ist eine leichte Verbesserung festzustellen. Aus diesem und dem vorigen Abschnitt 5.2.3 läßt sich somit folgende Schlußfolgerung ziehen:

Um in einem Experiment mit einem DPE, das durch TSVD-Deconvolution ortsaufgelöste Kohärenzeigenschaften im Design berücksichtigt, einen messbaren Effekt erwarten zu können, kann der Rohstrahl des L1000 nicht als Beleuchtungsstrahl des DPE verwendet werden.

Als Beleuchtungsstrahl für das DPE kann nur der mit dem Prismenkompressor modifizierte L1000 Excimer-Laserstrahl benutzt werden. Der zu erwartende Effekt bei liegt bei $5 \%$ (absolut) bzw. $27 \%$ (relativ), (vgl. Abb. (5.6)). 


$$
I_{\text {Faltung }}^{\text {roh }}=I_{0} * I_{\mathrm{f}}^{\text {roh }}: \quad r m s_{s}=0.089, \quad \eta=0.425
$$

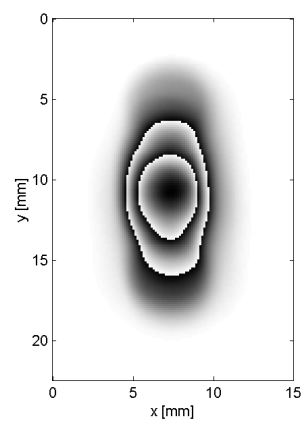

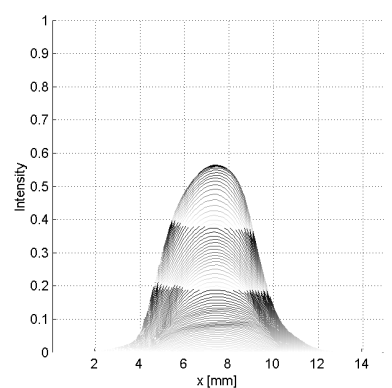

Horizontalschnitte

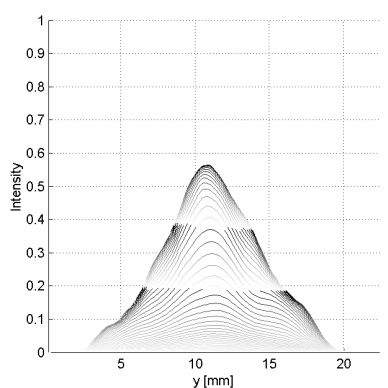

Vertikalschnitte

$$
I_{\mathrm{pc} 280}^{\mathrm{roh}}=I_{\mathrm{coh} 280}^{\mathrm{roh}} * I_{\mathrm{f}}^{\mathrm{roh}}: \quad r m s_{s}=0.077, \quad \eta=0.430
$$

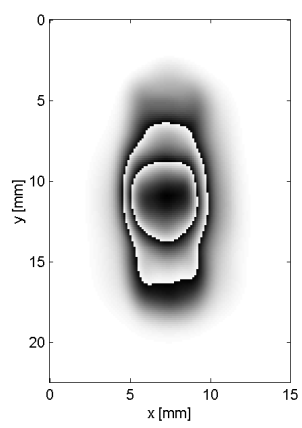

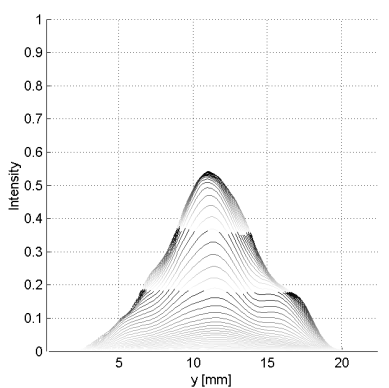

Vertikalschnitte

Abbildung 5.9: Vergleich der simulierten Rekonstruktionen aus einem „idealen“ DPE mit kohärentem Design $I_{\text {Faltung }}^{\text {roh }}$ und partiell-kohärentem Design $I_{\mathrm{pc}}^{\text {roh }}$ für das Rohstrahlfernfeld aus Subapertur (3,7) (vgl. Abb.(5.6)).

Die absolute Verbesserung der Homogenität des Ergebnisses beträgt $0.089-0.077=1.2 \%$. Die relative Verbesserung beträgt $(0.089-$ $0.077) / 0.089=13.5 \%$. 


\subsubsection{Deconvolution durch Tikhonov-Regularisierung.}

Ausgangspunkt für die Tikhonov-Regularisierung ist wie bei der TSVD-Regularisierung die Gleichung (5.15). Als Faltungskern wird wieder die Fernfeldverteilung $I_{\mathrm{f}}$ des L1000 Excimer-Laserstrahls mit Prismenkompressor verwendet. Es wird jetzt allerdings über alle Singulärwertprodukte $\sigma_{x i} \sigma_{y_{j}}$ summiert, die durch die Tikhonov-Filterfaktoren [24],[35]

$$
f_{i j}(\lambda)=\frac{\left(\sigma_{x i} \sigma_{y_{j}}\right)^{2}}{\left(\sigma_{x i} \sigma_{y_{j}}\right)^{2}+\lambda^{2}}
$$

gedämpft werden. Diese Faktoren erfüllen $0 \leq f_{i j}(\lambda) \leq 1$ und haben folgendes Verhalten, wenn $\lambda$ im Intervall zwischen dem größten und dem kleinsten Singulärwertprodukt liegt [24]:

$$
\begin{aligned}
& f_{i j}(\lambda)=1+O\left(\lambda^{2} /\left(\sigma_{x i} \sigma_{y_{j}}\right)^{2}\right) \quad \simeq 1 \quad \text { für } \quad \sigma_{x_{i}} \sigma_{y_{j}} \gg \lambda \\
& f_{i j}(\lambda)=\left(\sigma_{x i} \sigma_{y_{j}}\right)^{2} / \lambda^{2}+O\left(\left(\sigma_{x i} \sigma_{y_{j}}\right)^{4} / \lambda^{4}\right) \simeq\left(\sigma_{x i} \sigma_{y_{j}}\right)^{2} / \lambda^{2} \quad \text { für } \quad \sigma_{x i} \sigma_{y_{j}} \ll \lambda
\end{aligned}
$$

Damit wird die Tikhonovlösung zum Parameter $\lambda$ :

$$
\underline{\underline{I_{\operatorname{coh} \lambda}^{\prime}}}=\sum_{i=1}^{n} \sum_{j=1}^{m} f_{i j}(\lambda) \frac{\left\langle\mathbf{u}_{\mathbf{x}_{i}}^{T}, \underline{\underline{I_{0}}} \mathbf{u}_{\mathbf{y}_{j}}\right\rangle}{\sigma_{x i} \sigma_{y_{j}}} \mathbf{v}_{\mathbf{x} i} \mathbf{v}_{\mathbf{y}_{j}}^{T} .
$$

Analog zum obigen Abschnitt $(5.17,5.18)$ werden die folgenden Größen definiert:

$$
\begin{aligned}
\underline{\underline{I_{\mathrm{coh} \lambda}}} & :=P\left(\underline{\underline{I_{\operatorname{coh} \lambda}^{\prime}}}\right) \\
I_{\mathrm{pc} \lambda} & :=I_{\operatorname{coh} \lambda} * I_{\mathrm{f}} .
\end{aligned}
$$

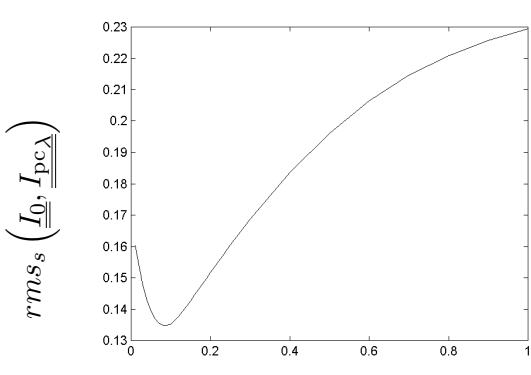

Parameter $\lambda$

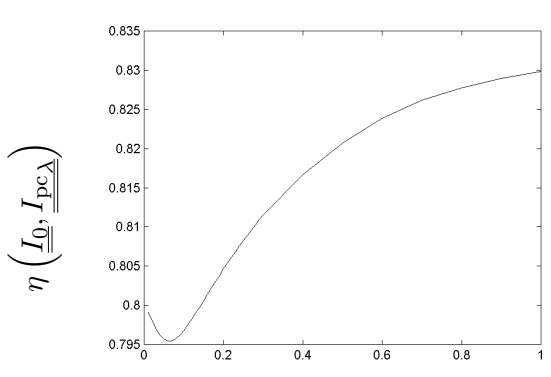

Parameter $\lambda$

Abbildung 5.10: Skalierter $r m s$-Fehler und Effizienz der Tikhonov-regularisierten Lösungen $I_{\mathrm{pc}_{\lambda}}$. Die TSVD-Lösung $I_{\text {coh270 }}$ (vgl. Abb. (5.5)) ist also der Tikhonov-Lösung $I_{\mathrm{pc}_{0.09}}$ vorzuziehen.

Der $r m s_{s}$-Fehler der Tikhonov-Lösungen hat wie die TSVD-Lösungen ein klares Minimum. Es liegt für $\lambda=0.09$ bei min $\left(r m s_{s}\left(\underline{\underline{I_{0}}}, \underline{\underline{I_{\mathrm{pc}_{\lambda}}}}\right)\right)=0.135$ jedoch rund $1.3 \%$ höher als 
das TSVD-Minimum (Abb. (5.10)). Ein weiterer Vorteil der TSVD-Regularisierung ist der wesentlich geringere Rechenaufwand. Die TSVD-Regularisierung kann in der Praxis nach dem Durchlaufen des Minimums von $r m s_{s}$, d.h. nach der Summation von ca. 1000 Singulärwertprodukten abgebrochen werden. Bei der Tikhonov-Regularisierung muß für jedes $\lambda$ über alle 8100-Singulärwertprodukte und deren Basismatrizen summiert werden (vgl. Gleichung (5.20)).

\subsection{Design und Berechnung des DPE Nr. IV}

Die Korrektur der gemessenen, ortsaufgelösten Kohärenzeigenschaften des L1000 ExcimerLaserstrahls soll nun im Design des DPE Nr. IV umgesetzt werden. Zusammengefaßt lautet das Ergebnis der Abschnitte 5.2.3 - 5.2.5, dass von den untersuchten Strahlkonfigurationen und Deconvolutionsalgorithmen die TSVD-Deconvolution und der mit dem Prismenkompressor modifizierte L1000-Laserstrahl als Beleuchtungsstrahl verwendet werden sollten. Die entsprechenden Fernfeldaufnahmen sind in Abbildung (5.2) gezeigt.

Das DPE Nr. IV soll einen $5 \mathrm{~mm}$ Spot $I_{0}$ erzeugen und besteht wie die DPE Nr. II/III aus 11.7 Subaperturen. Für die Zeilen 2-10 liegt für jede zweite Subapertur eine Fernfeldmessung vor. Die Fernfelder für die dazwischen liegenden Subaperturen werden durch lineare Interpolation aus den gemessenen berechnet. Das Design für die Subaperturen der Zeilen 1 und 11 ist aufgrund der fehlenden Fernfelder ohne Deconvolutionsalgorithmus durchgeführt worden und entspricht dem Design der Subaperturen von DPE Nr.II/III. Das Designkonzept für DPE Nr. IV und die berücksichtigten Strahleigenschaften sind im folgenden angegeben:

1. Lösen der Integralgleichung (5.3) für jede Subapertur $(i, j)$

$$
I_{0}=I_{\mathrm{coh}}^{i j} * I_{\mathrm{f}}^{i j}
$$

durch Deconvolution mit der TSVD-Regularisierung unter Verwendung der ortsaufgelösten Fernfeldaufnahmen $I_{\mathrm{f}}^{i j}$ (Abb. (5.2)) des L1000 Excimer-Laserstrahls mit Prismenkompressor.

2. Einsetzen der regularisierten Lösung $\sqrt{I_{\text {cohk }}^{i j}}$ als vorgegebene Signalverteilung $\left|f^{0}\right|$ in den IFT-Algorithmus. Das Ergebnis ist die kontinuierliche Phasenfunktion $\psi^{i j}$ (vgl. Abschnitt 1.4.1).

3. Quantisieren der kontinuierlichen Phasenfunktion $\psi^{i j}$ durch den IFQ-Algorithmus mit gleichzeitigem Einrechnen der Übertragungslänge $z_{0}$, des geometrischen Subaperturversatzes und der Wellenfrontkorrektur für den L1000-Laserstrahl mit Prismenkompressor (vgl. Abschnitte 1.4 .2 und 4.1). Das Ergebnis ist die vierstufige Phasenfunktion $\psi_{\text {IFQA }}^{i j}$. 
Die IFTA- und IFQA-Parameter sind zur Vergleichbarkeit so gewählt wie bei den DPE Nr. I-III:

\begin{tabular}{lc|lrl}
\multicolumn{2}{l|}{ IFTA-Parameter DPE Nr. IV } & IFQA-Parameter DPE Nr. IV & \\
\hline Pixelzahl N (vert.) & 996 & Pixelgröße DPE $\delta u$ & 2,5 & $\mu \mathrm{m}$ \\
Pixelzahl M (hor.) & 996 & Subaperturgröße $\Delta u$ & 2,49 & $\mathrm{~mm}$ \\
max. Iterationszahl & 110 & Übertragungslänge $z_{0}$ & 1,25 & $\mathrm{~m}$ \\
Abbruchkriterium $r m s_{s}$ & $10^{-5}$ & Pixelgröße Signal $\delta x$ & 124,5 & $\mu \mathrm{m}$ \\
& & Iterationszahl P & 45 & \\
& & Stufenzahl $Z$ & 4 &
\end{tabular}

Analog zu DPE Nr. I-III ergeben sich aus den IFTA/IFQA-Rechnungen die in Tabelle (5.1) angegebenen Werte für die Effizienz $\eta$ und den Objektfehler $r m s_{s}$.

Die Simulation der partiell-kohärenten Rekonstruktion $I_{\mathrm{pc}}^{i j}$ berechnet sich aus

$$
I_{\mathrm{pc}}^{i j}=F R T\left(\exp \left(i \psi_{\mathrm{IFQA}}^{i j}\right)\right) * I_{\mathrm{f}}^{i j} .
$$

Die $r m s_{s^{-}}$und $\eta$-Werte der partiell-kohärenten Rekonstruktion in Tabelle (5.1) sind über die 63 Subaperturen $(i, j)$ mit partiell-kohärentem Design gemittelt.

\begin{tabular}{c|c|c|c|c|} 
& \multicolumn{3}{|c|}{ DPE Nr. IV } \\
& IFTA & IFQA & Partiell-kohärente Rekonstruktion \\
\hline$r m s_{s}$ & $10^{-5}$ & $3 \times 10^{-3}$ & $r m s_{s}\left(\underline{\underline{I_{0}}}, \underline{\underline{I_{\mathrm{pc}}}}\right)=0,148$ \\
$\eta$ & $92 \%$ & $75 \%$ & $\eta(\mathrm{Z})=61 \%$ & $\eta\left(\underline{\underline{I_{0}}}, \underline{\underline{I_{\mathrm{pc}}}}\right)=0,54$
\end{tabular}

Tabelle 5.1: Über 77 Subaperturen gemittelte IFTA/IFQA-Werte für Objektfehler $r m s_{s}$ und Effizienz $\eta$.

Über 63 Subaperturen gemittelte Werte für $r m s_{s}\left(\underline{\underline{I_{0}}}, \underline{\underline{I_{\mathrm{pc}}}}\right)$ und $\eta\left(\underline{\underline{I_{0}}}, \underline{\underline{I_{\mathrm{pc}}}}\right)$.

Die Effizienz $\eta\left(\underline{\underline{I_{0}}}, \underline{\underline{I_{\mathrm{pc}}}}\right)$ aus den vierstufigen Phasenfunktionen $\psi_{\mathrm{IFQA}}^{i j}$ ist geringer als der Wert für die TSVD-regularisierte Lösung $I_{\mathrm{pc} 270}$, da bei diesen Rechnungen angenommen wurde, dass im kohärenten Beugungsbild $I_{\text {coh270 }}$ aus Abschnitt (5.2.3) $100 \%$ der Intensität enthalten sind. Der IFTA/IFQA-Algorithmus liefert für das kohärente Beugungsbild $F R T\left(\exp \left(i \psi_{\mathrm{IFQA}}^{i j}\right)\right)$ der DPE-Funkton $\psi_{\mathrm{IFQA}}^{i j}$ aber nur eine Effizienz von $\eta_{\mathrm{IFQA}}=75 \%$. Skaliert man die Effizienz $\eta\left(\underline{\underline{I_{0}}}, \underline{\underline{I_{\mathrm{pc} 270}}}\right)$ aus Abschnitt 5.2.3 (Abb. (5.6)) mit 0,75 ergibt 
sich:

$$
\eta_{\mathrm{IFQA}} \cdot \eta\left(\underline{\underline{I_{0}}}, \underline{\underline{I_{\mathrm{pc} 270}}}\right)=0,75 \cdot 0,790=0,59 \approx \eta\left(\underline{\underline{I_{0}}}, \underline{\underline{I_{\mathrm{pc}}}}\right) \quad \text { aus Tabelle } 5.1
$$

Eine analoge Argumentation erklärt auch den höheren $r m s_{s}$-Wert im Vergleich zu Abschnitt (5.2.3).

\subsection{Experiment}

Aus technischen Gründen konnten die Experimente zur optischen Rekonstruktion des DPE Nr. IV nicht mit dem identischen Laser ausgeführt werden, der für alle bisherigen Messungen an den DPE Nr. I-III und die Messungen für das Design des DPE Nr. IV verwendet wurde. Es kam stattdessen ein L1000 Excimer-Laser einer späteren Baureihe mit geringfügig veränderter Geometrie des Gasreservoirs und der Entladungselektroden zum Einsatz. Im folgenden Abschnitt werden die gemessenen Strahleigenschaften dieses Lasers dargestellt.

\subsubsection{Charakterisierung des zur Rekonstruktion von DPE Nr. IV verwendeten KrF Excimer-Laserstrahls}

Der L1000 Excimer-Laser wurde, wie im vorigen Kapitel 4 beschrieben, mit einem Prismenkompressor betrieben. Entsprechend den ortsaufgelösten Fernfeldmessungen, die für das Design des DPE Nr. IV verwendet wurden (vgl. Abb. (5.2)), ist der zur Rekonstruktion von DPE Nr. IV verwendete KrF Excimer-Laserstrahl vermessen worden. Die Ergebnisse zeigt Abbildung (5.11).

Die Fernfeld-Intensitätsverteilungen der zum Design und zur Rekonstruktion verwendeten Strahlung weisen Unterschiede auf. Die Fernfelder aus Abbildung (5.11) besitzen im Vergleich zu den entsprechenden Fernfeldern aus Abbildung (5.2) in der horizontalen Achse nur etwa die halbe Ausdehnung und in der vertikalen Achse ein annähernd gaussförmigen Querschnitt im Gegensatz zu den kastenartigen Querschnitten aus Abbildung (5.2). 
Spalte Nr. $\rightarrow$

Zeile Nr. $\downarrow$

3
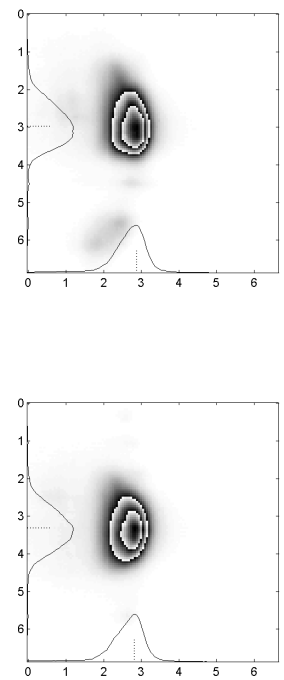

5

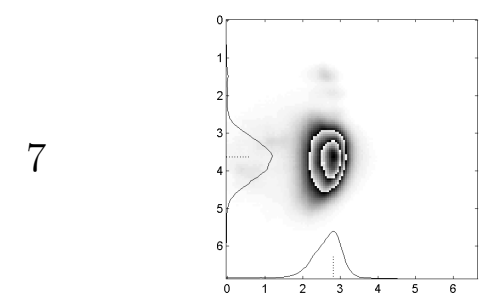

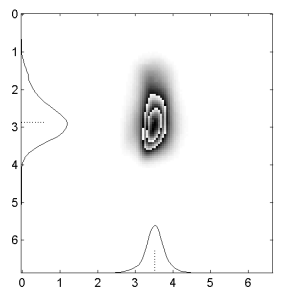

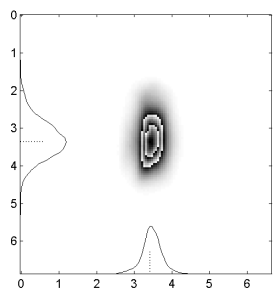

3

5
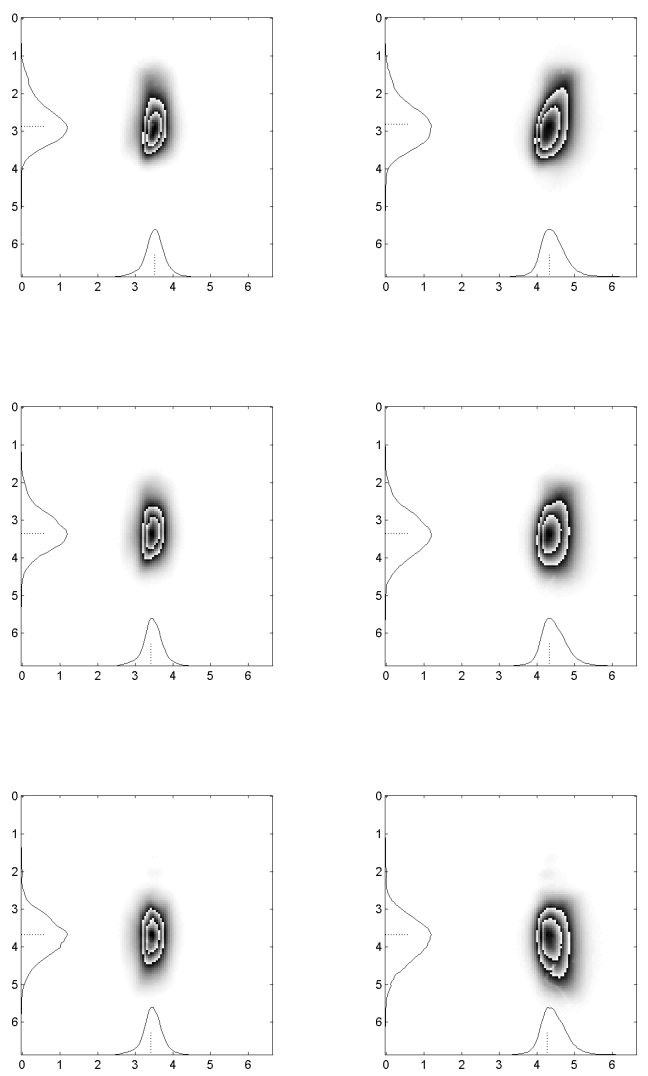

a

Abbildung 5.11:

Fernfeldmessungen des zur Rekonstruktion von DPE Nr. IV verwendeten L1000 Excimer-Laserstrahls mit Prismenkompressor.

Dreifache Graustufenskala. Achsen in mm.

a: Ortsaufgelöste Messungen einiger Subaperturen.

b: Gesamter Laserstrahl (alle Subaperturen).

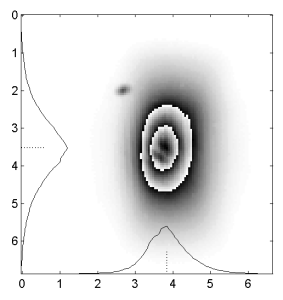


Die Wellenfrontcharakteristik des Excimer-Laserstrahls wurde mit dem gleichen Aufbau wie für DPE Nr. II/III gemessen (vgl. Abb. (4.3)) und ist in Abbildung (5.12) gezeigt. Die aus dieser Messung berechneten Divergenzen $\theta$ betragen für die kurze Achse $\theta_{x}=$ $1.6 \mathrm{mrad}$ und $\theta_{y}=1.8 \mathrm{mrad}$ für die lange Achse. Die mittlere quadratische Abweichung der Wellenfront zur in das DPE Nr. IV eingerechneten Wellenfront beträgt 3 Wellenlängen, das entspricht knapp 8\% der maximalen Auswölbung von 40 Wellenlängen.

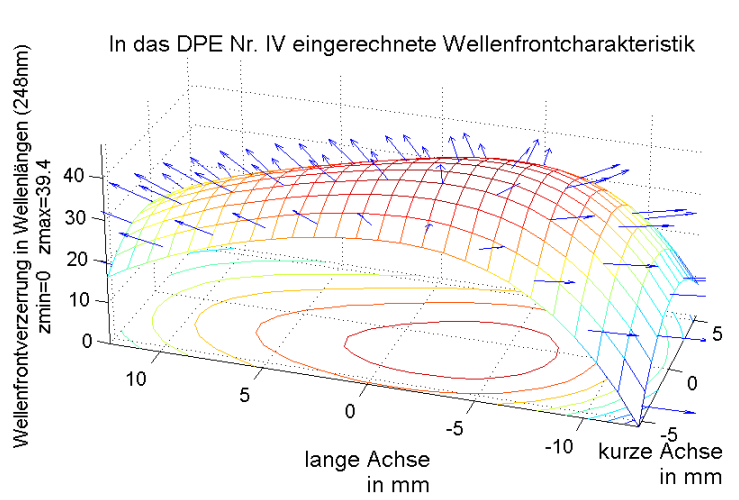

a

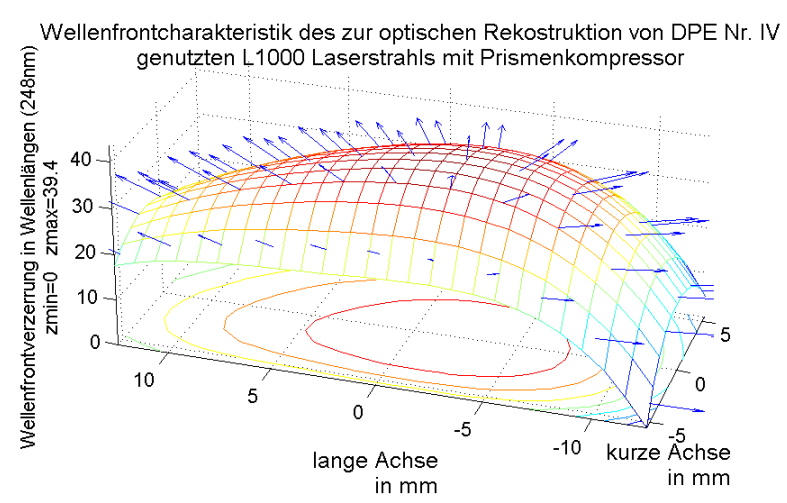

b

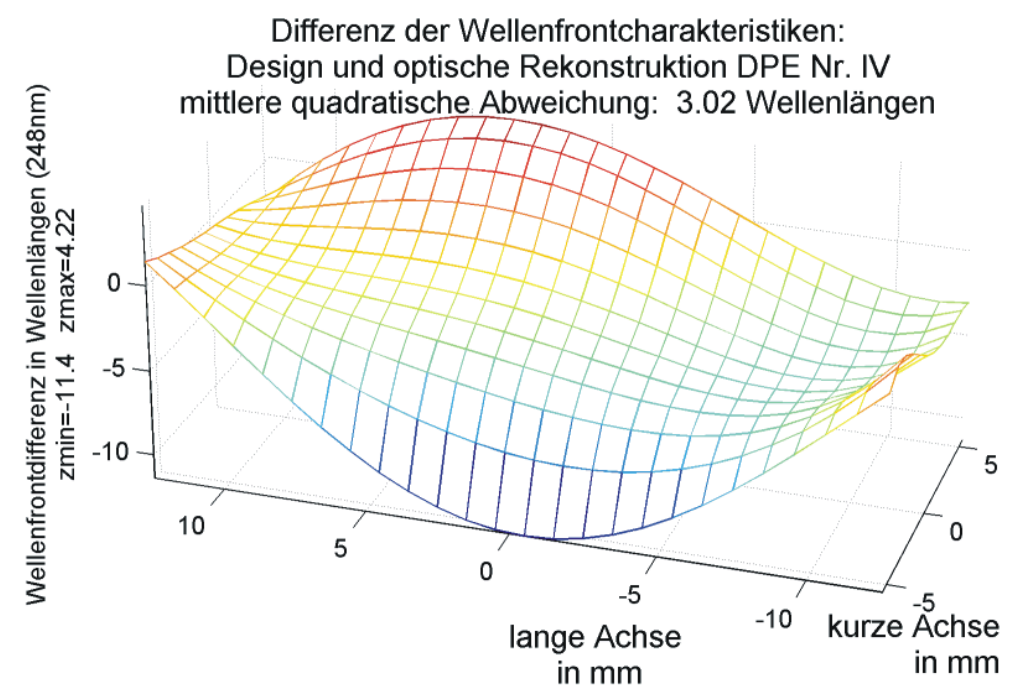

C

Abbildung 5.12: Wellenfront für DPE Nr. IV:

Design (a), Rekonstruktion (b), Differenz (c). 


\subsubsection{Optische Rekonstruktion des DPE Nr. IV mit KrF Excimer-Laser}

Die Strahlformung mit dem gesamten DPE Nr. IV (Abb. (5.13)a) resultiert in einem runden Spot mit der Halbwertsbreite (FWHM) von 5,1 mm. Um ein quantitatives Maß für die Flankensteilheit des Spots zu erhalten, ist die mittlere Flankenbreite berechnet worden. Die Flankenbreite ergibt sich aus dem Abstand der Positionen im Querschnitt-Graphen, an denen die Intensität $10 \%$ bzw. $90 \%$ der maximalen Intensität annimmt. Für den horizontalen und den vertikalen Querschnitt erhält man je zwei Werte über die gemittelt wurde. Damit folgt für den Spot in Abbildung (5.13)a eine mittlere Flankenbreite von 1,4 mm. Die mittlere Flankenbreite des aus einer Subapertur geformten Spots (vgl. Abb. (5.13)b) ist mit 1,2 mm nur geringfügig kleiner. Die Korrektur der Wellenfront ist somit näherungsweise realisiert worden.

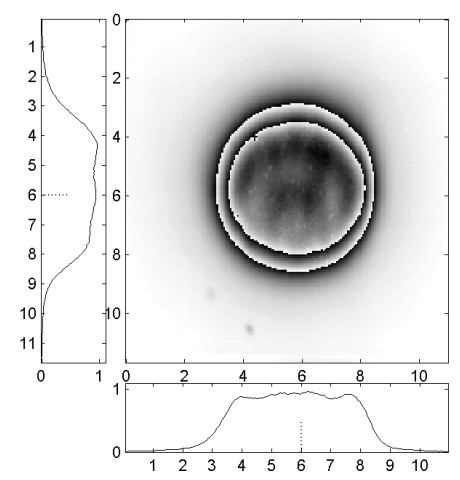

a: ganzer Strahl (77 Subperturen)

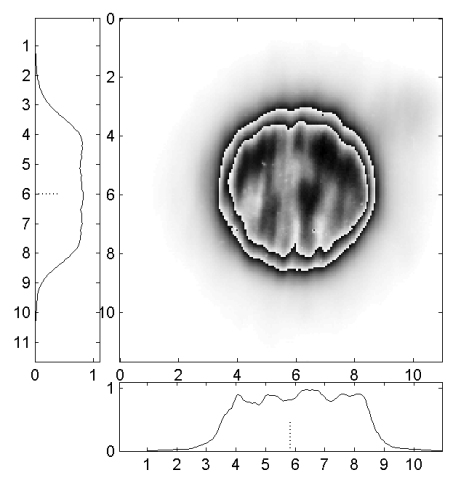

b: Subapertur $(5,5)$

Abbildung 5.13: Optische Rekonstruktion DPE Nr. IV mit KrF Excimer-Laser.

Dreifache Graustufenskala, Achsen in mm.

Um abschätzen zu können, ob die Berücksichtigung der ortsaufgelösten Fernfelder im Design des DPE Nr. IV einen signifikanten Einfluss auf die optische Rekonstruktion hat, ist mit DPE Nr. II eine Vergleichsmessung durchgeführt worden. Die in das DPE Nr. II eingerechnete Wellenfront weicht im quadratischen Mittel nur 3,6\% von der in DPE Nr. IV integrierten Wellenfront ab. Das DPE Nr. II ist jedoch unter der idealisierten Annahme vollständiger Kohärenz im Bereich einer Subapertur entworfen worden. Dies entspricht einem idealisierten, punktförmigen Fernfeld für die auf jede Subapertur treffende Strahlung. In Abbildung (5.14) ist das entsprechende Strahlformungsergebnis gezeigt. 


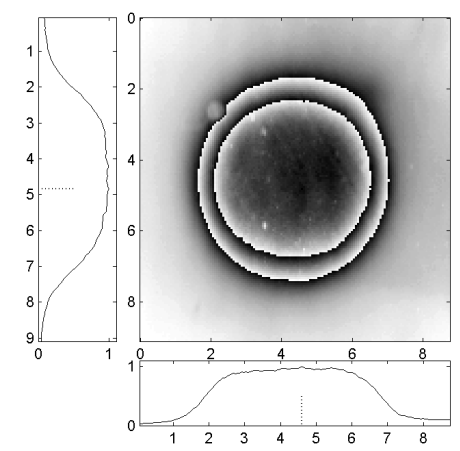

a: ganzer Strahl (77 Subperturen)

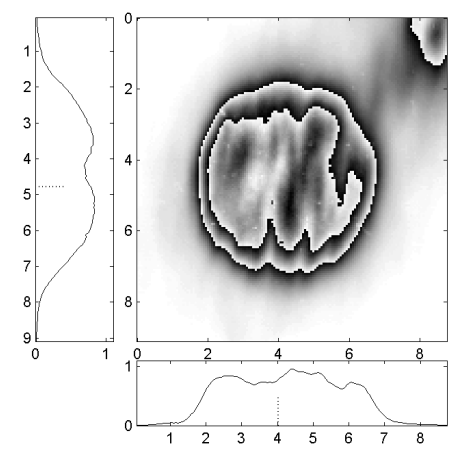

b: Subapertur $(5,5)$

Abbildung 5.14: Vergleichsmessung zum Strahlformungsexperiment mit DPE Nr. IV aus Abbildung (5.13).

Optische Rekonstruktion DPE Nr. II mit KrF Excimer-Laser.

Dreifache Graustufenskala, Achsen in mm.

Die Strahlformung mit dem gesamten DPE Nr. II (Abb. (5.14)a) resultiert in einem Spot mit der Halbwertsbreite (FWHM) von 5,0 mm. Die mittlere Flankenbreite beträgt 2,1 mm. Für die Strahlformung mit einer Subapertur (Abb. (5.14)b) ergibt sich FWHM = 4, $9 \mathrm{~mm}$ und eine mittlere Flankenbreite von 1,45 mm. Die Verringerung der Flankenbreite bei DPE Nr. IV im Vergleich zu DPE Nr. II ist eher ein marginaler Effekt. Die Strahlformung mit einer Subapertur zeigt in beiden Fällen die in Kapitel 3 diskutierten Intensitätsfluktuationen. Diese Fluktuationen überwiegen im Vergleich zur gezielten Variation der Intensität auf dem Träger des Spots durch den Deconvolutions-Ansatz im Design von DPE Nr. IV.

\subsubsection{Effizienzmessung von DPE Nr. IV}

Die Beugungseffizienz ist durch Pulsenergiemessungen mit einem Joulemeter bestimmt worden. In Abbildung (5.15) ist der entsprechende Aufbau skizziert. Die Messungen sind über 20 Pulse gemittelt worden.

Zum Vergleich der experimentellen Beugungseffizienz $\eta$ von DPE Nr. IV mit $\eta$ von DPE Nr. I-III wurde eine Kreisblende S mit $8 \mathrm{~mm}$ Durchmesser verwendet und zum Vergleich mit dem theoretischen Wert $\eta\left(\underline{\underline{I_{0}}}, \underline{\underline{I_{\mathrm{pc}}}}\right)$ (vgl. Tabelle (5.1)) für die Beugungseffizienz bei Verwendung partiell-kohärenter Strahlung eine Kreisblende mit $5 \mathrm{~mm}$ Durchmesser. In der folgenden Tabelle sind die Messwerte zusammengestellt.

\begin{tabular}{c|c|c|c} 
& M1 & M2 & Beugungseffizienz $\eta$ \\
\hline $\mathrm{S}(8 \mathrm{~mm})$ & $63,8 \mathrm{~mJ}$ & $44,2 \mathrm{~mJ}$ & $44,2 \mathrm{~mJ} / 63,8 \mathrm{~mJ}=69 \%$ \\
$\mathrm{~S}(5 \mathrm{~mm})$ & $63,9 \mathrm{~mJ}$ & $35,0 \mathrm{~mJ}$ & $35,0 \mathrm{~mJ} / 63,9 \mathrm{~mJ}=55 \%$
\end{tabular}




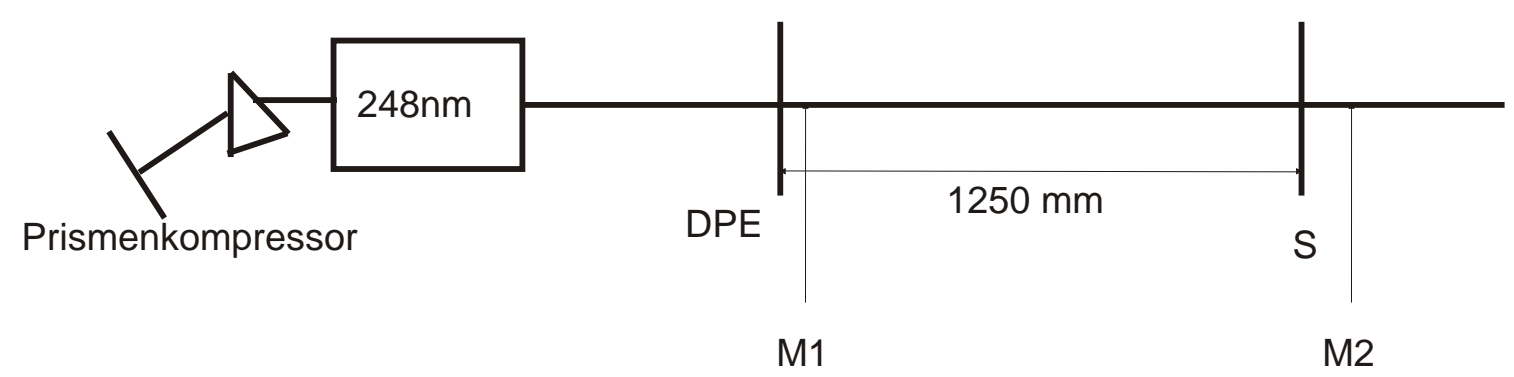

S: Kreisblende

M1/M2: Messung 1/2

$5 \mathrm{~mm}$ und $8 \mathrm{~mm}$ Durchmesser

Abbildung 5.15: Experimenteller Aufbau zur Effizienzmessung

Für $\eta(8 \mathrm{~mm})=69 \%$ ergibt sich eine gute Übereinstimmung mit dem Wert für DPE Nr. I von $71 \%$, ebenso für $\eta(5 \mathrm{~mm})=55 \%$ mit $\eta\left(\underline{\underline{I_{0}}}, \underline{\underline{I_{\mathrm{pc}}}}\right)=0,54$.

\subsection{Ergebnis}

Die zur optischen Rekonstruktion des DPE Nr. IV verwendete Excimer-Laserstrahlung zeigt eine ähnliche Wellenfrontverzerrung jedoch unterschiedliche Intensitätsverteilungen im Fernfeld im Vergleich zu den im Design von DPE Nr. IV berücksichtigten Strahleigenschaften. Das Konzept, die Wellenfrontverzerrung im Design eines DPE zu integrieren, hat sich auch in diesen Experimenten als sinnvoll erwiesen. Der Effekt der Korrektur der ortsaufgelösten Kohärenzeigenschaften durch den präsentierten Deconvolutions-Algorithmus ist jedoch geringer als die speckleartigen Intensitätsfluktuationen, die vom IFTA/IFQADesign verursacht werden. Auf die Verwendung der Ergebnisse des DeconvolutionsAlgorithmus in einem IFTA/IFQA-Design kann daher verzichtet werden. Geometrische Strahlformungskonzepte zum Design eines DPE (vgl. z.B. [16]) verursachen geringere Intensitätsfluktuationen [2] und bieten gegebenfalls die Möglichkeit, die Effekte des Deconvolutions-Algorithmus genauer zu studieren. 


\section{Zusammenfassung}

Innerhalb der vorliegenden Arbeit wurden neue Ansätze zur diffraktiven Strahlformung von partiell-kohärenter Laserstrahlung theoretisch entwickelt und experimentell überprüft.

Die für die kohärente Strahlformung entwickelte IFTA-Methode wurde übertragen auf die Berechnung der einzelnen Subaperturen diffraktiver Phasenelemente zur Formung partiell-kohärenter UV-Laserstrahlung. Aufgrund der geringen räumlichen Kohärenz der Strahlung kann die Überlagerung der Signale aus den einzelnen Subaperturen als inkohärent modelliert werden. Die Experimente zur optischen Rekonstruktion des so berechneten und hergestellten DPE Nr. I zeigten, dass die Qualität der Rekonstruktion im wesentlichen von zwei Faktoren abhängt. Erstens, die Strahlformung eines Teilstrahls, der auf eine Subapertur trifft, wird bestimmt durch den Kohärenzgrad der Strahlung. Mit abnehmender Kohärenz nehmen Intensitätsfluktuationen und Kantensteilheit der geformten Strahlprofile ebenfalls ab. Zweitens, wie passgenau sich die Signale aus den einzelnen Subaperturen überlagern, wird bestimmt durch die Wellenfrontverzerrung der verwendeten Strahlung.

Dieser Zusammenhang zwischen dem relativen Versatz der Signale und der Wellenfrontverzerrung konnte durch Shack-Hartmann-Messungen der Wellenfront verifiziert werden. Die gemessene Wellenfront wurde in den folgenden DPE Nr. II und Nr. III durch Korrekturterme berücksichtigt. Die optische Rekonstruktion dieser DPE zeigte eine passgenaue Überlagerung der einzelnen Signale. Die Kantensteilheit der überlagerten Rekonstruktion entspricht der Kantensteilheit der Rekonstruktion aus einer Subapertur, d.h. die Qualität der Rekonstruktion ist limitiert durch die räumliche Kohärenzlänge der Strahlung, die deutlich kleiner ist als die laterale Ausdehnung der einzelnen Subaperturen. Es sind somit erstmals die Ergebnisse von Wellenfrontmessungen in ein DPE zur Verbesserung der Strahlformung integriert worden. Mit dieser Technik ist es möglich, auf kollimierende Optiken im Strahlengang zu verzichten.

Weiterführend ist die Frage untersucht worden, inwiefern die Qualität der Strahlformung durch die Berücksichtigung der ortsaufgelösten Kohärenzeigenschaften verbessert werden kann. Es wurde eine neue Design-Methode entwickelt, wobei die Kohärenzfunktion $j$ der Strahlung für die einzelnen Subaperturen gemessen und durch einen zweistufigen Algorithmus zur Berechnung des DPE Nr. IV verwendet wurde. Die theoretische Simulation der Rekonstruktion des DPE Nr. IV ließ bei gleicher Beugungseffizienz eine Verbesserung der Homogenität des Profils von ca. 5\% (absolut) bzw. 27\% (relativ) erwarten.

Im Experiment konnte keine signifikante Verbesserung der Strahlformung im Vergleich 
zu DPE Nr. II festgestellt werden. Dies ist im wesentlichen auf die speckleartigen Intensitätsfluktuationen zurückzuführen, die vom IFTA/IFQA-Design verursacht werden.

Die ortsaufgelösten Kohärenzeigenschaften können daher bei DPE für die praktische Anwendung der Strahlformung von Excimer-Lasern vernachlässigt werden, denn die Methode des modifizierten IFTA-Designs mit Wellenfrontkorrektur liefert gute Ergebnisse bei deutlich geringerem Rechenaufwand. Über die Anwendungsaspekte hinaus ermöglichen die Ergebnisse der Arbeit ein tieferes Verständnis der Auswirkungen der partiellen Kohärenz in der Diffraktiven Optik. 


\section{A Konventionen und Definitionen}

Zeichen:

$$
\begin{aligned}
& \cdot \quad \text { skalare Multiplikation } \\
* & \text { Faltung } \\
f(x, y)= & g(\tilde{x}, \tilde{y}) * h(\tilde{x}, \tilde{y}) \\
= & \iint_{-\infty}^{+\infty} h(x-\tilde{x}, y-\tilde{y}) g(\tilde{x}, \tilde{y}) \mathrm{d} \tilde{x} \mathrm{~d} \tilde{y}
\end{aligned}
$$

Vektoren werden durch fettgedruckte Buchstaben dargestellt:

$$
\mathbf{v}=\left(\begin{array}{c}
v_{1} \\
\vdots \\
v_{n}
\end{array}\right) \in \mathbb{R}^{n}
$$

Das Skalarprodukt von Vektoren $\mathbf{v}, \mathbf{w} \in \mathbb{R}^{n}$ wird durch spitze Klammern gekennzeichnet:

$$
\langle\mathbf{v}, \mathbf{w}\rangle=\sum_{i}^{n} v_{i} w_{i}
$$

Matrizen durch doppelt unterstrichene Buchstaben:

$$
\underline{\underline{M}}=\left(\begin{array}{ccc}
m_{11} & \cdots & m_{1 m} \\
\vdots & \ddots & \vdots \\
m_{n 1} & \cdots & m_{n m}
\end{array}\right) \in \mathbb{R}^{n \times m}
$$

Kurzformen:

$$
\begin{aligned}
F T[f(u, v)](x, y) & =\int_{-\infty}^{\infty} \int_{-\infty}^{\infty} f(u, v) \exp [-2 \pi i(x u+y v)] \mathrm{d} u \mathrm{~d} v \\
\operatorname{rect}(\mathbf{x}, \Delta \mathbf{x}) & =\left\{\begin{array}{l}
1 \text { für }|x| \leq \Delta x / 2,|y| \leq \Delta y / 2 \\
0 \text { sonst }
\end{array}\right. \\
\operatorname{comb}(\mathbf{x}, \delta \mathbf{x}) & =\sum_{n, m=-\infty}^{\infty} \delta(x-n \delta x) \delta(y-m \delta y) \\
\operatorname{sinc}(\mathbf{x}, \delta \mathbf{x}) & =\frac{\sin (\pi x / \delta x)}{\pi x / \delta x} \frac{\sin (\pi y / \delta y)}{\pi y / \delta y}
\end{aligned}
$$


Die Wurzel des mittleren quadratischen Fehlers rms einer Matrix $\underline{\underline{A}} \in \mathbb{R}^{n \times m}$ bezüglich einer Matrix $\underline{\underline{S}} \in \mathbb{R}^{n \times m}$ ist definiert durch:

$$
r m s(\underline{\underline{S}}, \underline{\underline{A}}):=\sqrt{\frac{1}{N M} \sum_{n=1}^{N} \sum_{m=1}^{M}\left(S_{n m}-A_{n m}\right)^{2}} .
$$

Analog dazu der normierte $r m s$-Fehler für Matrizen mit $S_{n m}, A_{n m} \geq 0$ :

$$
r m s_{n}(\underline{\underline{S}}, \underline{\underline{A}}):=\frac{1}{\max (\underline{\underline{A}})} \sqrt{\frac{1}{N M} \sum_{n=1}^{N} \sum_{m=1}^{M}\left(S_{n m}-A_{n m}\right)^{2}} .
$$

Der skalierte $r m s$-Fehler wird benötigt, um Abweichungen durch unterschiedliche Skalierungen von $\underline{\underline{A}}$ und $\underline{\underline{S}}$ zu berücksichtigen:

$$
\begin{aligned}
r m s_{s}(\underline{\underline{S}}, \underline{\underline{A}}):=\sqrt{\frac{1}{N} \sum_{(n, m) \in \mathbb{S}}\left(S_{n m}-c_{s} \cdot A_{n m}\right)^{2}} \\
\mathbb{S}=\text { Indexmenge des Trägers von } \underline{\underline{S}} \\
N=\text { Anzahl der Pixel in } \mathbb{S}
\end{aligned}
$$

Der Skalierungsfaktor $c_{s}$ berechnet sich durch Nullsetzen der partiellen Ableitung von $r m s_{s}$ nach $c_{s}$ [47]. Dies führt auf den Ausdruck:

$$
c_{s}=\frac{\sum_{(n, m) \in \mathbb{S}} S_{n m} \cdot A_{n m}}{\sum_{(n, m) \in \mathbb{S}} A_{n m}^{2}} .
$$

Die Effizienz $\eta$ einer gegebenen Ist-Verteilung $\underline{\underline{A}}$ bezüglich einer Soll-Verteilung $\underline{\underline{S}}$ mit dem Träger $\mathbb{S}$ ist gegeben durch:

$$
\eta(\underline{\underline{S}}, \underline{\underline{A}}):=\frac{\sum_{(n, m) \in \mathbb{S}} A_{n m}}{\sum_{(n, m)} S_{n m}}
$$




\section{Abbildungsverzeichnis}

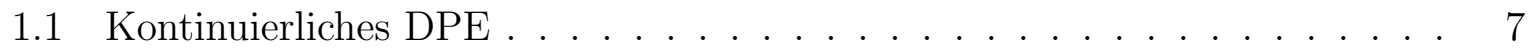

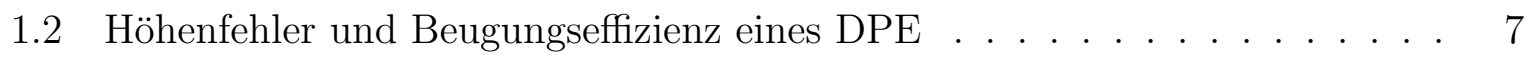

1.3 Quantisiertes DPE . . . . . . . . . . . . . . . . 8

1.4 IFTA-Schema . . . . . . . . . . . . . . . . . . . 9

1.5 IFQA-Schema . . . . . . . . . . . . . . . . . 11

1.6 Nah- und Fernfeld des L1000 Excimer-Lasers . . . . . . . . . . . . . . . 12

1.7 Prinzip der Homogenisierung durch Zylinderlisenarrays . . . . . . . . . 13

1.8 Herstellung eines mehrstufigen DPE . . . . . . . . . . . . . 15

1.9 REM Bilder des vierstufigen DPE Nr. I . . . . . . . . . . . . 16

2.1 Zur Notation bei Youngs Interferenz-Versuch . . . . . . . . . . . . . . 19

2.2 Prinzip des Shack-Hartmann Sensors _ . . . . . . . . . . . . . . 22

2.3 Shack-Hartmann Messung . . . . . . . . . . . . . . . . . . 23

3.1 DPE für die Mikromaterialbearbeitung . . . . . . . . . . . . 24

3.2 IFQA-Intensitätslösungen $\ldots \ldots \ldots \ldots \ldots \ldots \ldots \ldots \ldots \ldots$

3.3 Optische Rekonstruktion des DPE Nr. I mit HeNe-Laser $\ldots \ldots \ldots . . .28$

3.4 Experimenteller Aufbau DPE Nr. I . . . . . . . . . . . . . . . . . . . . 29

3.5 Optische Rekonstruktion des DPE Nr. I mit KrF Excimer-Laser . . . . . . 31

3.6 Räumlicher Versatz der Signale verschiedener Subaperturen . . . . . . . . 32

3.7 Experimenteller Aufbau zur Effizienzmessung, DPE Nr. I . . . . . . . . . . 33

4.1 Vorgebebene Intensität für DPE Nr. II/III . . . . . . . . . . . 36

4.2 Reproduzierbarkeit der Wellenfront . . . . . . . . . . . . . . 37

4.3 Experimenteller Aufbau DPE Nr. II/III . . . . . . . . . . . . . . . . 41

4.4 Wellenfront des Rekonstruktionsstrahls für DPE Nr. II/III . . . . . . . 42

4.5 Optische Rekonstruktion DPE Nr. II mit KrF Excimer-Laser . . . . . . . . 43

4.6 Optische Rekonstruktion DPE Nr. III mit KrF Excimer-Laser . . . . . . . 43

4.7 Experimenteller Aufbau zur Effizienzmessung, DPE Nr. II/III . . . . . . 44

4.8 Simulation der Beugungseffizienz in Abhängigkeit von Ätzfehlern für DPE Nr. II . . . . . . . . . . . . . . . . . . 44 46

4.9 Simulierte Itensitätsverteilung unter Berücksichtigung von Ätzfehlern . . . 47

4.10 Strukturfehler bei DPE Nr. II . . . . . . . . . . . . . . . . . 48

5.1 Experimenteller Aufbau zur ortsaufgelösten Fernfeldmessung . . . . . . . 50

5.2 Ortsaufgelöste Fernfeldmessungen des L1000 - Strahls mit Prismenkom-

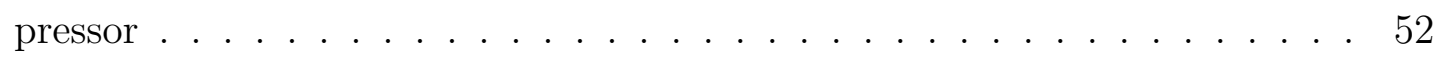


5.3 Ortsaufgelöste Fernfeldmessungen des L1000 - Rohstrahls . . . . . . . . . . 53

5.4 Separationsoperator $S e p O p \ldots \ldots \ldots \ldots$

5.5 TSVD-regularisierte Lösungen . . . . . . . . . . . . . . . . . . . 63

5.6 Simulierte Rekonstruktion eines idealen DPE mit partiell-kohärentem TSVD-Design . . . . . . . . . . . . . . . . . 6 64 64

5.7 Zu realisierende kohärente Intensitätsverteilung für das Design in Abb. $5.6 \quad 65$

$5.8 \mathrm{rms}_{\mathrm{s}}$-Fehler und Effizienz für TSVD-Regularisierung der Rohstrahlfern-

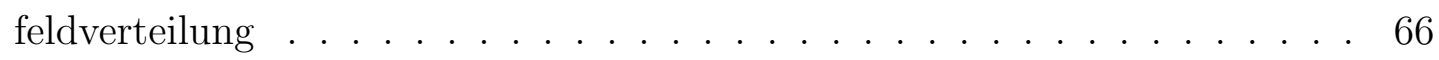

5.9 Simulierte Rekonstruktion eines idealen DPE mit partiell-kohärentem Design für den Rohstrahl . . . . . . . . . . . . . . . . . . . . . . . . . . . . . . 67

5.10 Tikhonov-regularisierte Lösungen . . . . . . . . . . . . . . . . . 68

5.11 Fernfeldmessungen des zur Rekonstruktion von DPE Nr. IV verwendeten L1000 Excimer-Laserstrahls . . . . . . . . . . . . . . . . . 72

5.12 Wellenfront für DPE Nr. IV: Design, Rekonstruktion, Differenz . . . . . . . 73

5.13 Optische Rekonstruktion DPE Nr. IV mit KrF Excimer-Laser . . . . . . . 74

5.14 Optische Rekonstruktion DPE Nr. II mit KrF Excimer-Laser . . . . . . . . 75

5.15 Experimenteller Aufbau zur Effizienzmessung, DPE Nr. IV . . . . . . . . 76 


\section{Tabellenverzeichnis}

1.1 Beugungseffizienz in Abhängigkeit der Stufenanzahl eines DPE . . . . . . 8

3.1 IFTA/IFQA-Werte für Objektfehler und Effizienz, DPE Nr. I . . . . . . . . 26

3.2 Gemessene Stufentiefen des DPE Nr. I . . . . . . . . . . . . . . . . 27

4.1 IFTA/IFQA-Werte für Objektfehler und Effizienz, DPE Nr. II/III . . . . . 39

4.2 Gemessene Stufentiefen der DPE Nr. II/III . . . . . . . . . . . . . . . . . 40

4.3 Ätztiefenfehler für DPE Nr. II/III . . . . . . . . . . . . . . . . . . . . 41

5.1 IFTA/IFQA-Werte für Objektfehler und Effizienz, DPE Nr. IV . . . . . . . 70 


\section{Literaturverzeichnis}

[1] Aagedal H., Schmid M., Beth T., Teiwes S. und Wyrowski F., Theory of speckles in diffractive optics and its application to beam shaping. J. mod. Opt., 7:1409-1421, 1996.

[2] Aagedal H., Wyrowski F. und Schmid M., Paraxial beam splitting and shaping. In: Turunen J. und Wyrowski F. (Herausgeber): Diffractive Optics For IndustriAL and Commercial Applications, Kapitel 6. Akademie Verlag Berlin, 1997.

[3] Allebach J. P., Gallagher N. C. und Liu B., Aliasing error in digital holography. Appl. Opt., 15:2183-2188, 1976.

[4] Basting D. (Herausgeber), Excimer Laser Technology: Laser Sources, Optics, Systems And Applications. Lambda Physik Göttingen, ISBN 3-00-006395-1, Göttingen, 2001.

[5] Bernges J., Unnebrink L. und Henning T., Mask adapted beam shaping for material processing with excimer laser radiation. SPIE, Vol. 3573:108-111, 1998.

[6] Bernhardt M., Wyrowski F. und Bryngdahl O., Iterative techniques to integrate different optical functions in a diffractive phase element. Appl. Opt., 30:4629-4635, 1991.

[7] Beyerlein M., Schuberth S. und Dresel T., Design and numerical analysis of diffractive optical elements for beam-shaping of partially coherent light. In: EOS TOPICAL Meeting Digest Series, Band 22, Seiten 157-158. (European Optical Society, Orsay, France), 1999.

[8] Born M. und Wolf E., Principles of Optics. Cambridge University Press, Cambridge, 7. Auflage, 1999.

[9] Bräuer R., Diffraktive Optik im Übergang zum Resonanzbereich. Dissertation, Universität Essen, 1994.

[10] Bräuer R., Wyrowski F. und Bryngdahl O., Diffusers in digital holography. J. Opt. Soc. Am. A, 8:572-578, 1991.

[11] Bryngdahl O., Geometrical transformations in optics. J. opt. Soc. Am ., 64:10921099, 1974. 
[12] Burghardt B. und Buecher H., Device for improving the beam quality of a laser. Patent Nr. DE4326191, 1995.

[13] Carter W. H. und Wolf E., Coherence and radiometry with quasihomogeneous planar sources. J. Opt. Soc. Am., 67:785-796, 1977.

[14] Dickey F. M. und O‘Neil B. D., Multifaceted laser beam integrators: general formulation and design concepts. Opt. Eng., 27:999-1007, 1988.

[15] Dresel T., Beyerlein M. und Schwider J., Design and fabrication of computergenerated beam-shaping holograms. Appl. Opt., 35:4615-4621, 1996.

[16] Dresel T., Beyerlein M. und Schwider J., Design of computer-generated beam-shaping holograms by iterative finite-element mesh adaption. Appl. Opt., 35:6865-6874, 1996.

[17] Ekberg M, Manufacture of high efficiency surface relief CGH. Technical Report No. 238, ISBN 91-7032-776-9, Chalmers University of Technology, Sweden, 1992.

[18] Friberg A. T. und Wolf E., Relationships between the complex degrees of coherence in the space-time and in the space-frequency domains. Opt. Lett., 20:623-625, 1995.

[19] Gabor D., A new microscopic principle. Nature, 161:777-778, 1948.

[20] Gale M. T. und Rossi M., Continuous-relief diffractive lenses and microlens arrays. In: Turunen J. und Wyrowski F. (Herausgeber): Diffractive Optics for InDustrial and Commercial Applications, Kapitel 4. Akademie Verlag Berlin, 1997.

[21] Golub G. H. und Van Loan C. F., Matrix Computations. John Hopkins University Press, Baltimore, 3. Auflage, 1996.

[22] Goodman J. W., Statistical Optics. John Wiley \& Sons, New York, 1985.

[23] Goodman J. W. und Silvestri A. M., Some effects of fourier-domain phase quantization. IBM J. Res. Develop., Seiten 478-484, 1970.

[24] Hansen P. C., Numerical Aspects of Deconvolution, Band 1 der Reihe Lecture Notes of the Department of Informatics and Mathematical Modelling of the Technical University of Denmark. Technical University of Denmark, Lyngby, Denmark, http://www.imm.dtu.dk/ pph/Regularization/deconv.html, 2000.

[25] Henning T. und Scholl M., Beamshaping by multifaceted integrator mirrors: effects of partial coherence. In: Weber H., Reng N., Lüdtke J. und Mejias P. M. (Herausgeber): Laser Beam Characterization, Seiten 117-128. Festkörper-Laser-Institut Berlin GmbH, Strasse des 17. Juni 135, 10623 Berlin, 1994. 
[26] Hentschel W. und Lauterborn W., Holographic Generation of Multi-Bubble Systems. In: Lauterborn W. (Herausgeber): CAVitation And Inhomogeneties IN UndeRWAter Acoustics, Springer Series in Electrophysics, Seiten 47-53. Springer Verlag Berlin, 1980.

[27] Holmer A.-K. und Hård S., Laser-machining experiment with an excimer laser and a kinoform. Appl. Opt., 34:7718-7723, 1995.

[28] Hopkins H. H., The concept of partial coherence in optics. Proc. Roy. Soc., A208:263-277, 1951.

[29] Jansson P. A., Deconvolution of Images And Spectra. Academic Press, San Diego, 2. Auflage, 1997.

[30] Johansson M. und Bengtsson J., Robust design method for highly efficient beamshaping diffractive optical elements using an iterative-Fourier-transform algorithm with soft operations. J. mod. Opt., 47:1385-1398, 2000.

[31] Kawata S., Hikima I., Ichihara Y. und Watanabe S., Spatial coherence of KrF excimer lasers. Appl. Opt., 31:387-396, 1992.

[32] Kley E.-B., Fuchs H.-J. und Zoellner K., A fabrication technique for high aspect ratio gratings. SPIE, 3879:71-75, 1999.

[33] Krackhardt U., Mait J. N. und Streibl N., Upper bound on the diffraction efficiency of phase-only fanout elements. Appl. Opt., 31:27-37, 1992.

[34] Krackhardt U., Streibl N. und Schwider J., Fabrication errors of computer generated multilevel phase-holograms. Optik, Seiten 137-146, 1994.

[35] Kress R., Linear Integral Equations. Springer-Verlag, New York, 2. Auflage, 1999.

[36] Kreutz E. W., Henning T., Bernges J., Unnebrink L., Poignand H., Poprawe R. und Loosen P., Intra- und Extra-Cavity-Strahlformung durch optische Komponenten mit variabler Reflexion. LaserOpto, 32(4):53-57, 2000.

[37] Lauterborn W., Kurz T. und Wiesenfeldt M., Coherent Optics. Springer Verlag, Berlin, 1995.

[38] Lee W.-H., Computer-Generated Holograms: Techniques and Applications. In: Wolf E. (Herausgeber): Progress in Optics, Band XVI, Seiten 121-232. Elsevier, Amsterdam, 1978. 
[39] Lesem L.B., Hirsch P. M. und Jordan J. A., The Kinoform: A new wavefront reconstruction device. IBM J. Res. Develop., Seiten 150-155, 1969.

[40] Mandel L. und Wolf E., Optical Coherence and Quantum Optics. Cambridge University Press, Cambridge, 1995.

[41] Mann K., Hopfmüller A., Gerhardt H., Gorzellik P., Schild R., Stöffler W., Wagner H. und Wolbold G., Monitoring and shaping of excimer laser beam profiles. Laser und Optoelektronik, 24(1):43-49, 1992.

[42] Markis II R. J., Introduction to Shannon Sampling and Interpolation TheORY. Springer Verlag, New York, 1991.

[43] Nikolajeff F., Hård S. und Curtis B., Diffractive microlenses replicated in fused silica for excimer laser-beam homogenizing. Appl. Opt., 36:8481-8489, 1997.

[44] Römer H., Theoretische Optik. VCH, Weinheim, 1994.

[45] Schäfer B. und Mann K., Laserstrahlcharakterisierung mit dem Hartmann-ShackWellenfrontsensor. LaserOpto, 32(6):47-52, 2000.

[46] Schäfer B. und Mann K., Investigation of the propagation characteristics of excimer lasers using a Hartmann-Shack sensor. Rev. Sci. Instrum., 71:2663-2668, 2000.

[47] Seldowitz M. A., Allebach J. P. und Sweeney D. W., Synthesis of digital holograms by direct binary search. Appl. Opt., 26:2788-2798, 1987.

[48] The MathWorks, Inc., MatLab Reference Guide. The MathWorks, Inc., Natick, MA 01760-1500, USA, 1996.

[49] Turunen J., Pääkkönen P., Kuittinen M., Laakkonen P., Simonen J., Kajava T. und Kaivola M., Diffractive shaping of excimer laser beams. J. mod. Opt., 47:2467-2475, 2000.

[50] Turunen J. und Wyrowski F. (Herausgeber), Diffractive Optics for Industrial and Commercial Applications. Akademie Verlag Berlin, 1. Auflage, 1997.

[51] Vahimaa P., Studies on diffractive optics and partial coherence. Dissertation, University of Joensuu, Finland, 1996.

[52] Williams S.W., Marsden P. J., Roberts N. C., Sidhu J. und Venables M. A., Excimer laser beam shaping and material processing using diffractive optics. SPIE, Vol. 3343:205-211, 1998. 
[53] Wyrowski F., Considerations on convolutions and phase factors. Opt. Com., 81:353358, 1990.

[54] Wyrowski F., Diffractive optical elements: iterative calculation of quantized, blazed phase structures. J. Opt. Soc. Am. A, 7:961-969, 1990.

[55] Wyrowski F., Upper bound of the diffraction efficiency of diffractive phase elements. Opt. Lett., 24:1915-1917, 1991.

[56] Wyrowski F. und Bryngdahl O., Digital holography as part of diffractive optics. Rep. Prog. Phys., Seiten 1481-1571, 1991. 


\section{Veröffentlichungen:}

D. Schäfer

Design concept for diffractive elements shaping partial coherent laser beams

J. Opt. Soc. Am. A, November 2001, zur Veröffentlichung akzeptiert.

$\square$ P.R. Herman, K.P. Chen, M. Wei, J. Zhang, J. Ihlemann, D. Schäfer, G. Marowsky, P. Oesterlin, B. Burghardt $F_{2}$-lasers: High resolution optical processing system for shaping photonic components SPIE 4274 , im Druck.

D. Schäfer, J. Ihlemann, G. Marowsky, P.R. Herman $F_{2}$-laser ablation patterning of dielectric layers Appl. Phys. A $\underline{72}$, 377-379, 2001.

$\square$ D. Schäfer, J. Ihlemann, G. Marowsky, B. Burghardt, M. Timm Multifacet kinoforms for KrF excimer laser Diffractive Optics and Micro-Optics, OSA Technical Digest, pp. 189-191, 2000.

D. Schäfer, H. Goenner The gravitational field of a radiating and contracting spherically-symmetric body with heat flow General Relativity and Gravitation, 32, 2119-2130, 2000.

D. Schäfer, G. Marowsky Laserlicht auf neuen Wegen Diffraktive Elemente formen Laserstrahlen in beliebige Profile Neue Zürcher Zeitung, p. 55, 22. März 2000.

$\square$ D. Schäfer, J. Ihlemann, K. Mann, G. Marowsky Excimer laser patterned dielectric masks for the fabrication of diffractive optical elements by laser ablation Appl. Phys. A 69, S319-S322, 1999.

D. Schäfer, J. Ihlemann, K. Rubahn, K. Mann, G. Marowsky Fabrication of diffractive optical elements using dielectric masks EOS Topical Meetings Digest Series Vol. 22, 48-49, 1999.

D. Schäfer, J. Ihlemann, K. Mann, G. Marowsky Fabrication of diffractive optical elements by laser ablation using excimer laser patterned dielectric masks $5^{\text {th }}$ International Conference on Laser Ablation - COLA 99, Göttingen, 19.-23.7.1999. 


\section{Vielen Dank an ...}

Herrn Prof. Dr. W. Lauterborn und Herrn Prof. Dr. G. Marowsky für die Betreuung dieser Arbeit,

Dr. H.-J. Kahlert, Dr. B. Burghardt, Dr. P. Oesterlin, Frank Simon, Rolf Senczuk und Andre Kolanek für die Unterstützung bei den Experimenten und für die Diskussionen über die Ergebnisse,

Bernd Schäfer für die Hilfe bei den Wellenfrontmessungen und für die Diskussionen über die Konzepte der partiellen Kohärenz,

meine Arbeitsgruppe Jürgen Ihlemenn, Martin Heßling, Stefan Puschmann, Sabine Müller und Ruth Weichenhain für das angenehme Arbeitsklima. Insbesondere Dank an Jürgen, der mich in die Geheimnisse des experimentellen Arbeitens und des Paper-Schreibens einführte,

Uwe Bünting für die Hilfe beim Programmieren und die gemeinsame Zeit im Büro,

Martin, Sabine, Stefan und Jürgen für das Korrekturlesen,

meine treue und nachsichtige Mensagruppe und

Susanne für ihre liebevolle Unterstützung in allen Lebenslagen und Phasen der Promotion. 


\title{
Lebenslauf:
}

\author{
Dirk Schäfer \\ 1972 \\ geboren in Bremen \\ 8/78 Einschulung in Bremen \\ 6/91 Abitur in Bremen \\ $8 / 91-10 / 92$ \\ Zivildienst in Bremen \\ $10 / 92-10 / 93$ \\ Grundstudium Physik an der Universität Bremen \\ 10/93-10/94 \\ Grundstudium Physik an der Universität Göttingen \\ 10/94 Vordiplom Physik an der Universität Göttingen \\ $10 / 94-7 / 98$ \\ Hauptstudium Physik an der Universität Göttingen \\ 4/95-7/95 \\ Studienaufenthalt bei Prof. Marest am Institut Physique Nucléaire, \\ Université de Lyon, France. \\ $1 / 97-7 / 98$ \\ Diplomarbeit am Institut für Theoretische Physik bei Prof. \\ Goenner. Thema: „Das Gravitationsfeld relativistischer, zentral- \\ symmetrischer Sternmodelle mit wärmeleitender Materie und \\ Abstrahlung". \\ 7/98 Diplom in Physik an der Universität Göttingen \\ seit $10 / 98$ \\ Wissenschaftlicher Mitarbeiter am Laser-Laboratorium Göttingen \\ e.V. und Beginn der Dissertation unter Betreuung von Prof. Lau- \\ terborn und Anleitung von Prof. Marowsky.
}

